\title{
División de Ciencias Básicas e Ingeniería
}

\author{
"ESTUDIO TEÓRICO DE LA REDUCCIÓN \\ DEL $\mathrm{N}_{2} \mathrm{O}$ SOBRE NANOPARTÍCULAS DE \\ $\mathrm{PT}_{8} \mathrm{Y} \mathrm{PT}_{8} \mathrm{O}$, EMPLEANDO TEORÍA DE \\ FUNCIONALES DE LA DENSIDAD (DFT)".
}

Tesis que presenta

Erendida Hernández Vera

Para obtener el grado Académico de

Maestro en Ciencias (Química)

Asesora: Dra. Virineya Sonia Bertin Mardel

Jurado Calificador:

Presidente: $\quad$ Francisco Miguel Castro Martínez

Secretario: Marco Antonio Mora Delgado

Vocal: $\quad$ Annia Galano Jiménez

México, D.F. Noviembre del 2014 

Dedicada a

mis padres con cariña:

Canrada \#ernández Zaragoza

y Adela Vera Vera 


\section{Agradezca}

- A la asesara de este proyecta Dra. Virineya Bertín Mardel, par su tiempa, dedicación y enseñanza para realizar y culminar can éxita este proyecta.

- A mis sinadales: Dra. Annia Galana Diménez. Dr. Marca Antania Mora Delgada y al Dr. Francisca Miguel Castra Martinez par el tiempa dedicada a evaluar mi trabaja de maestría.

- A la universidad Autónoma Metropalitana-Unidad-Iztapalapa (UAM- 1), par el apoya y facilidad para realizar can éxita mi tesis de maestria y par ser mi casa de estudias. 
Agradezca

a todos mis amigos agradezca su apoyo, paciencia y amistad. . . 


\section{Tabla de contenido}

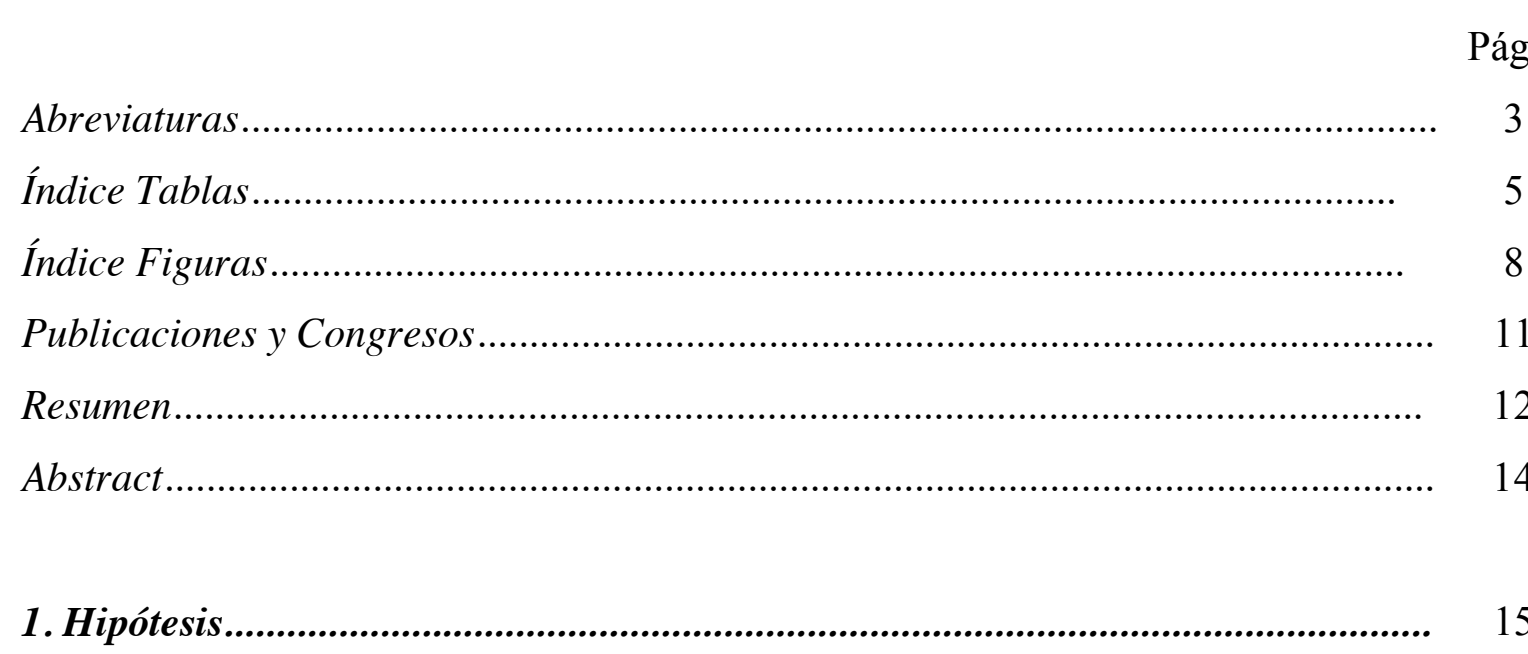

Página

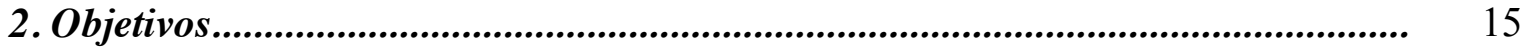

2.1 Objetivo general. 15

2.2 Objetivos específicos. 15

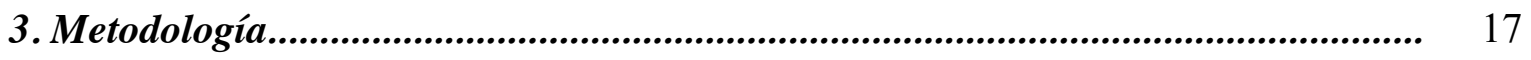

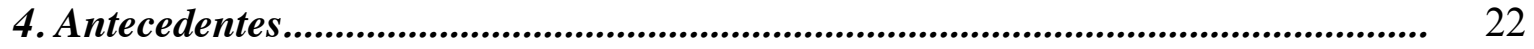

4.1 El contaminante $\mathrm{N}_{2} \mathrm{O}$. 22

4.2 Descripción de las reacciones en los dispositivos catalíticos. 24

$\begin{array}{ll}4.3 \text { Estudios teóricos sobre nanopartículas de Pt. } & 27\end{array}$

5. Resultados y discusión.......................................................................... 31

5.1 Propiedades químicas de platino $\mathrm{Pt}_{2} \mathrm{y} \mathrm{N}_{2} \mathrm{O}$. 31

5.2 Reacción de disociación del $\mathrm{N}_{2} \mathrm{O}$ catalizada por nanopartículas de $\mathrm{Pt}_{8}$. 34

5.2.1 Optimización geométrica de las nanopartículas de $\mathrm{Pt}_{8}$. Estructuras geométricas y energéticas. 35

5.2.2 Estudio de la disociación del $\mathrm{N}_{2} \mathrm{O}$ sobre cúmulos de $\mathrm{Pt}_{8}$. 41 Identificación de los sitios de activación por $\mathrm{N}_{2} \mathrm{O}$. 
5.2.3 Análisis de cargas Hirshfeld de la reacción de $\mathrm{N}_{2} \mathrm{O}$ sobre $\mathrm{Pt}_{8}$.

5.3 Reacción de disociación del $\mathrm{N}_{2} \mathrm{O}$ catalizada por nanopartículas oxidadas 58 de platino $\mathrm{Pt}_{8} \mathrm{O}$.

5.3.1 Optimización geométrica de la nanopartícula $\mathrm{Pt}_{8} \mathrm{O}$. 58

5.3.2 Estudio de la disociación del $\mathrm{N}_{2} \mathrm{O}$ sobre la nanopartícula de 67

$\mathrm{Pt}_{8} \mathrm{O}$. Identificación de los sitios de activación por $\mathrm{N}_{2} \mathrm{O}$.

5.3.3 Análisis de cargas Hirshfeld de la reacción de $\mathrm{N}_{2} \mathrm{O}$ sobre $\mathrm{Pt}_{8}$.

6. Conclusiones.

7. Perspectivas.

8. Bibliografía.

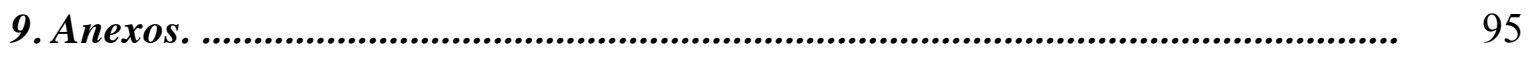

9.1.1 ADF: Ámsterdam Density Funtional Software 95

9.1.2 Efectos Relativistas

$\begin{array}{ll}\text { 9.1.3 Cargas atómicas } & 102\end{array}$

9.1.4 Presentación resumida de la Teoría de Funcionales de la Densidad 104

9.1.5 Continuación resultados: Reacción $\mathrm{N}_{2} \mathrm{O}+\mathrm{Pt}_{8} \quad 107$

9.1.6 Continuación resultados: Reacción $\mathrm{N}_{2} \mathrm{O}+\mathrm{Pt}_{8} \mathrm{O}$ 


\section{Abreviaturas}

\begin{tabular}{|c|c|}
\hline Símbolo & Significado \\
\hline$\AA$ & Angstroms, unidad de distancia equivale $1 \AA=1 \times 10^{-10} \mathrm{~m}=0,1 \mathrm{~nm}$. \\
\hline $\mathrm{ADF}$ & Programa de cálculo DFT (Ámsterdam Density Funtional) \\
\hline $\mathrm{Al}_{2} \mathrm{O}_{3}$ & Alúmina \\
\hline $\mathrm{BE}$ & Energía de enlace \\
\hline $\mathrm{CaO}$ & Óxido de calcio \\
\hline $\mathrm{CH}_{4}$ & Metano \\
\hline $\mathrm{CO}$ & Monóxido de carbono \\
\hline $\mathrm{CO}_{2}$ & Dióxido de carbono \\
\hline $\mathrm{CuO}$ & Óxido de cobre \\
\hline $\mathrm{Cu} / \mathrm{ZSM}-5$ & Cobre soportado en zeolita (Zeolita Socony Mobil - 5) \\
\hline DFT & Teoría de las funcionales de la densidad \\
\hline$d_{N-N}$ & Distancia nitrógeno-nitrógeno \\
\hline$d_{\mathrm{N}-\mathrm{O}}$ & Distancia nitrógeno-oxígeno \\
\hline $\mathrm{d}_{\mathrm{Pt}-\mathrm{N}}$ & Distancia platino-nitrógeno \\
\hline $\mathrm{d}_{\mathrm{Pt}-\mathrm{O}}$ & Distancia platino-oxígeno \\
\hline$\AA_{\mathrm{Pt}-\mathrm{Pt}}$ & Distancia promedio platino-platino \\
\hline $\pm đ_{\mathrm{Pt}-\mathrm{Pt}}$ & Desviación estándar de la distancia promedio platino-platino \\
\hline$E_{\text {ads }}$ & Energía de adsorción \\
\hline $\mathrm{E}_{\mathrm{dis}}$ & Energía de disociación \\
\hline $\mathrm{E}_{\text {relativa }}$ & Energía relativa \\
\hline GGA & Aproximación de Gradiente Generalizado \\
\hline GTO & Orbitales Tipo Gausianas \\
\hline HFC & Hidrofluorocarbono \\
\hline $\mathrm{Lu}_{2} \mathrm{O}_{3}$ & Óxido de lutecia \\
\hline $\mathrm{M}$ & Multiplicidad de espín \\
\hline $\mathrm{Mg}$ & Magnesio \\
\hline
\end{tabular}




\begin{tabular}{|c|c|}
\hline $\mathrm{MgO}$ & Óxido de magnesio \\
\hline $\mathrm{NO}$ & Monóxido de nitrógeno \\
\hline $\mathrm{N}_{2} \mathrm{O}$ & Dióxido de nitrógeno \\
\hline $\mathrm{NO}_{2}$ & Óxido nitroso \\
\hline $\mathrm{NO}_{\mathrm{x}}$ & Óxidos de nitrógeno \\
\hline PBE & Perdew-Burke-Ernzerhof \\
\hline $\mathrm{PFC}$ & Perfluorocarbono ó fluorocarbonos \\
\hline $\mathrm{ppb}$ & Partes por billón \\
\hline $\mathrm{Pt}_{8}$ & Cúmulo de ocho átomos de platino \\
\hline $\mathrm{Pt}_{\mathrm{n}}$ & Cúmulo de n-átomos de platino \\
\hline $\mathrm{Pt}_{8} \mathrm{O}$ & Cúmulo oxidado de 8 átomos de platino \\
\hline $\mathrm{q}_{\mathrm{i}}$ & Carga inicial \\
\hline $\mathrm{q}_{\mathrm{f}}$ & Carga final \\
\hline$\Delta \mathrm{q}$ & Carga final-carga inicial \\
\hline $\mathrm{Rh}_{5}^{+}$ & Catión rodio de cinco átomos \\
\hline $\mathrm{SF}_{6}$ & Hexafluoruro de azufre \\
\hline $\mathrm{SO}_{2}$ & Dióxido de azufre \\
\hline STO & Orbital Tipo Slater \\
\hline TBE & Energía Total de Enlace \\
\hline $\mathrm{TZ2P}$ & Triple Zeta doble Polarización \\
\hline$v$ & Frecuencia de vibración \\
\hline $\mathrm{v}_{\text {calc }}$ & Frecuencia de vibración calculada \\
\hline$U_{\exp }$ & Frecuencia de vibración experimental \\
\hline ZORA & Aproximación Regular de Orden Cero \\
\hline
\end{tabular}




\section{Índice Tablas}

Pág.

Tabla 5.1.1 Parámetros principales de Pt $t_{2}$ calculados. Energías de enlace (BE), $\quad 31$ energías relativas $\left(E_{\text {relativa }}\right)$, frecuencias de vibración $(v)$ y distancias de enlace $\left(d_{P t-P t}\right)$.

Tabla 5.1.2 Comparación de resultados teóricos y experimentales para Pt $t_{2} .32$ Energía de enlace (BE )en kcal/mol.

Tabla 5.1.3 Principales parámetros de la molécula de $\mathrm{N}_{2} \mathrm{O}$. Distancias de enlace

$\left(d_{N-N}, d_{N-O}\right)$, frecuencias de vibración (v) y cargas Hirshfeld, calculados y experimentales.

Tabla 5.2.1.1 Parámetros energéticos de estructuras geométricas de Pts. Multiplicidad de espín (M), energías de enlace (BE) y energía relativa $\left(E_{\text {relativa }}\right)$ de los seis isómeros de platino.

Tabla 5.2.1.2 Parámetros geométricos de estructuras optimizadas de Pts. Multiplicidad de espín (M), frecuencias de vibración ( $v$ ) y distancias de enlace $(d)$.

Tabla 5.2.2.1 Parámetros energéticos de la interacción de $\mathrm{N}_{2} \mathrm{O}$ hacia átomos del isómero $I$. Energía de disociación $\left(E_{\text {dis }}\right)$ y energía relativa $\left(E_{\text {relativa}}\right)$ respecto al de más baja energía.

Tabla 5.2.2.2 Parámetros geométricos de la interacción de $\mathrm{N}_{2} \mathrm{O}$ hacia átomos del isómero I. Multiplicidad de espín (M), frecuencias de vibración (v) y distancias de enlace $(d)$.

Tabla 5.2.2.3 Parámetros de energía, interacción de $\mathrm{N}_{2} \mathrm{O}$ hacia enlaces del 50 isómero I. Energías de disociación $\left(E_{\text {dis }}\right)$ y energía relativa $\left(E_{\text {relativa }}\right)$.

Tabla 5.2.2.4 Parámetros geométricos, interacción de $\mathrm{N}_{2} \mathrm{O}$ hacia enlaces del isómero I. Multiplicidad de espín (M), frecuencias de vibración (v) y distancias de enlace $(d)$. 
Tabla 5.2.2.5 Parámetros de energía, interacción de $\mathrm{N}_{2} \mathrm{O}$ hacia caras del isómero I. 119 Energías de disociación $\left(E_{\text {dis }}\right)$ y energía relativa $\left(E_{\text {relativa }}\right)$.

Tabla 5.2.2.6 Parámetros geométricos interacción de $\mathrm{N}_{2} \mathrm{O}$ hacia caras del isómero I. Multiplicidad (M), Frecuencias de vibración (v) y distancias de enlace $(d)$.

Tabla 5.2.3.1 Análisis de cargas Hirshfeld de las interacciones del $\mathrm{N}_{2} \mathrm{O}$ hacia el 55 isómero I, con acercamientos por átomos. Cargas iníciales $\left(q_{i}\right)$, cargas finales $\left(q_{f}\right)$ y cambio total de carga $\left(\Delta q=q_{f}-q_{i}\right)$ para todos los átomos.

Tabla 5.2.3.2 Análisis de cargas Hirshfeld de las interacciones del $\mathrm{N}_{2} \mathrm{O}$ hacia el 57 isómero I, con acercamientos por enlaces. Cargas iníciales $\left(q_{i}\right)$, cargas finales $\left(q_{f}\right)$ y cambio total de carga $\left(\Delta q=q_{f}-q_{i}\right)$ para todos los átomos.

Tabla 5.3.1.1 Parámetros energéticos de estructuras optimizadas de $\mathrm{Pt}_{8} \mathrm{O}$ con 60 adsorción de $\mathrm{O}$ en átomos del isómero I. Energías de adsorción $\left(E_{a d s}\right)$ y energía relativa $\left(E_{\text {relativa }}\right)$.

Tabla 5.3.1.2 Parámetros geométricos para estructuras optimizadas de $\mathrm{Pt}_{8} \mathrm{O}$ con 61 adsorción de $\mathrm{O}$ en átomos del isómero I. Multiplicidad de espin (M), frecuencias de vibración (v), distancias de enlace $(d)$.

Tabla 5.3.1.3 Parámetros energéticos de estructuras optimizadas de $\mathrm{Pt}_{8} \mathrm{O}$ con adsorción del átomo de $O$ en enlaces Pt-Pt del isómero I. Energías de adsorción $\left(E_{a d s}\right)$ y energía relativa $\left(E_{\text {relativa }}\right)$.

Tabla 5.3.1.4 Parámetros geométricos de estructuras optimizadas de $\mathrm{Pt}_{8} \mathrm{O}$ con adsorción de $\mathrm{O}$ en enlaces Pt-Pt del isómero I. Multiplicidad (M), frecuencias de vibración (v) y distancia de enlace (d).

Tabla 5.3.1.5 Parámetros energéticos de estructuras de $\mathrm{Pt}_{8} \mathrm{O}$ con adsorción de $\mathrm{O}$ en 66 caras del isómero I. Energías de adsorción $\left(E_{a d s}\right)$ y energía relativa $\left(E_{\text {relativa }}\right)$.

Tabla 5.3.1.6 Parámetros geométricos de estructuras de $\mathrm{Pt}_{8} \mathrm{O}$ con adsorción del 66 átomo de $O$ en caras del isómero I. Multiplicidad de espín (M), frecuencias de vibración (v) y distancias de enlace (d). 
Tabla 5.3.2.1 Parámetros energéticos de la interacción del $\mathrm{N}_{2} \mathrm{O}$ sobre átomos de Pt

del isómero oxidado, IO-E25, por el átomo de O del óxido. Energías de disociación $\left(E_{\text {dis }}\right)$ y energía relativa $\left(E_{\text {relativa }}\right)$.

Tabla 5.3.2.2 Parámetros geométricos de la interacción de $\mathrm{N}_{2} \mathrm{O}$ sobre átomos de Pt del isómero oxidado, IO-E25, por el átomo de $O$ del óxido. Multiplicidad de espín (M), frecuencias de vibración (v), distancias de enlace $(d)$.

Tabla 5.3.2.3 Parámetros energéticos de la interacción de $\mathrm{N}_{2} \mathrm{O}$ sobre átomos del 75 isómero oxidado, IO-E25, por el átomo de $N$ terminal. Energías de disociación $\left(E_{\text {dis }}\right)$ y energía relativa $\left(E_{\text {relativa }}\right)$.

Tabla 5.3.2.4 Parámetros geométricos de la interacción de $\mathrm{N}_{2} \mathrm{O}$ sobre átomos del 76 isómero oxidado, IO-E25, por el átomo de $N$ terminal (N1). Multiplicidad (M), Frecuencias de vibración (v) y distancias de enlace $(d)$.

Tabla 5.3.2.5 Parámetros energéticos de la interacción de $\mathrm{N}_{2} \mathrm{O}$ en enlaces Pt-Pt del 79 isómero oxidado, IO-E25. Energías de disociación $\left(E_{\text {dis }}\right)$ y energía relativa $\left(E_{\text {relativa }}\right)$.

Tabla 5.3.2.6 Parámetros geométricos para la interacción de $\mathrm{N}_{2} \mathrm{O}$ en enlaces Pt-Pt, del isómero oxidado, IO-E25. Multiplicidad (M), frecuencias de vibración (v), distancias de enlace $(d)$.

Tabla 5.3.3.1 Análisis de cargas Hirshfeld de las interacciones del $\mathrm{N}_{2} \mathrm{O}$ hacia el isómero oxidado, IO-E25, con acercamientos hacia átomos por el lado del $O$ del oxido. Cargas iníciales $\left(q_{i}\right)$, finales $\left(q_{f}\right)$ y cambio total de carga $\left(\Delta q=q_{f}-q_{i}\right)$ para todos los átomos.

Tabla 5.3.3.2 Análisis de cargas Hirshfeld de las interacciones del $\mathrm{N}_{2} \mathrm{O}$ hacia el 84 isómero oxidado, IO-E25, con acercamientos hacia enlaces. Cargas iníciales $\left(q_{i}\right)$, finales $\left(q_{f}\right)$ y cambio total de carga $\left(\Delta q=q_{f}-q_{i}\right)$ para todos los átomos. 


\section{Índice Figuras}

Pág.

Figura 4.1.1 Emisiones de $\mathrm{N}_{2} \mathrm{O}$ en U.S. Fuente: Inventory of U.S. Greenhouse 22 Gas Emissions and Sinks: 1990-2012.

Figura 4.2.1 Evolución del precio de los metales de transición usados en los 26 convertidores catalíticos: Rodio, Platino, Paladio y Oro. versus Precio en dólares (US)/onza Troy.

Figura 5.1.1 Geometría optimizada de la molécula de $\mathrm{N}_{2} \mathrm{O}$ (singulete).

Figura 5.2.1.1 Estructuras optimizadas de isómeros de Pt8 más estables con M 36 correspondiente.

Figura 5.2.1.2 Distancias de enlace y diferentes vistas de geometría del isómero I: a) vista desde arriba, $b$ ) vista lateral izquierda, c) vista frontal yd) vista desde atrás.

Figura 5.2.1.3 a) Plano de simetría formado por los átomos 1, 2, 4 y 8, divide 40 geometría de la nanopartícula de ${ }^{3} \mathrm{Pt}_{8}$ (Isómero I) en dos partes iguales. b) Distribución de cargas Hirshfeld de la nanopartícula $d e^{3} \mathrm{Pt}_{8}$ (Isómero I).

Figura 5.2.2.1 Aproximaciones de la molécula de $\mathrm{N}_{2} \mathrm{O}$ hacia el isómero I, a) 41 lineal al átomo de Pt por el oxígeno, $b$ ) lineal por el $N$ terminal $y$ c) el $N$ intermedio al átomo de Pt, perpendicular al eje de simetría de Pt.

Figura5.2.2.2 Geometrías iníciales de interacción del $\mathrm{N}_{2} \mathrm{O}$ hacia isómero I, a) 42 I-A8O por el átomo de $O$ y b) I-A8N1 por el átomo de $N$ terminal, con diferentes multiplicidades de espín $(M=2 s+1) .(*)$ No hay resultados, problemas de convergencia con el cálculo.

Figura 5.2.2.3 Acercamientos del $\mathrm{N}_{2} \mathrm{O}$ hacia el isómero $\mathrm{I}$, a) paralelo a dos átomos de $P t, b)$ perpendicular al enlace de Pt por el átomo de $O, c)$ perpendicular al enlace de Pt por el átomo de $N$ terminal $y$ d) perpendicular al enlace de Pt por el átomo de $N$ intermedio. 
Figura 5.2.2.4 Geometrías iníciales de interacción $\mathrm{Pt}_{8}+\mathrm{N}_{2} \mathrm{O}$ en diferentes

enlaces Pt-Pt del isómero I y sus resultados con distintas multiplicidades de espín, $M=2 s+1$. (**) interacciones en sitios equivalentes al indicado, por simetría del isómero $I$.

Figura 5.2.2.5 Acercamientos de $\mathrm{N}_{2} \mathrm{O}$ hacia una cara del isómero I de Pt, a) 52 perpendicular por el átomo de $N, b)$ perpendicular por el átomo de $O, c)$ paralelo e interaccionando el átomo de $O$ con un átomo de $\mathrm{Pt}$ del vértice, d) paralelo e, interaccionando el átomo de $\mathrm{N}$ terminal con un átomo de Pt del vértice, e) paralelo a una cara, interaccionando con dos vértices de la cara.

Figura 5.2.2.6 Geometrías iníciales de interacción $\mathrm{Pt}_{8}+\mathrm{N}_{2} \mathrm{O}$ en caras del isómero Iy sus resultados con distintas multiplicidades de espín, $M=2 s+1$. (**) Interacciones en sitios equivalentes al indicado, por simetría del isómero Ise generan estos sitios.

Figura 5.2.3.1 Acercamientos favorables a la disociación de la molécula del oxido. El sitio más reactivos esta en el átomo 8 en ambas interacciones, a) ${ }^{3}$ I-A8N1 y b) ${ }^{5}$ I-A8O.

Figura 5.2.3.2 Acercamientos favorables a la disociación de la molécula del 56 óxido. Los sitos reactivos son los siguientes: a) I-E58, b) I-E57, c) I-E25, d) I-E27, e) I-E14. Todos los acercamientos a enlaces son paralelos a el eje de la molécula.

Figura 5.3.1.1 Geometrías estables para la adsorción del átomo de oxígeno sobre el isómero I, en diferentes sitios de esta. Con multiplicidades de espín, $M=2 s+1$. (**Adsorciones del átomo de $O$ en posiciones equivalentes al que se adsorbe, por simetría del isómero I sin oxidar se generan estos sitios equivalentes).

Figura 5.3.1.2 Geometrías más estables para la adsorción del átomo de oxígeno sobre el isómero I en diferentes sitios formados por enlaces en este, con sus multiplicidades de espín, $M=2 s+1$. (**Adsorciones del átomo de $\mathrm{O}$ en posiciones equivalentes al que se adsorbe, por simetría del isómero I sin oxidar se generan estos sitios equivalentes). 
Figura 5.3.1.3 Geometrías más estables para la adsorción de un átomo de oxígeno sobre algunas caras del isómero I de Pt, con sus multiplicidades de espín, $M=2 s+1$. (**Adsorciones del átomo de $O$ en posiciones equivalentes al que se adsorbe, por la simetría del isómero Isin oxidar se generan estos sitios equivalentes).

Figura 5.3.2.1 a) Estructura geométrica de mínima energía para el isómero I 68 oxidado, ${ }^{1} I O-E 25$ ( ${ }^{1}$ IO-E23), b) distribución de carga Hirshfeld en el isómero oxidado (zonas rojas ricas en e- y zonas azules deficientes). **Sitios de oxidación equivalentes al indicado, por simetría inicial del isómero Isin oxidar.

Figura 5.3.2.2 Resultados de interacción de $\mathrm{Pt}_{8} \mathrm{O}+\mathrm{N}_{2} \mathrm{O}$ en diferentes átomos Pt por el lado del $O$, con distintas multiplicidades de espín $(M=2 s+1)$. (*) Interacciones con problemas de convergencia, no se muestran resultados.

Figura 5.3.2.3 Resultados de interacción de $\mathrm{Pt}_{8} \mathrm{O}+\mathrm{N}_{2} \mathrm{O}$ en diferentes átomos Pt del isómero I oxidado, IO-E25, por el lado del $N$ terminal, con distintas multiplicidades de espín $(M=2 s+1)$.

Figura 5.3.2.4 Resultados de interacción de Pt ${ }_{8} \mathrm{O}+\mathrm{N}_{2} \mathrm{O}$ en diferentes enlaces Pt-Pt del isómero I oxidado, IO-E25, con distintas multiplicidades de espín 1, 3, 5 y $7(M=2 s+1)$.

Figura 5.3.2.5 Interacciones de $\mathrm{N}_{2} \mathrm{O}$ con resultados favorables de disociación. Acercamientos directos por el $\mathrm{O}$ del óxido hacia un átomo de Pt.

Figura 5.3.2.6 Interacciones de $\mathrm{N}_{2} \mathrm{O}$ con resultados favorables de disociación. Acercamientos hacia enlaces paralelos al eje de la molécula del óxido. 


\section{Congresos}

I. "Interacción de $\mathrm{N}_{2} \mathrm{O}-\mathrm{Pt}_{8}$. Estudio Teórico con la aproximación cuántica relativista ZORA". V. Bertin, E. Hernández, O. Olvera-Neria, J. C. Gonzalez, E. Poulain. XXIII CICAT - Congreso Iberoamericano de Catálisis. Santa Fe Argentina, del 2 al 7 septiembre del 2012.

II. "Control catalítico de óxido nitroso". E. Hernández, E. Agacino, V. Bertin. XII Reunión de Fisicoquímica Teórica. Querétaro, del 13 al 16 de noviembre del 2013.

III. "Estudio teórico de la reacción $\mathrm{N}_{2} \mathrm{O} \rightarrow \mathrm{N}_{2}+\mathrm{O}$ catalizada por nanopartículas de Pts dopadas con O". E. Hernández, V. Bertin, E. Agacino. QUITEL-2014. Congreso de Químicos Teóricos de Expresión Latina. Isla de San Cristóbal Ecuador del 23 al 30 de noviembre del 2014. 
Esta tesis presenta un estudio teórico de las condiciones en las que se favorece la disociación de la molécula del óxido nitroso. Se usaron nanopartículas de platino de ocho átomos, en estado basal y también en los primeros estados excitados. El estudio se realizó con la Teoría de Funcionales de la Densidad, implementada en el programa ADF.

En una primera etapa del desarrollo del trabajo se optimizaron estructuras de la nanopartícula de $\mathrm{Pt}$, con el fin de encontrar una estructura más estable. También se optimizó la molécula de óxido nitroso, para determinar sus parámetros. La determinación de estos parámetros se realizó usando el programa antes mencionado, con funciones de base triple zeta y de doble polarización, con el funcional para el intercambio y correlación PBE y la aproximación de gradiente generalizado GGA. Además se incluyen los efectos relativistas con la aproximación ZORA. La estructura más estable determinada no tiene degeneración con las otras geometrías ni con otras multiplicidades de espín.

En la segunda etapa se realizó el estudio de las interacciones del óxido nitroso, sobre distintos sitios de la nanopartícula. Probando además distintas multiplicidades y diferentes modos de acercamientos. En los resultados obtenidos en las aproximaciones del óxido a la partícula $\mathrm{Pt}_{8}$ se obtuvieron pocos sitios. Las interacciones más favorables para la reacción fueron acercamientos a enlaces. La multiplicidad también fue importante, la activación se realizó para distintos valores de ésta.

En una tercera y última etapa de este trabajo, debido a los poco sitios reactivos determinados en la segunda parte, se dopó con oxígeno a la nanopartícula de platino con el fin de mejorar su rendimiento. Se realizó nuevamente la optimización de su geometría y posteriormente, se hizo el estudio de las interacciones, para caracterizar los sitios reactivos. Los resultados obtenidos fueron mejores, aumentó el número de sitios 
activos, para diferentes multiplicidades. Las interacciones más activas se lograron acercando $\mathrm{N}_{2} \mathrm{O}$ por el átomo de $\mathrm{O}$ hacia los átomos de la nanopartícula de $\mathrm{Pt}_{8} \mathrm{O}$. 


\section{Abstract}

This thesis presents a theoretical study on the conditions favoring the dissociation of the nitrous oxide molecule. The platinum nanoparticles consisting of 8 atoms were used in basal state and also in the first excited states. The study was performed using Density Functional Theory implemented in ADF program.

In the first stage of the research, Pt nanoparticle structures were optimized to find a more stable structure. The nitrous oxide molecule was also optimized to determine its parameters, and their determination was carried out using the ADF program mentioned above, with triple-zeta double polarization functions, the PBE exchange-correlation functional and generalized gradient approximation, GGA. In addition, relativistic effects with ZORA approximation were included. The most stable structure established has no degeneration with other geometries or other spin multiplicities.

In the second stage, nitrous oxide interactions were studied on different nanoparticle sites. Furthermore, different multiplicities and approximation modes were tested as well. The results obtained in the approximations of the moleculePt ${ }_{8}$ were few reactive sites. The interactions that favored the reaction were approximations to the bonds. Since the multiplicity was also important, activation was performed for different multiplicity values.

In the third and last stage of this research, due to very few reactive sites established in the second stage, the Pt nanoparticle was doped with oxygen to improve its yield. The optimization of its geometry was again performed and subsequently, the study on interactions was carried out to characterize the reactive sites. Better results were thus obtained, and the number of active sites for different multiplicities increased. The most active interactions were achieved with direct approximations of the $\mathrm{N}_{2} \mathrm{O}$ by the $\mathrm{O}$ atom towards the $\mathrm{Pt}_{8} \mathrm{O}$ nanoparticle atoms. 


\section{Hipótesis}

I. Las nanopartículas de platino aisladas catalizan la reducción del $\mathrm{N}_{2} \mathrm{O}$, el tamaño de esta influye en su reactividad, así como la naturaleza del sitio reactivo.

II. La eficiencia puede mejorarse con aditivos como átomos de oxígeno, soportes y/o cargas eléctricas.

III. La disociación de $\mathrm{N}_{2} \mathrm{O}$ depende de la forma de las partículas del catalizador, el tipo de acercamiento de las moléculas y de la multiplicidad del espín del sistema.

\section{Objetivos}

\subsection{Objetivo General}

Estudiar teóricamente la reducción del $\mathrm{N}_{2} \mathrm{O}$ catalizada por cúmulos de $\mathrm{Pt}_{8}$ y $\mathrm{Pt}_{8} \mathrm{O}$ por medio de la teoría de las funcionales de la densidad (DFT) contenido en el paquete $\mathrm{ADF}$, incluyendo efectos relativistas, necesarios para describir metales de transición.

\subsection{Objetivos Específicos}

I. Optimizar teóricamente la geometría de los cúmulos de $\mathrm{Pt}_{8}$ por medio del teoría de la DFT, para obtener los isómeros de menor energía de cada multiplicidad de espín, empleando un core congelado y 32 electrones de valencia para el átomo de Pt. 
II. Caracterizar las estructuras electrónicas de los cúmulos de menor energía, por distancia de enlace, frecuencias de vibración y multiplicidad de espín.

III. Caracterizar los tipos de interacción en que se produce la disociación del $\mathrm{N}_{2} \mathrm{O}$ con las nanopartículas de $\mathrm{Pt}_{8}$ en términos de la naturaleza de los enlaces, la forma, la orientación inicial de la molécula respecto al cúmulo de Pt, la multiplicidad de espín, las transferencias de carga eléctrica entre átomos y las frecuencias de máxima intensidad de adsorción.

IV. Evaluar el cambio en la capacidad de reducción de $\mathrm{N}_{2} \mathrm{O}$ por adición de cargas y por la adición de átomos de $\mathrm{O}$ al cúmulo. 


\section{Metodología}

Los cálculos se realizaron empleando el método ZORA implementado en el paquete Amsterdam Density Functional, ADF2013.01 [1]. Este paquete permite estudiar interacciones de metales de transición en diversas reacciones químicas, en algunos casos con mejores resultados que los métodos semiempíricos y los derivados de la teoría Hartree-Fock. El método emplea los postulados básicos de Kohn-Sham para calcular las integrales, de la DFT, pero ZORA es una aproximación de orden cero de la ecuación de Dirac, no de la de Schrödinger, por lo cual el espín es una condición para que exista la solución, en otros términos el spin sale explícitamente del método, no se impone como en la ecuación de Schrödinger.

Las aproximaciones DFT requieren de un funcional para determinar la energía de intercambio-correlación de los electrones, que la DFT postula que existe pero no se indica la manera de obtenerlo, por lo cual debe proponerse. Existe una enorme variedad de propuestas para este funcional, gran parte de las cuales incluyen parámetros empíricos apropiados al sistema que se estudia. En este estudio las energías de intercambio y correlación se calcularon con el funcional de Perdew-Burke- Ernzerhof, PBE [2], que ha sido utilizado previamente por diversos autores con la aproximación del Gradiente Generalizado, GGA. Existen otros funcionales incluso mejores, según algunos autores, que el PBE, como el PBE0, pero el cual se calcula con base de Gaussianas haciendo muy largo el cálculo con ADF que utiliza la base de Slater. En un cálculo de prueba no se encontró gran diferencia entre los resultados para el metal estudiado aquí obtenido con ambos funcionales, el sistema aplicado fue $\mathrm{Pt}_{2}$ y $\mathrm{ADF}$ con PBE0 tardó 10 veces el tiempo empleado al usar PBE, por lo cual el estudio empleó el funcional indicado.

Los métodos que emplean la DFT completa o en parte, son métodos que permiten trabajar con sistemas relativamente grandes, con un costo computacional relativamente bajo, similar al de los cálculos de HF, pero incluyendo correlación electrónica. En general, por emplear un solo determinante de Slater, los métodos DFT son más 
apropiados para caracterizar al estado basal de los sistemas, y a lo sumo puede incluir los sistemas excitados más bajos, cercanos al fundamental.

El método DFT, aplicado al estudio de sistemas en estado fundamental, proporciona buenas geometrías, momentos dipolares, frecuencias vibracionales y una buena estimación de la termodinámica y barreras de reacción con un costo computacional similar al de HF.

Entre las bases atómicas la más apropiada para este estudio es la formada por orbitales tipo Slater, incluida en el paquete $\mathrm{ADF}$, porque es una base que reproduce bien la antisimetría de la función de onda de los electrones. La base usada tiene tres funciones de base por cada orbital y 2 funciones de polarización, se simboliza TZ2P. Los átomos de $\mathrm{N}$ y $\mathrm{O}$ se describen con todos sus electrones. Para el átomo de $\mathrm{Pt}$ en cambio se emplea la aproximación de Born-Oppenheimer ó aproximación de core congelado dejando 32 electrones de valencia: $5 s^{2} 5 p^{6} 5 d^{9} 6 s^{1} 4 f^{14}$. Esta aproximación de core congelado se usa para disminuir el tiempo de computo sin perder la calidad en la representación de reacciones químicas.

Como Pt es un metal de transición del $6^{\circ}$ período con numero atómico 78 ([Xe] $\left.4 \mathrm{f}^{14} 5 \mathrm{~d}^{9} 6 \mathrm{~s}^{1}\right)$ debe incluirse en su descripción efectos relativistas, por el efecto de atracción del core hacia los e-, estos adquieren velocidades cercanas a la de la luz, por lo que es importante incluir estos efectos relativistas presentes en metales pesados como el platino, para su descripción adecuada. Estos efectos se incorporaron con ZORA que es la aproximación de orden cero a la ecuación de Dirac, adecuada por que se incorporan los parámetros escalares masa-velocidad y acoplamiento espín-orbital de Darwin $[1,4,6]$.

$$
H_{0}=H_{n r e l}=\frac{p^{2}}{2 m}+V \quad \text { Ec. } 1
$$

Donde el Hamiltoniano de orden cero es igual al Hamiltoniano de Schrödinger no relativista, donde $\boldsymbol{p}$ es el operador de momento, $\boldsymbol{m}$ la masa, $\boldsymbol{c}$ la velocidad de la luz y $\boldsymbol{V}$ 
es el potencial de Coulomb $(V=-Z / r)$. En el Anexo se describe el método con más detalle.

Para la optimización de los diferentes cúmulos de platino se proponen diferentes geometrías, las cuales se optimizan sin restricción de simetría para encontrar la estructura más estable. Realizada la optimización geométrica se hace el cálculo de los modos vibracionales para confirmar que efectivamente corresponde al mínimo global en la superficie de energía potencial. La geometría de las partículas $\mathrm{Pt}_{8}$ y $\mathrm{Pt}_{8} \mathrm{O}$, se optimizaron sin ningún tipo de restricción para cuatro multiplicidades de espín, 1, 3, 5, 7, para determinar el estado basal y los estados excitados probables.

La energía de enlace de los cúmulos se determinó con la ecuación:

$$
B E={ }^{M} E_{P t_{8}}-8^{1} E_{P t}
$$

Donde $B E$ es la energía de enlace, ${ }^{M} E_{P t 8}$ es la energía obtenida con el método ZORA-DFT del paquete de cálculo ADF para el cúmulo aislado y ${ }^{1} E_{P t}$ es la energía obtenida para un átomo aislado de Pt en su estado basal.

La energía de disociación $E_{d i s}$ de la nanopartícula de $\mathrm{Pt}_{8}$ del estado fundamental con multiplicidad de espín triplete y con $\mathrm{N}_{2} \mathrm{O}$ con multiplicidad de espín singulete se calculó con la ecuación:

$$
E_{d i s}={ }^{M} E_{P_{8} N_{2} O}-\left({ }^{3} E_{P_{8}}+{ }^{1} E_{N_{2} O}\right)
$$

Donde ${ }^{M} E_{P t 8-N 2 O}$ es la energía del cúmulo con la molécula de óxido nitroso calculado para diferentes multiplicidades de espín (M), ${ }^{3} E_{P t 8}$ la energía de la nanopartícula de platino de ocho átomos y ${ }^{1} E_{N 2 O}$ la energía de la molécula del óxido nitroso, estos últimos optimizados previamente.

La oxidación del cúmulo de platino se realiza sin restricción de simetría. La energía de oxidación para este nuevo isómero oxidado se determina con la siguiente relación: 


$$
E_{\text {oxidación }}={ }^{M} E_{P_{8} O}-\left({ }^{3} E_{P_{8}}+\frac{1}{2}{ }^{1} E_{O_{2}}\right)
$$

Donde ${ }^{3} E_{P t 8}$ es la energía del cumulo sin oxidar, ${ }^{1} E_{O 2} / 2$ la energía del átomo de oxigeno y ${ }^{M} E_{P t 8 O}$ la energía de cumulo oxidado calculado para diferentes multiplicidades de espín (M).

Para determinar la energía de disociación $E_{d i s}$ de la nanopartícula oxidada con $\mathrm{N}_{2} \mathrm{O}$ se determinó con la siguiente ecuación:

$$
E_{d i s}={ }^{M} E_{P_{8} O-N_{2} O}-\left({ }^{1} E_{P_{8} O}+{ }^{1} E_{N_{2} O}\right)
$$

Donde ${ }^{M} E_{P t 8 O-N 2 O}$ es la energía del cúmulo oxidado con la molécula del óxido nitroso calculado para diferentes multiplicidades de espín (M), ${ }^{I} E_{P t 8 O}$ es la energía de la nanopartícula oxidada de platino con ocho átomos y ${ }^{1} E_{N 2 O}$ es la energía de la molécula del óxido nitroso, estos últimos optimizados previamente.

Para determinar la distribución electrónica en todos los átomos se realizó un análisis de población, que da la distribución de cargas dentro del átomo. El método empleando fue esquema propuesto por Hirshfeld cuya ecuación se muestra a continuación.

$$
\begin{gathered}
Q_{j}=Z_{j}-\int_{v} \rho(\vec{r}) w_{A} \\
=Z_{j}-\int_{v} \rho(\vec{r}) \frac{\rho_{A}(\vec{r})}{\sum_{j} \rho_{A}(\vec{r})}
\end{gathered}
$$

Ec. 6

Este se basa en el uso de densidades atómicas $\rho_{A}(\vec{r})$ para la partición de la densidad electrónica molecular $\rho(\vec{r})$ del sistema total en equilibrio. La densidad de electrones 
real en cada punto en el espacio se reparte entre los átomos por factores de ponderación $w_{A}$ de acuerdo con las contribuciones atómicas, $Z_{j}$ es la carga del núcleo j. 


\section{Antecedentes}

\subsection{El contaminante $\mathrm{N}_{2} \mathrm{O}$}

El $\mathrm{N}_{2} \mathrm{O}$, con otros óxidos de nitrógeno son productos naturales, producidos por la descomposición bacteriana de nitratos orgánicos, procesos biogeoquímicos, como las erupciones volcánicas y otros que ocurren en el ciclo del nitrógeno.

En la actualidad la fuente principal en la emisión a la atmósfera de óxidos de nitrógeno es el excesivo uso de fertilizantes nitrogenados sintéticos en la agricultura, el aumento del estiércol del ganado y la creación de nuevas tierras agrícolas a partir de bosques y praderas, liberando nitrógeno en el suelo. En menor porcentaje, contribuyen a liberar óxidos de nitrógeno las industrias productoras de nylon, amoníaco y ácido nítrico, así como centrales eléctricas y vehículos que emplean combustibles derivados del petróleo [7-9].

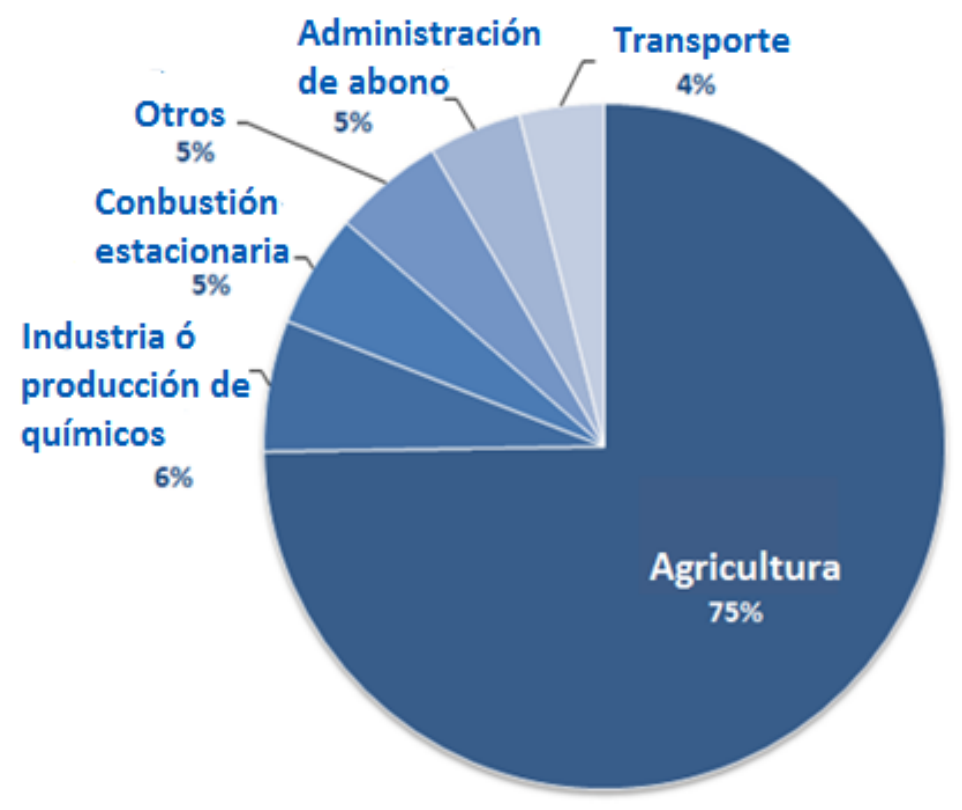

Figura 4.1.1 Emisiones de $\mathrm{N}_{2} \mathrm{O}$ enU.S. Fuente: Inventory of U.S. Greenhouse Gas Emissions and Sinks: 1990-2012. 
Los óxidos de nitrógeno $\left(\mathrm{NO}_{\mathrm{x}}\right)$, junto con $\mathrm{CO}_{2}$, óxidos de azufre y compuestos orgánicos volátiles son los contaminantes primarios del aire. Los principales óxidos de nitrógeno presentes en la atmosfera son: óxido nítrico ( $\mathrm{NO}$ ), dióxido de nitrógeno $\left(\mathrm{NO}_{2}\right)$ y óxido nitroso $\left(\mathrm{N}_{2} \mathrm{O}\right)$. El óxido nitroso se produce en cantidades mucho menores que los otros dos, pero se produce en las reducciones incompletas de los otros óxidos, es por lo tanto la etapa anterior en la reducción a $\mathrm{N}_{2}$ de los $\mathrm{NO}_{\mathrm{x}}$. $\mathrm{El} \mathrm{N}_{2} \mathrm{O}$ es un potente gas de efecto invernadero debido a que estas moléculas tienen mayor capacidad que el $\mathrm{CO}_{2}$ para absorber radiación infrarroja, aumentando considerablemente la temperatura de la tropósfera [8].

La temperatura en promedio de la Tierra aumentó unos $0.6^{\circ} \mathrm{C}\left( \pm 0.2^{\circ} \mathrm{C}\right)[7]$ durante el siglo pasado, por lo cual las capas terrestres de hielo han disminuido, el nivel de los océanos está aumentando y el oxígeno disuelto en el agua de lagos y océanos está disminuyendo rompiendo cadenas alimenticias. Hasta hace pocos años se creía que el calentamiento global era provocado por el crecimiento en la atmósfera del $\mathrm{CO}_{2}$ y el $\mathrm{CH}_{4}$ provocado por la actividad humana, pero hoy se sabe que la potencia del $\mathrm{N}_{2} \mathrm{O}$ para producir el efecto invernadero es 260 veces mayor que la del $\mathrm{CO}_{2}$ [7]. La cantidad del $\mathrm{N}_{2} \mathrm{O}$ en la tropósfera se mantuvo constante por miles de años, unas $280 \mathrm{ppb}$, con el desarrollo industrial empezó su aumento que hoy es entre 0.2 y $0.3 \% / a n ̃ o$, ya son millones de toneladas las emitidas a la troposfera[9].

El $\mathrm{N}_{2} \mathrm{O}$ juega un papel importante en las reacciones químicas que generan smog fotoquímico y ozono a nivel de suelo y que en la estratósfera destruyen al ozono [4], así como también contribuye al efecto de la lluvia acida. El tiempo de residencia en la atmósfera del óxido nitroso se ha estimado en 120 años [9], descomponiéndose finalmente en la estratósfera por reacciones fotolíticas. Además el dióxido de nitrógeno es un fuerte agente oxidante, que reacciona en el aire para formar ácido nítrico corrosivo, así como nitratos orgánicos tóxicos. En concentraciones significativas, el $\mathrm{N}_{2} \mathrm{O}$ es altamente tóxico. La regulación en las emisiones de este gas se ha introducido progresivamente en la mayor parte delospaíses de todo el mundo. Nuevas tecnologías se han introducido para bien limitar su formación o convertirlos a $\mathrm{N}_{2}$ [7]. 
Por los peligros del calentamiento global los países han venido tomando acuerdos para disminuir los efectos de los contaminantes sobre el clima y sobre la capa de ozono, como los acuerdos de los Protocolos de Kyoto y Montreal [11,12], y reuniones posteriores para ir implementando los acuerdos de estos eventos. El de Kyoto se adoptó en 1997 pero sólo se empezó a aplicar en 2005, su objetivo es reducir las emisiones de 6 gases de efecto invernadero, $\mathrm{CO}_{2}, \mathrm{CH}_{4}, \mathrm{~N}_{2} \mathrm{O}, \mathrm{HFC}, \mathrm{PFC}$ y SF 6 . El de Montreal es un tratado internacional [11] para proteger la capa de ozono de la estratósfera, se aplica desde 1989 y es un ejemplo de cooperación internacional para terminar con un problema que puede afectar la supervivencia. En México se realizó la XV Conferencia sobre el Cambio Climático en Cancún, 2010, en que participaron más de 190 países. Países como Estados Unidos, el mayor emisor de gases de efecto invernadero no lo ha ratificado. Canadá lo firmó pero no lo ratificó. Gran parte del resto del mundo lo firmó y ratificó, pero las implementaciones se han ido realizando en forma más lenta de lo acordado, a diferencia de lo ocurrido con los acuerdos de Montreal.

\subsection{Descripción de las reacciones en dispositivos catalíticos.}

La reducción de los óxidos de nitrógeno $\left(\mathrm{NO}_{\mathrm{X}}\right)$ a $\mathrm{N}_{2}$ y la oxidación de $\mathrm{CO}$ a $\mathrm{CO}_{2}$ son procesos deseables, porque reducen la contaminación. Para estos procesos se utilizan catalizadores en los que participan metales de transición como Rh, Pd y Pt $[7,13]$, que deben estar muy dispersos, es decir deben estar formados por partículas metálicas pequeñas. Los contaminantes se adsorben sobre las partículas e interaccionan entre sí. La reducción de óxidos de nitrógeno a $\mathrm{N}_{2}(\mathrm{~g})$ no es un proceso sencillo. En la literatura se encuentran muchas propuestas [13-24]. Wiessen [7, Capítulo 9] indica que los óxidos de nitrógeno, en los procesos de combustión en vehículos con motor de

combustión de hidrocarburos pasan más fácilmente a $\mathrm{N}_{2} \mathrm{O}$ que a $\mathrm{N}_{2}$, durante la etapa del calentamiento. Por lo tanto $\mathrm{N}_{2} \mathrm{O}$ es un intermediario en la reducción del NO. En los 
procesos de descontaminación la formación de $\mathrm{N}_{2} \mathrm{O}$ no es lo adecuado porque este óxido es también un contaminante, por lo cual es necesario determinar las condiciones para que la reacción de reducción de $\mathrm{NO}$ se complete y termine en la formación de $\mathrm{N}_{2}$.

Winter a finales de 1960 [22-24] propone el siguiente mecanismo general para la descomposición de $\mathrm{N}_{2} \mathrm{O}$.

$$
\begin{aligned}
& \mathrm{N}_{2} \mathrm{O}+* \rightarrow \mathrm{N}_{2}+\mathrm{O}_{(a d s)} \\
& 2 \mathrm{O}_{(a d s)} \leftrightarrow \mathrm{O}_{2}+2 * \\
& \mathrm{~N}_{2} \mathrm{O}+\mathrm{O}_{(a d s)} \rightarrow \mathrm{N}_{2}+\mathrm{O}_{2}
\end{aligned}
$$

Rh es el metal que se señala como esencial para la reducción de los óxidos de nitrógeno en los catalizadores de tres vías de los vehículos [25,26], porque se reducen sobre el metal incluso en ausencia de otro reductor, como $\mathrm{CO}$ o $\mathrm{H}_{2}$. Es un metal bastante más caro (Figura 1.2.1) que la mayoría de los metales de transición, por lo cual es interesante buscar condiciones para obtener la reducción de uno o varios óxidos de nitrógeno, con buen rendimiento, sobre otro metal.

Como $\mathrm{N}_{2} \mathrm{O}$ ha sido señalado como intermediario $\mathrm{y} / \mathrm{o}$ subproducto en la reducción de $\mathrm{NO}$ a $\mathrm{N}_{2}$, en este trabajo se buscan condiciones para obtener la reducción de este óxido sobre partículas de Pt. Estudios experimentales [21] han demostrado al Pt efectivo en la reducción de estos óxidos comparada con otros grupos de metales [22], por lo que se usará como alternativa en sustitución del Rh para ser utilizado en convertidores catalíticos de automóviles, para reducir los contaminantes tóxicos, tales como el $\mathrm{CO}, \mathrm{NO}_{\mathrm{x}}$ e hidrocarburos .

*Autor correspondiente: R.Burch (E-mail:R.Burch@qub.ac.uk) 


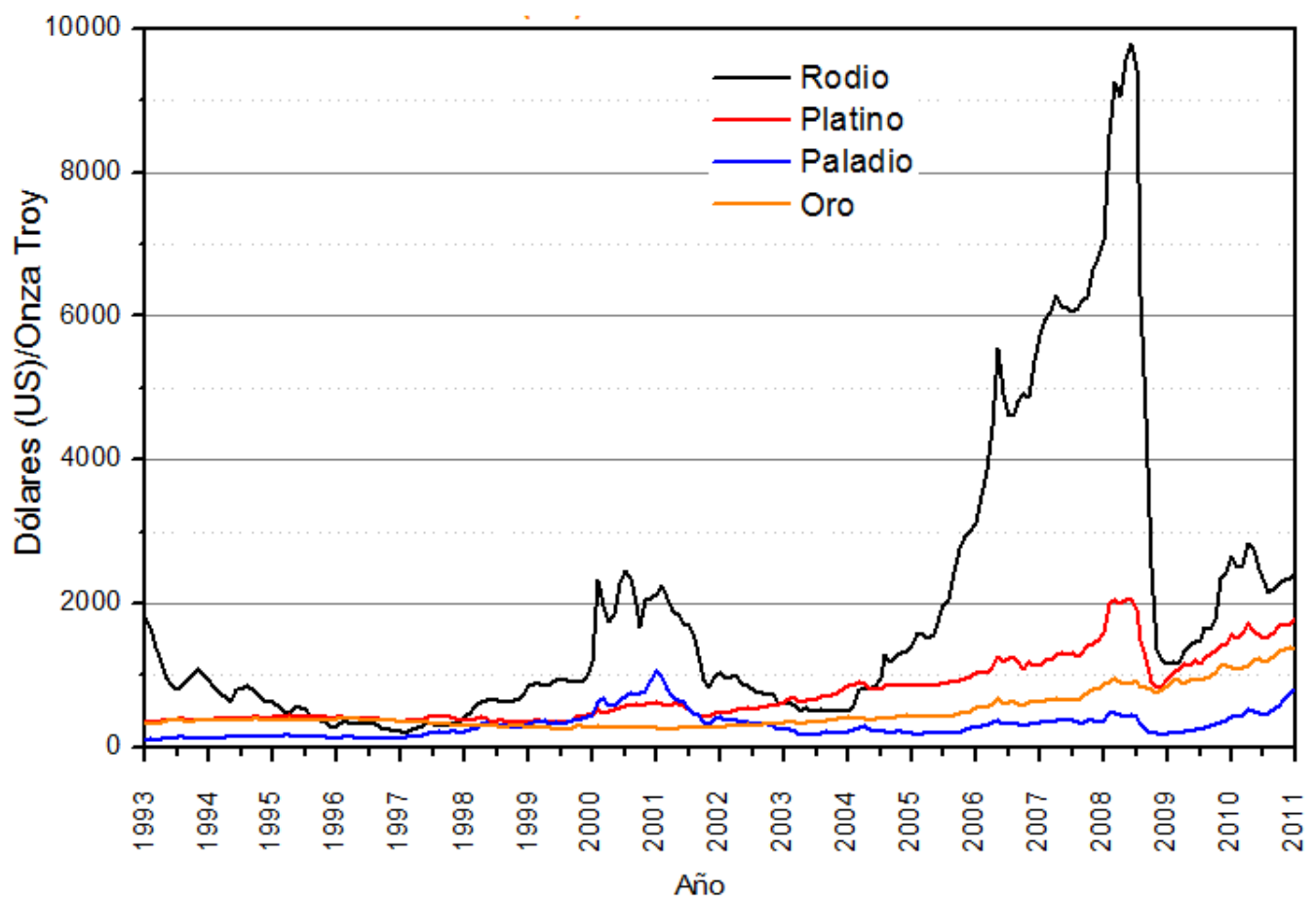

Figura 4.2.1 Evolución del precio de los metales de transición usados en convertidores catalíticos: Rodio, Platino, Paladio y Oro. versus Precio en dólares (US)/onzaTroy. (1onza troy=31.1035gr ) Fuente: www.platinum.matthey.com $(\mathrm{Ph}, \mathrm{Pt}, \mathrm{Pd})$; www.kitco.com (Au)

La concentración de $\mathrm{N}_{2} \mathrm{O}$ es típicamente baja a temperaturas inferiores a la activación del Rh, en el gas emitido por motores de combustión de hidrocarburos, pero en la etapa de calentamiento, entre 60 y $80 \%$ de $\mathrm{NO}$ se reduce parcialmente a $\mathrm{N}_{2} \mathrm{O}$ que se libera a la atmósfera. Para aumentar el rendimiento de la reducción es necesario aumentar los conocimientos sobre la reducción de $\mathrm{N}_{2} \mathrm{O}$ en condiciones suaves de funcionamiento, en la etapa de precalentamiento del motor, sobre otros metales, como $\mathrm{Pt}$, sobre el cual se produce justo sobre $300^{\circ} \mathrm{C}$, así como a una temperatura aún alta y con menor rendimiento que $\mathrm{Rh}$ [27,28]. En la referencia [29] Hermes y col. sugieren que la adición de $\mathrm{O}$ a la nanopartícula de $\mathrm{Rh}_{5}{ }^{+}$promueve la descomposición de $\mathrm{N}_{2} \mathrm{O}$.

Kapteijn et al. [13] realizaron en 1966 una amplia y muy buena revisión de los sistemas catalíticos empleados para descomponer $\mathrm{N}_{2} \mathrm{O}$. Señalan que se ha empleado muy diversos catalizadores, por ejemplo de Pt y Au como metales puros, además de 60 óxidos puros, como $\mathrm{MgO}, \mathrm{CuO}, \mathrm{Lu}_{2} \mathrm{O}_{3}, \mathrm{CaO}, \mathrm{Al}_{2} \mathrm{O}_{3}, \mathrm{y}$ también mezclas de óxidos, 
perovskitas, zeolitas y sistemas soportados. Los catalizadores se han empleado en diferentes intervalos de temperatura, entre 400 y más de $1000 \mathrm{~K}$. Algunos catalizadores de Pt trabajan en presencia de $\mathrm{O}_{2}$. Entre sus conclusiones expresan que las actividades catalíticas deben mejorarse. La descomposición de $\mathrm{N}_{2} \mathrm{O}$ es factible termodinámicamente $(\Delta \mathrm{G}<0)$.

Es necesario bajar las temperaturas de activación del catalizador, para evitar que los sistemas emitan al óxido mientras se calienta el sistema. Además deben complementarse con otros sistemas para evitar el envenenamiento del catalizador, por ejemplo con $\mathrm{SO}_{2}$. A la fecha el número de sistemas catalíticos para esta reducción ha aumentado mucho, ver por ejemplo la Tabla 1 de la referencia [9] que incluye más metales puros, como $\mathrm{K}, \mathrm{Mg}, \mathrm{Rh}$, $\mathrm{Ru}$, etc. Además las concentraciones de $\mathrm{N}_{2} \mathrm{O}, \mathrm{N}_{2}$ y $\mathrm{O}_{2}$ oscilan isotérmicamente durante la reducción del óxido sobre ciertos catalizadores como $\mathrm{Cu} / \mathrm{ZSM}-5$.

Actualmente se están desarrollando procesos de descomposición fotoquímica para superar problemas presentados por la catálisis normal.

\subsection{Estudios teóricos sobre nanopartículas de Pt}

Las nanopartículas de platino han sido estudiadas teóricamente por diversos autores [38-44, 48-55], pero aún no se han logrado obtener resultados concordantes [44] respecto a la energía y estructura geométrica del estado fundamenta de los cúmulos de diferente tamaño.

Xiao y Wang [40] encuentran energías parecidas e incluso menores hasta $\mathrm{Pt}_{6}$ en estructuras planas que tridimensionales, empleando DFT con un conjunto de base de onda plana implementado en el paquete de simulación Viena Ab initio (VASP). Para $\mathrm{Pt}_{8}$ las 3 estructuras optimizadas son de tipo cúbico, con deformaciones y sus energías son muy parecidas. Los cúmulos de $\mathrm{Pt}_{2} \mathrm{a} \mathrm{Pt}_{6}$ fueron estudiados por Bloński et al. [48] encuentran que hasta $\mathrm{Pt}_{5}$ son más estables las estructuras planas y para $\mathrm{Pt}_{6}$ son más 
estables las tridimensionales, sus cálculos están basados en la DFT implementado en el VASP y un conjunto de base de onda plana. Huda et al. [43] estudian estructuras de $\mathrm{Pt}_{2}$ a $\mathrm{Pt}_{5}$ usando el método PAW (Projected Augmented Wave) junto con VASP (Viena ab initio simulation package) determinando que para $\mathrm{Pt}_{5}$ la estructura plana cuadrada con un átomo sobre uno de los enlaces es más estable que la bipirámide triangular y la pirámide cuadrada, con ó sin acoplamiento espín-órbita, aunque estas dos últimas son más estables que el resto de estructuras planas propuestas. Otros autores en cambio, con el paquete de cálculo DMol [42], usan la aproximación del gradiente generalizado con el funcional Perdew-Wang de intercambio y correlación, realizan optimizaciones sin restricciones. No mencionan correcciones relativistas, como también es el caso de las referencias anteriores. Estos autores encuentran que $\mathrm{Pt}_{4} \mathrm{y} \mathrm{Pt}_{5}$ tridimensionales son más estables que las estructuras planas, $\mathrm{Pt}_{6}$ es plano pero de $\mathrm{Pt}_{7}$ hasta $\mathrm{Pt}_{38}$, todas las estructuras son tridimensionales. En la Figura 9 comparan estructuras de $\mathrm{Pt}_{8} \mathrm{y} \mathrm{Pt}_{9}$. Para $\mathrm{Pt}_{8}$ la estructura más estable es una bipirámide pentagonal con un átomo sobre una de las caras, que es $-3.5 \mathrm{kcal} / \mathrm{mol}$ más estable que la cúbica que otros autores ya mencionados han propuesto como la más estable. Bhattacharyya y Majumder [49] proponen una estructura plana, con 2 átomos sobre enlaces de una estructura triangular, como más estable que las cúbicas regular y asimétrica, correspondiendo por lo tanto al estado fundamental (Energía de enlace $=\mathrm{BE}=621.7 \mathrm{kcal} / \mathrm{mol}$ ). Al aumentar la contribución del orbital $\mathrm{p}$ se produciría la transición de estructuras planas a tridimensionales (Cálculos realizados con el paquete GGA-VASP).

Kumar y Kawazoe [50] proponen estructuras de cúmulos de $\mathrm{Pt}_{\mathrm{n}}$ desde $\mathrm{n}=2$ a 38, algunas planas. Para $\mathrm{Pt}_{8}$ obtienen 4 geometrías, dos son planas, una de estas se obtuvo al optimizar una estructura hexagonal centrada y coronada. Para estos autores el estado basal es una bipirámide hexagonal de multiplicidad 3. La geometría más cercana es plana, son dos cuadrados bicoronados también de $\mathrm{M}=3$ y a $3.23 \mathrm{kcal} / \mathrm{mol}$ sobre la bipirámide. Las otras geometrías, ambas quintupletes, están a 4.01 y 7.38 por encima de la basal. El método empleado es el pseudo potencial ultra suave (para estructuras iníciales) y el de onda proyectada aumentada para las geometrías seleccionadas, (USP y PAW por sus nombres en inglés), con el gradiente generalizado de espín polarizado. Las optimizaciones no tienen restricciones. 
Wang et al. [51] estudian cúmulos de Pt de 15 a 24 átomos empleando DFT y un algoritmo generico (GA). Calculan también $\mathrm{Pt}_{2}$ para comparar con resultados experimentales. Sus resultados son muy cercanos a los experimentales, $\mathrm{d}_{\mathrm{Pt}-\mathrm{Pt}}$ es de 2.39 $\AA$ versus $2.34 \AA$ [31], EB $-3.59 \mathrm{eV}$ contra -3.14 [32], frecuencia vibracional $215.6 \mathrm{~cm}^{-1}$ calculada $217.2 \mathrm{~cm}^{-1}$ y experimental [32].

Heredia et al. [52] obtienen para $\mathrm{Pt}_{8}$ una $\mathrm{BE}$ de -2.58 eV/átomo (487.29 $\mathrm{kcal} / \mathrm{mol}$ ) y đ đet-Pt de $2.702 \AA$. La geometría es un octaedro con dos Pt coronando dos caras, arriba y abajo del ecuador. El método empleado es el paquete Gaussian03, el funcional es B3PW91. Posteriormente estos autores [53] con la misma metodología determinan que la estructura de $\mathrm{Pt}_{8}$ es cúbica, con $\mathrm{BE}$ de $-2.72 \mathrm{eV} /$ átomo (-513.73 $\mathrm{kcal} / \mathrm{mol})$ y $\mathrm{M} 9$.

La estructura de $\mathrm{Pt}_{8}$ ha sido también estudiada por Sebetci [54-56]. En un primer trabajo realizado con Güvenç en 2003, empleando dinámica molecular y la simulación de enfriamiento térmico, la estructura propuesta, entre otras, es un octaedro bicoronado, con los 2 átomos sobre diversas caras del octaedro, una de las cuales es la geometría del basal con energía de enlace $(\mathrm{BE})$ de $-736.10 \mathrm{kcal} / \mathrm{mol}$, y đ đt-Pt promedio de $2.52 \AA$. Posteriormente en 2013 este autor, empleando el método DFT de espín polarizado del paquete NWChem-6.0, con la aproximación GGA del funcional BPW91, propone 12 estructuras geométricas para $\mathrm{Pt}_{8}$, entre las cuales el basal corresponde a un triplete con geometría que llama pirámide triple tetragonal, degenerado con un quintuplete de un prisma trigonal bicoronado en dos caras, una rectangular y la otra triangular. Estas dos geometrías tienen $\mathrm{BE}$ de $-577.44 \mathrm{y}-577.07 \mathrm{kcal} / \mathrm{mol}(\Delta \mathrm{BE}=0.37 \mathrm{kcal} / \mathrm{mol})$. Las frecuencias vibracionales de estos estados son 213 y $241 \mathrm{~cm}^{-1}$ respectivamente y los “gap” HOMO-LUMO son 5.30 y $4.38 \mathrm{kcal} / \mathrm{mol}$.

Existen pocos estudios experimentales sobre partículas de $\mathrm{Pt}$, los cuales corresponden a estudios espectroscópicos que permiten determinar estados basales y frecuencias vibracionales de cúmulos muy pequeños, como $\mathrm{Pt}_{2}$ y $\mathrm{Pt}_{3}$, neutros o con cargas negativas [31-37]. 
Muchos de los resultados que se encontraron emplean la teoría de funcionales de la densidad (DFT) como los descritos anteriormente, pero los resultandos no concuerdan por varias razones, una de ellas es el empleo de diferentes funcionales para el cálculo de la energía de intercambio-correlación ( $\mathrm{E}_{\mathrm{XC}}$ ), además de no incluir los efectos relativistas que presentan los metales pesados como el Pt.

En este trabajo se emplea el funcional PEB que es adecuado para describir las propiedades de los metales y así como la aproximación ZORA para los efectos relativistas, como se describió anteriormente en la metodología. 


\section{Resultados y discusión}

\subsection{Propiedades químicas de platino $\mathrm{Pt}_{2}$ y $\mathrm{N}_{2} \mathrm{O}$}

El platino es un metal muy utilizado para fabricar catalizadores que se utilizan en diversos procesos industriales pero también para bajar los índices de contaminación atmosférica, en especial en la catálisis de $\mathrm{CO}, \mathrm{NO}_{\mathrm{x}}$ e hidrocarburos [7]. Resultados experimentales sólo se reportan para nanopartículas de $\mathrm{Pt}_{2}$ y $\mathrm{Pt}_{3}$ [30-37], en su mayoría solo reportan análisis de frecuencias de vibración. No se han encontrado trabajos experimentales que determinen propiedades de $\mathrm{Pt}_{2} \mathrm{y} \mathrm{Pt}_{3}$ con la misma técnica y la misma incertidumbre. Por lo tanto no se pueden comparar apropiadamente los resultados con los calculados en este trabajo.

En la presente tabla se muestran los resultados calculados empleando la Teoría de Funcionales de la Densidad (DFT), para nanopartículas de platino de dos átomos, usando la metodología descrita anteriormente y para los valores más bajos de multiplicidad de espín.

Tabla 5.1.1 Parámetros principales de $\mathrm{Pt}_{2}$ calculados. Energías de enlace (BE), energías relativas $\left(E_{\text {relativa }}\right)$, frecuencias de vibración $(v)$ y distancias de enlace $\left(d_{P t-P t}\right)$.

\begin{tabular}{ccccc}
\hline \hline $\mathbf{M}$ & $\begin{array}{c}\mathbf{B E} \\
\mathbf{k c a l} / \mathbf{m o l}\end{array}$ & $\begin{array}{c}\mathbf{E}_{\text {relativa }} \\
\mathbf{k c a l} / \mathbf{m o l}\end{array}$ & $\begin{array}{c}\mathbf{u} \\
\mathbf{c m}^{-\mathbf{1}}\end{array}$ & $\begin{array}{c}\mathbf{d}_{\mathbf{P t}-\mathbf{P t}} \\
\mathbf{A}\end{array}$ \\
\hline \hline 1 & -76.26 & 12.53 & 233 & 2.34 \\
$\mathbf{3}$ & $\mathbf{- 8 8 . 7 9}$ & $\mathbf{0 . 0 0}$ & $\mathbf{2 3 3}$ & $\mathbf{2 . 3 4}$ \\
5 & -68.40 & 20.39 & 237 & 2.32 \\
7 & -55.15 & 143.94 & 231 & 2.35 \\
\hline \hline
\end{tabular}

El estado basal es un triplete con $\mathrm{BE}$ de $-88.79 \mathrm{kcal} / \mathrm{mol}$, no está degenerado con los estados de otras multiplicidades y la distancia de enlace es $2.34 \AA$. Los resultados teóricos de la Energía de enlace (BE) se comparan con los de otros autores y los experimentales. 
En la Tabla 5.1.2 puede apreciarse que los cuatro trabajos seleccionados también encuentran que el estado basal tiene multiplicidad 3. Los valores de BE dependen del método utilizado. El valor determinado en este trabajo para $\mathrm{Pt}_{2} \mathrm{y}$ multiplicidad 3 es uno de los alejados del valor experimental con esa misma multiplicidad de espín. El valor reportado en ref. [31] es el más cercano al valor calculado para multiplicidad 3 pero en esta referencia se encuentra que su estado menor energía es para M 1.

Tabla 5.1.2 Comparación de resultados teóricos y experimentales para Pt ${ }_{2}$. Energías de enlace en $\mathrm{kcal} / \mathrm{mol}$.

\begin{tabular}{cccccccc}
\hline \hline $\mathbf{M}$ & $\begin{array}{c}\mathbf{B E} \\
\mathbf{k c a l} / \mathbf{m o l}\end{array}$ & $\begin{array}{c}\mathbf{B E} \\
\mathbf{k c a l} / \mathbf{m o l}\end{array}$ & $\begin{array}{c}\mathbf{B E} \\
\mathbf{k c a l} / \mathbf{m o l}\end{array}$ & $\begin{array}{c}\mathbf{B E} \\
\mathbf{k c a l} / \mathbf{m o l}\end{array}$ & $\begin{array}{c}\mathbf{B E} \\
\mathbf{k c a l} / \mathbf{m o l}\end{array}$ & $\begin{array}{c}\mathbf{B E} \\
\mathbf{k c a l} / \mathbf{m o l}\end{array}$ & $\begin{array}{c}\mathbf{B E}^{\text {Exp. }} \\
\mathbf{k c a l} / \mathbf{m o l}\end{array}$ \\
\hline \hline 1 & $-76.26^{*}$ & & & & & & $-85.5^{[31]}$ \\
3 & $-88.79^{*}$ & $-46.12^{[38]}$ & $-76.10^{[39]}$ & $-81.17^{[40]}$ & $-37.68^{[42]}$ & $-78.13^{[43]}$ & $-72.41^{[33]}$ \\
5 & $-68.40^{*}$ & & & & & & --- \\
7 & $-55.15^{*}$ & & & & & -- \\
\hline \hline * Valores calculados en este trabajo. & & & &
\end{tabular}

*Valores calculados en este trabajo.

En la Ref. [33]. se presentan otros resultados experimentales $\mathrm{BE}=-72.421 \mathrm{kcal} / \mathrm{mol}$, con $\AA_{\mathrm{Pt}-\mathrm{Pt}}=2.33 \AA$. En este trabajo se asigna la multiplicidad triplete al estado basal de $\mathrm{Pt}_{2}$. Prácticamente todos los autores obtienen triplete para el estado basal y no informan sobre otros estados. Con ADF las energías resultan mayores que con otros métodos. Este resultado se ha obtenido en otros estudios realizados con ADF de partículas pequeñas por alguno error del método las energías obtenidas están por arriba de las que reportan en otros trabajos con otras metodologías [44] y por consiguiente del valor experimental como se puede ver en la tabla anterior.

Con respecto a la molécula de $\mathrm{N}_{2} \mathrm{O}$ los resultados experimentales son confiables. En la Figura 5.1.1 se muestra la geometría de la molécula de óxido nitroso obtenida con la metodología empleada en este trabajo con la multiplicidad de espín que genera la energía más baja (singulete). 


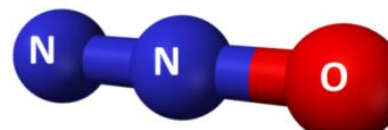

Figura 5.1.1 Geometría optimizada de la molécula de $\mathrm{N}_{2} \mathrm{O}$ (singulete).

La geometría es lineal para la multiplicidad de espín singulete por que genera la estructura más estable de la molécula de $\mathrm{N}_{2} \mathrm{O}$. Los parámetros más importantes de la optimización de esta molécula obtenidos por nuestro método de cálculo se resumen en la Tabla 5.1.3, donde también se hace una comparación con algunos valores experimentales reportados en la literatura $[30,45]$.

Tabla 5.1.3 Principales parámetros de la molécula del $\mathrm{N}_{2} \mathrm{O}$. Distancias de enlace $\left(d_{N-N}, d_{N-O}\right)$, frecuencias de vibración (v) y cargas Hirshfeld, calculados y experimentales.

\begin{tabular}{|c|c|c|c|c|}
\hline $\begin{array}{c}\text { Parámetros } \\
\text { de } \mathrm{N}_{2} \mathrm{O}\end{array}$ & $\underset{\AA}{\stackrel{\mathbf{d}_{\mathrm{N}-\mathrm{N}}}{ }}$ & $\stackrel{\mathbf{d}_{\mathrm{N}-\mathrm{O}}}{\AA}$ & $\begin{array}{c}U \\
\mathrm{~cm}^{-1}\end{array}$ & $\begin{array}{c}\text { Cargas Hirshfeld } \\
\mathrm{e}^{-} \\
\end{array}$ \\
\hline \multirow{3}{*}{ Experimentales } & \multirow{3}{*}{$1.126^{[30]}$} & \multirow{3}{*}{$1.186^{[30]}$} & $2224.2^{[45]}$ & \\
\hline & & & $1285.3^{[45]}$ & No hay valor \\
\hline & & & $589.2^{[45]}$ & \\
\hline \multirow{3}{*}{ Calculados } & \multirow{3}{*}{1.137} & \multirow{3}{*}{1.192} & 2276.0 & $\mathrm{~N}_{1}(-0.0844)$ \\
\hline & & & 1296.7 & $\mathrm{~N}_{2}(0.1963)$ \\
\hline & & & & $\mathrm{O}(-0.1119)$ \\
\hline
\end{tabular}

Se puede apreciar en la Tabla 5.1.3 que el método empleado reproduce acertadamente las propiedades de este óxido, por ejemplo, las distancias de enlace difieren de las experimentales en $0.97 \%$ para $\mathrm{N}-\mathrm{N}$ y $0.8 \%$ para $\mathrm{N}-\mathrm{O}$ (Sutton Tables [30]). Las dos frecuencias de vibración experimentales son menores a las calculadas en menos de $2.3 \%$. Su polaridad debe ser relativamente baja debido a que sus enlaces tienen momentos dipolares opuestos, de diferente magnitud; experimentalmente el 
momento dipolar es 0.161 D [46]. En su estado fundamental la molécula es lineal, como se describe experimentalmente [30].

\subsection{Reacción de disociación del $\mathrm{N}_{2} \mathrm{O}$ catalizada por nanopartículas de platino $\mathrm{Pt}_{8}$}

Para lograr superar los problemas presentados al inicio de la descomposición catalítica del $\mathrm{N}_{2} \mathrm{O}$ por los catalizadores actuales usados en motores de combustión de hidrocarburos, es necesario seguir realizando estudios para determinar los pasos relevantes de los diferentes sistemas. El Pt sigue usándose en el catalizador de tres vías así como el Rh pero por sus costos tan elevados se hace necesario buscar alternativas con otro metal como Pt, por lo cual en este estudio se caracterizan nanopartículas de este metal. I. Balteanu et. [57] por un lado y Hirotaka y col. [17] por otro lado determinan que los clústeres de 7 u 8 átomos de platino presentan mejores rendimientos en la reducción de $\mathrm{N}_{2} \mathrm{O}$ que los de tamaño pequeño. Aunque también es posible que las partículas del catalizador sean un poco mayores, las nanopartículas de 12 a 20 átomos muestran buenos rendimientos, pero por razones de tiempo de los cálculos se decidió hacer el trabajo con partículas $\mathrm{Pt}_{8}$. Tanto el tiempo como costo computacional se incrementa notablemente al incrementar el número de átomos en la nanopartícula.

Además las partículas mayores empiezan a tener propiedades metálicas lo que baja su capacidad catalítica. En Rh se ha estimado que las partículas de 60 átomos ya tienen enlace metálico [47].

En este trabajo de tesis se realizó un estudio teórico de las estructuras geométricas $\mathrm{Pt}_{8}$ y de la reacción con $\mathrm{N}_{2} \mathrm{O}$, se determina su geometría de estado fundamental y se caracterizan varios tipos de interacción con el óxido nitroso. 


\subsubsection{Optimización geométrica de las nanopartículas de Pt. Estructuras geométricas y energéticas.}

En este trabajo se partieron de estructuras tanto planas (isómero IV), como con geometría tridimensional como se muestra en la Figura 5.2.1.1 (isómeros I, II, III, V y VI). Las estructuras geometrías iníciales se tomaron en general de la literatura pero la optimización de sus geométricas al igual que los valores de energía se realizaron usando el paquete ADF y aproximación ZORA/PBE, metodología descrita previamente.

La geometría del "isómero I" propuesta en este trabajo, su geometría inicial consiste en una bipirámide con dos átomos de Pt en caras opuestas, pero en su optimización esta geometría inicial se pierde y adquiere la geometría que se muestra en la Figura 5.2.1.1 (isómero I). El resto de las estructuras optimizadas se conservan prácticamente sin cambios su geometría inicial. A excepción de la estructura con geometría plana, que pierde su configuración inicial y adquiere ligeramente geometría tridimensional después de su optimización, como el resto de las estructuras. Todas las geometrías se optimizaron para las multiplicidades de espín $1,3,5$ y 7.

En la Figura 5.2.1.1 se muestran las geométricas optimizadas de platino con la multiplicidad de espín que dieron lugar a la estructura más estable. El isómero de menor energía se presenta en "isómero I" para multiplicidad de espín triplete. Después de los isómeros IV y VI que no presenta ningún elemento de simetría, esta si presenta al menos uno, un plano de simetría que pasa por los átomos 1, 2, 4, y 8 y que la divide en dos partes iguales (Figura 5.2.2.2). Su energía de enlace (BE), fue la más baja de todas, por lo cual se tomó como el fundamental de la nanopartícula de $\mathrm{Pt}_{8}$, para hacer estudio de la adsorción con la molécula de óxido nitroso. 


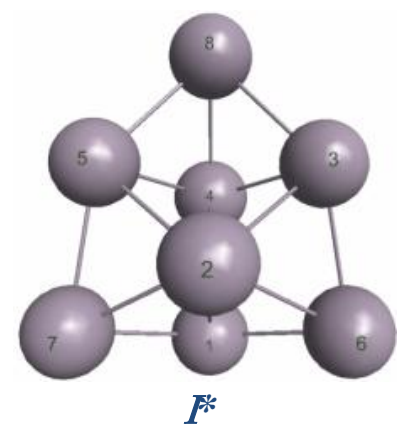

$M=3$

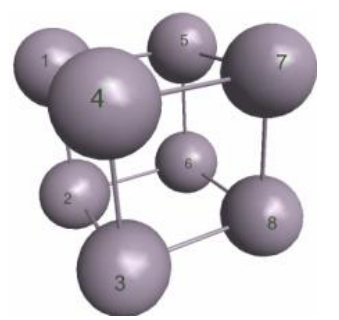

III

$M=7$

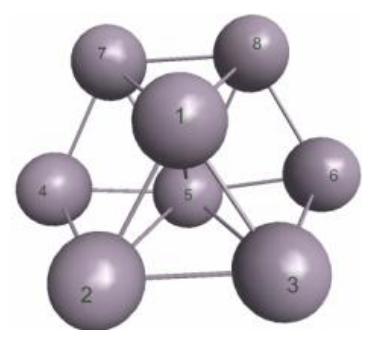

$\boldsymbol{V}$

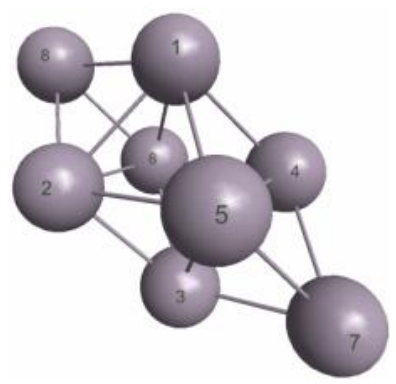

II

$M=7$

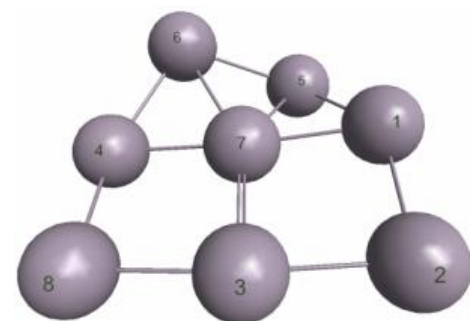

N

$M=5$

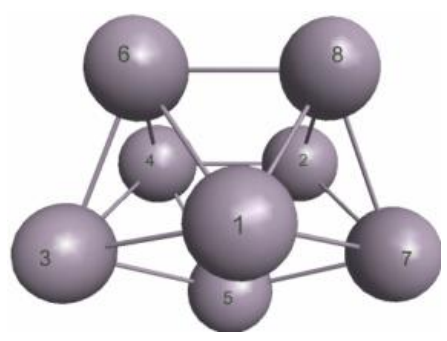

VI

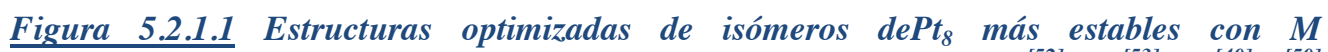
correspondiente. *Propuesta en este trabajo y $[56], I^{[52]}, I I I^{[53]}, I V^{[40]}, V^{[50]}$ $y V I^{[54]}$.

En la Tabla 5.2.1.1 se encuentran los parámetros energéticos determinados para cada una de estas estructuras optimizadas. Este estado (isómero I) el de menor energía, no está degenerado $(\mathrm{EB}>3 \mathrm{kcal} / \mathrm{mol})$ con los obtenidos al optimizar el resto de estructuras. En cambio estas otras geometrías suelen presentar estados degenerados, aunque no todas.

Por ejemplo la estructura de isómero II (Figura 5.2.1.1), tiene dos estados de multiplicidad de espín 5 y 7 con energías cercanas, menos de $3 \mathrm{kcal} / \mathrm{mol}$, la estructura 
geométrica de III, posee estados de multiplicidad de espín 3 y 7 con energías similares, menos de $1 \mathrm{kcal} / \mathrm{mol}$, y la geometría de VI, también con los estados de M 5 y 7 presenta energías casi iguales $(<1 \mathrm{kcal} / \mathrm{mol})$. El estado más cercano al fundamental (isómero I en M 3) es el de la estructura IV con $\mathrm{BE}=-645.55 \mathrm{kcal} / \mathrm{mol}$, la energía del fundamental es $653.18 \mathrm{kcal} / \mathrm{mol}$, su energía relativa es $7.63 \mathrm{kcal} / \mathrm{mol}$.

Tabla 5.2.1.1 Parámetros energéticos de estructuras geométricas de Pt. Multiplicidad de espín (M), energías de enlace $(B E)$ y energías relativas $\left(E_{\text {relativa }}\right)$ de los seis isómeros de platino.

\begin{tabular}{|c|c|c|c|}
\hline $\begin{array}{l}\text { Estructura } \\
\text { Geométrica }\end{array}$ & $\begin{array}{c}M \\
2 s+1\end{array}$ & $\begin{array}{c}\text { BE } \\
\mathrm{kcal} / \mathrm{mol}\end{array}$ & $\begin{array}{c}E_{\text {relativa }} \\
\text { kcal/mol }\end{array}$ \\
\hline \multirow{4}{*}{ I } & 1 & -640.75 & 12.43 \\
\hline & 3 & -653.18 & 0.00 \\
\hline & 5 & -644.14 & 9.04 \\
\hline & 7 & -642.75 & 10.43 \\
\hline \multirow{4}{*}{ II } & 1 & -571.59 & 81.59 \\
\hline & 3 & --- & $*$ \\
\hline & 5 & -631.31 & 21.87 \\
\hline & 7 & -633.68 & 19.50 \\
\hline \multirow{4}{*}{ III } & 1 & -624.89 & 28.29 \\
\hline & 3 & -636.95 & 16.23 \\
\hline & 5 & -636.04 & 17.14 \\
\hline & 7 & -639.33 & 13.85 \\
\hline \multirow{4}{*}{ IV } & 1 & -637.01 & 16.17 \\
\hline & 3 & -638.36 & 14.82 \\
\hline & 5 & -645.55 & 7.63 \\
\hline & 7 & -636.96 & 16.22 \\
\hline \multirow{4}{*}{ V } & 1 & -618.41 & 34.77 \\
\hline & 3 & -636.29 & 16.89 \\
\hline & 5 & -641.51 & 11.67 \\
\hline & 7 & --- & $*$ \\
\hline \multirow{4}{*}{ VI } & 1 & -630.10 & 23.08 \\
\hline & 3 & -633.25 & 19.93 \\
\hline & 5 & -635.90 & 17.28 \\
\hline & 7 & -635.33 & 17.85 \\
\hline
\end{tabular}

* Problemas de convergencia, no se muestran resultados. 
En la Tabla 5.2.1.2 se muestran los principales parámetros geométricos determinados para los 6 isómeros de $\mathrm{Pt}_{8}$. Las distancia promedio Pt-Pt reportadas en la literatura van de 2.52 a $2.72 \AA$. El del "isómero I" en este trabajo está en ese intervalo, $2.61 \pm 0.12 \AA$. En el resto de estructuras estudiadas esta distancia está entre 2.50 y $2.66 \AA$.

Tabla 5.2.1.2 Parámetros geométricos de estructuras optimizadas de Pt. Multiplicidad (M), frecuencias de vibración (v) y distancias de enlaces promedio (d).

\begin{tabular}{|c|c|c|c|c|}
\hline $\begin{array}{l}\text { Estructura } \\
\text { geométrica }\end{array}$ & $\begin{array}{c}M \\
2 s+1\end{array}$ & $\begin{array}{l}v^{P t-P t} \\
c m^{-1}\end{array}$ & $\begin{array}{c}\boldsymbol{d}_{P t-P t} \\
\AA\end{array}$ & $\begin{array}{c} \pm d_{P t-P t} \\
\stackrel{\circ}{\AA}\end{array}$ \\
\hline \multirow{4}{*}{ I } & 1 & 210 & 2.61 & 0.10 \\
\hline & 3 & 195 & 2.61 & 0.12 \\
\hline & 5 & 203 & 2.63 & 0.09 \\
\hline & 7 & 199 & 2.65 & 0.09 \\
\hline \multirow{4}{*}{ II } & 1 & 218 & 2.65 & 0.02 \\
\hline & 3 & --- & --- & $*$ \\
\hline & 5 & 182 & 2.65 & 0.03 \\
\hline & 7 & 137 & 2.65 & 0.02 \\
\hline \multirow{4}{*}{ III } & 1 & 171 & 2.50 & 0.00 \\
\hline & 3 & 171 & 2.52 & 0.01 \\
\hline & 5 & 174 & 2.51 & 0.00 \\
\hline & 7 & 163 & 2.52 & 0.00 \\
\hline \multirow{4}{*}{ IV } & 1 & 226 & 2.55 & 0.07 \\
\hline & 3 & 222 & 2.55 & 0.07 \\
\hline & 5 & 226 & 2.51 & 0.06 \\
\hline & 7 & 211 & 2.55 & 0.07 \\
\hline \multirow{4}{*}{ V } & 1 & 201 & 2.64 & 0.10 \\
\hline & 3 & 196 & 2.64 & 0.10 \\
\hline & 5 & 197 & 2.65 & 0.13 \\
\hline & 7 & --- & --- & $*$ \\
\hline \multirow{4}{*}{ VI } & 1 & 192 & 2.65 & 0.04 \\
\hline & 3 & 144 & 2.65 & 0.04 \\
\hline & 5 & 147 & 2.63 & 0.07 \\
\hline & 7 & 180 & 2.66 & 0.07 \\
\hline
\end{tabular}

*Problemas de convergencia, no se muestran resultados. 
Las frecuencias de vibración son bajas, como es de esperar en un cúmulo de átomos pesados. En algunas estructuras geométricas los átomos se unen a distancias menores y por lo tanto sus vibraciones se dificultan, por ejemplo en el "isómero III" la distancia media es de $2.50 \AA$ y su frecuencia de vibración es una de las más bajas, $171.4 \mathrm{~cm}^{-1}$. Por supuesto también influye la forma en que se unen los átomos.

En la siguiente figura 5.2.1.2 se muestra la geometría y distancias de enlace del isómero I, con diferentes ángulos de visualización.

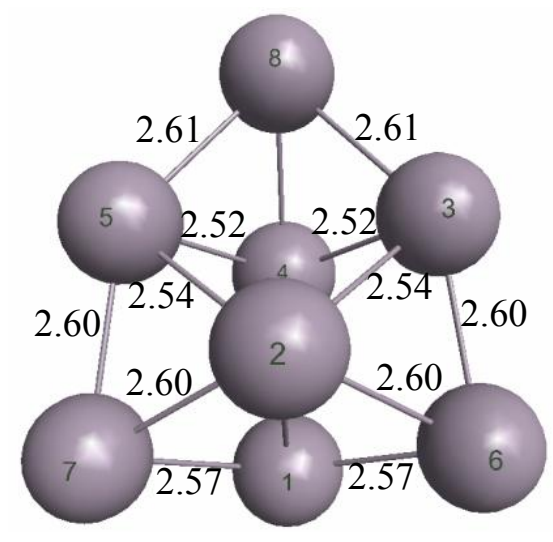

a)

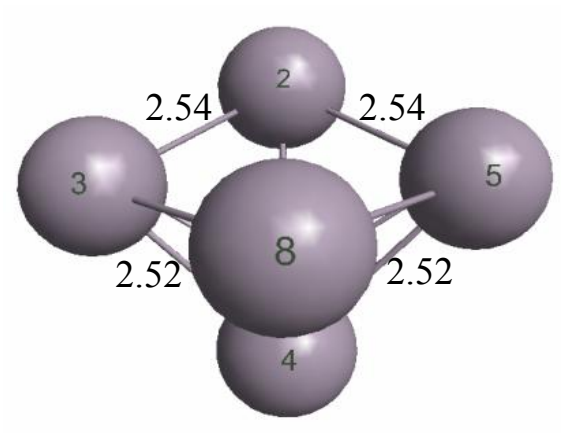

c)

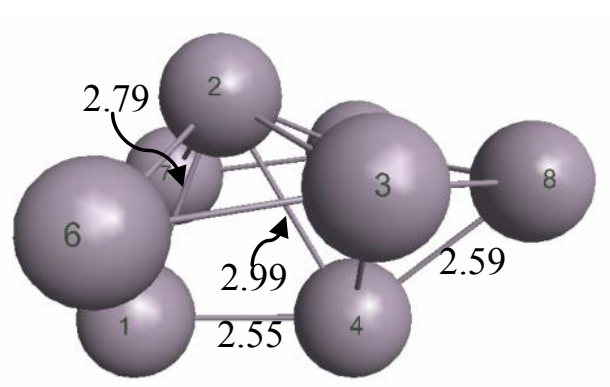

b)

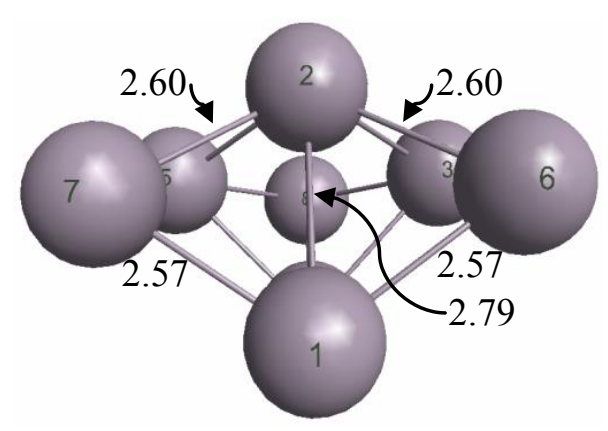

d)

Figura 5.2.1.2 Distancias de enlace y diferentes vistas de geometría del isómero I: a) vista desde arriba, b) vista lateral izquierda, c) vista frontal y d) vista desde atrás.

Como se mencionó anteriormente el isómero I, el de menor energía presenta un elemento de simetría en su estructura, este elemento consiste en un plano de simetría 
que divide su geometría en dos partes iguales, este plano pasa por los átomos 1, 2, 4 y 8 como se muestra en (a) en la Figura 5.2.1.3. Lo que hace que la nanopartícula presente sitios equivalentes, como por ejemplo entre átomos, 6 con el 7 y el 3 con el 5, un análisis de cargas Hirshfeld también demuestran su equivalencia como se puede ver en (b) de la Figura 5.2.1.3

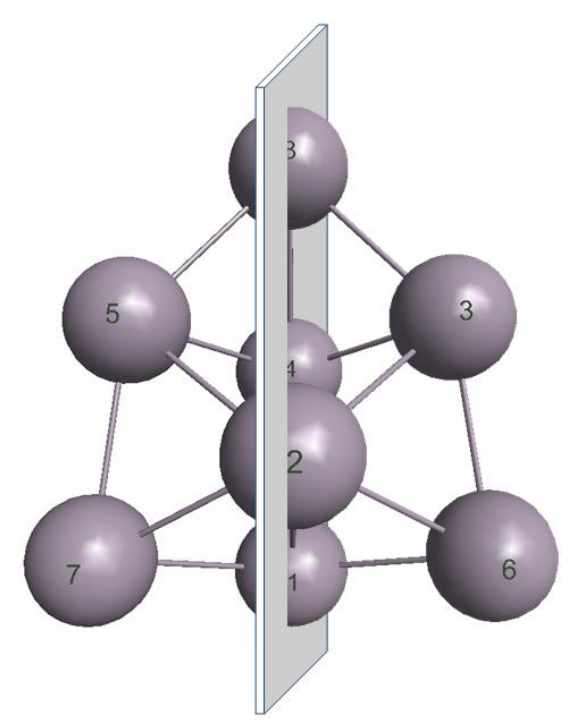

a)

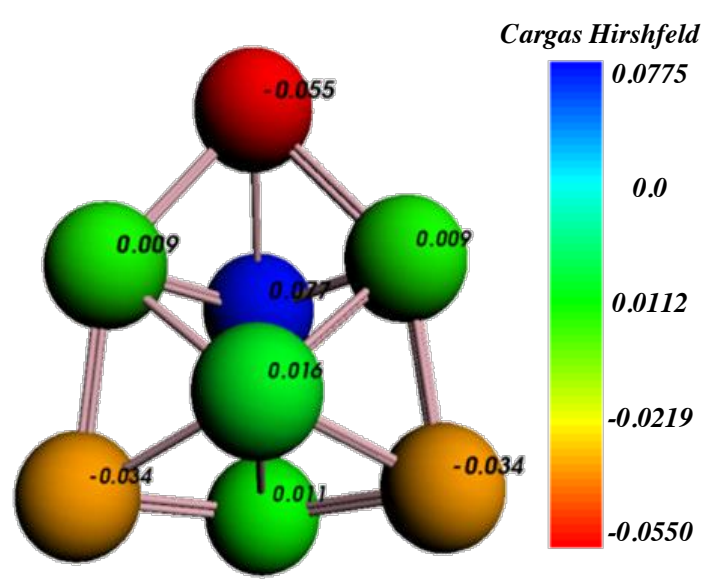

b)

Figura 5.2.1.3 a) Plano de simetría formado por los átomos 1, 2, 4 y 8, divide geometría de la geometría de la nanopartícula de ${ }^{3} \mathrm{Pt}_{8}$ (Isómero I) en dos partes iguales. b) Distribución de cargas Hirshfeld del Isómero I, ${ }^{3} \mathrm{Pt}_{8}$ (regiones en rojo ricas en e- $y$ regiones en azul deficientes).

Otros sitios están formados por enlaces entre los átomos 5-7 equivalente con 36, 2-7 con 2-6, 2-3 con 2-5, 4-5 con 4-3, 5-8 con 3-8 así como 1-7 con 1-6. Finalmente equivalencia entre caras formadas por los átomos 2-5-7 con 2-3-6 y 4-5-8 con 3-4-8. La presencia de estos sitios en la geometría de la nanopartícula disminuye el número de interacciones del $\mathrm{N}_{2} \mathrm{O}$ en este isómero I.

A continuación se realizara el estudio de las adsorciones de la molécula del $\mathrm{N}_{2} \mathrm{O}$ sobre diferentes sitios del isómero I (isómero con la geometría más estable) para determinar su reactividad frente a la disociación del óxido. 


\subsubsection{Estudio de la disociación del $\mathrm{N}_{2} \mathrm{O}$ sobre $\mathrm{Pt}_{8}$. Identificación de sitios de activación por $\mathrm{N}_{2} \mathrm{O}$}

En esta sección se estudia la adsorción de la molécula del $\left({ }^{1} \mathrm{~N}_{2} \mathrm{O}\right)$ sobre la nanopartícula de platino $\left({ }^{3} \mathrm{Pt}_{8}\right)$, Los acercamientos del $\mathrm{N}_{2} \mathrm{O}$ hacia la nanopartícula de platino se dividió en tres etapas, la 1era consistió en aproximar la molécula hacia los átomos de Pt directamente. En la 2da la molécula se acercó hacia enlaces Pt-Pt y finalmente la interacción hacia las caras. Los acercamientos se realizaron en todos los posibles sitios reactivos excepto los que tienen equivalencia. Las características de la interacción se describen en cada uno de los apartados.

\section{I. $\quad$ Interacción directa sobre átomos del platino.}

Para este tipo de interacción se examinaron tres acercamientos diferentes a un átomo de Pt de la nanopartícula Figura 5.2.2.1, en el 1ero, el $\mathrm{N}_{2} \mathrm{O}$ se aproxima de manera lineal por el lado del átomo de $\mathrm{O}$ al átomo de Pt inciso (a). En la segunda el $\mathrm{N}_{2} \mathrm{O}$ se aproxima de manera lineal por el lado del átomo de $\mathrm{N}$ al átomo de Pt inciso (b) y finalmente la molécula del óxido se aproxima perpendicular con el átomo de $\mathrm{N}$ intermedio hacia al átomo de Pt inciso (c). Las distancias iníciales de interacción entre ambos es $3 \AA$.

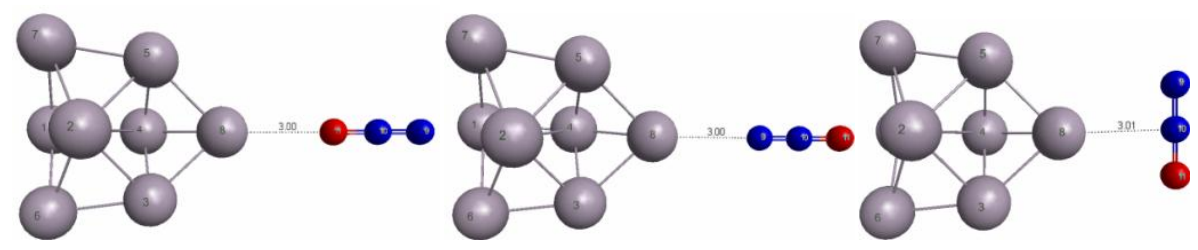

a)

b)

c)

Figura 5.2.2.1 Aproximaciones de la molécula de $\mathrm{N}_{2} \mathrm{O}$ hacia el isómero $\mathrm{I}$, a) lineal al átomo de Pt por el oxígeno, b) lineal por el $N$ terminal y c) el $N$ intermedio al átomo de Pt, perpendicular al eje de simetría de $\mathrm{Pt}_{8}$. 
En la Figura 5.2.2.2 se muestran acercamientos favorables para la disociación de la molécula de $\mathrm{N}_{2} \mathrm{O}$ así como también su valor de multiplicidad de espín (M). En el resto de las interacciones no favorables los resultados se muestran en el Anexo 9.1.5

Las interacciones se etiquetaron de la siguiente manera: Ej. Para la interacción $\underline{\text { I- }}$ $\underline{\mathrm{A} 8 \mathrm{O}}$, ler termino "I" por isómero I, 2do termino "A" interacción en átomos del cúmulo de $\mathrm{Pt}$, 3er termino "8" átomo ó sitio de interacción y ultimo termino "O" átomo del $\mathrm{N}_{2} \mathrm{O}$ que se acerca al cúmulo de $\mathrm{Pt}$.

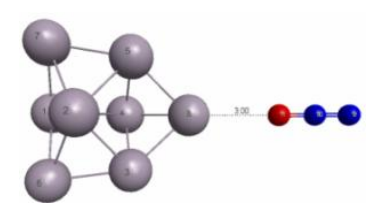

I-A80

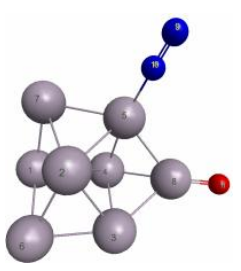

$\mathbf{M}=1$

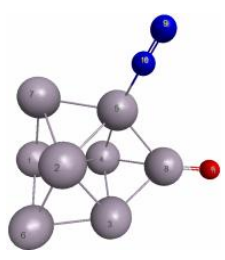

$\mathbf{M}=3$

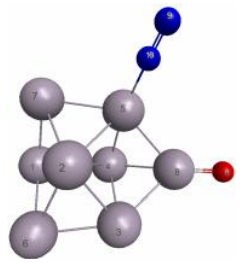

$\mathbf{M}=3$

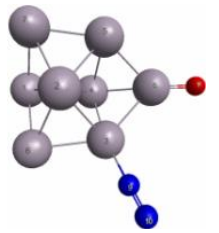

$\mathrm{M}=5$

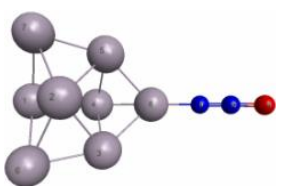

$\mathbf{M}=5$
$*$

$\mathrm{M}=7$

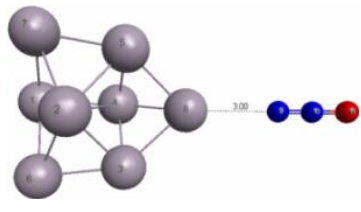

I-A8N1
$\mathbf{M}=1$
$*$

Figura 5.2.2.2 Geometrías iníciales de interacción del $\mathrm{N}_{2} \mathrm{O}$ hacia isómero I, a) I-A8O por el átomo de $O$ y b) I-A8N1 por el átomo de $N$ terminal, con diferentes multiplicidades de espín $(M=2 s+1) .\left({ }^{*}\right)$ No hay resultados, problemas de convergencia con el cálculo.

Descripción de la reacción en el sitio activo Figura 5.2.2.2

I-A80: $\mathrm{El}$ átomo de $\mathrm{O}$ del $\mathrm{N}_{2} \mathrm{O}$ interacciona directamente con el átomo 8 del isómero I, obteniendo disociación del enlace $\mathrm{N}-\mathrm{O}$, esto ocurre para multiplicidad de espín 1, 3 y 5. Las energías de disociación son: -50.81, 52.77 y $-62.48 \mathrm{kcal} / \mathrm{mol}$ respectivamente. Ver Figura 5.2.2.2 
I-A8N1: $\mathrm{El}$ átomo de $\mathrm{N}$ terminal del $\mathrm{N}_{2} \mathrm{O}$ interacciona directamente con el sitio 8 del isómero I, se obtiene disociación del enlace N-O con energía de $62.51 \mathrm{kcal} / \mathrm{mol}$ solo para multiplicidad de espín 3 , y adsorción sin disociar para multiplicidad 5 y 7, para estas últimas dos multiplicidades de espín la molécula de $\mathrm{N}_{2} \mathrm{O}$ se adsorbe en el átomo de interacción inicial de Pt por el $\mathrm{N}$ terminal del $\mathrm{N}_{2} \mathrm{O}$. Como se muestra en la Figura 5.2.2.2

En la Tabla 5.2.2.1 se resumen los parámetros de energía con acercamientos favorables a la reducción de la molécula de $\mathrm{N}_{2} \mathrm{O}$ y su valor de multiplicidad de espín (M) correspondiente. La interacción de más baja energía se obtiene para el acercamiento por el átomo de $\mathrm{N}$ terminal de la molécula de $\mathrm{N}_{2} \mathrm{O}$ hacia el sitio 8 del isómero I (I-A8N1), con multiplicidad de espín 3, otra interacción de energía muy similar está a $0.03 \mathrm{kcal} / \mathrm{mol}$ por arriba este, este es I-A8O con multiplicidad de espín 5 . El resto de las interacciones no favorables se resumen en el anexo 9.1.5

Tabla 5.2.2.1 Parámetros energéticos de la interacción de $\mathrm{N}_{2} \mathrm{O}$ hacia átomos del isómero I. Energías de disociación $\left(E_{\text {dis }}\right)$ y energía relativa $\left(E_{\text {relativa }}\right)$ respecto al estado de más baja energía.

\begin{tabular}{ccccc}
\hline \hline $\begin{array}{c}\text { Tipo de } \\
\text { interacción }\end{array}$ & $\boldsymbol{M}$ & $\begin{array}{c}\boldsymbol{E}_{\text {dis }} \\
\boldsymbol{k c a l / m o l}\end{array}$ & $\begin{array}{c}\boldsymbol{E}_{\text {relativa }} \\
\boldsymbol{k c a l / m o l}\end{array}$ & $\begin{array}{c}\text { Enlace } \\
\text { /disociación }\end{array}$ \\
\hline \hline & 1 & -50.81 & 11.7 & $\mathrm{si} / \mathrm{si}$ \\
I-A8O & 3 & -52.77 & 9.74 & $\mathrm{si} / \mathrm{si}$ \\
& 5 & -62.48 & 0.03 & $\mathrm{si} / \mathrm{si}$ \\
$*$ \\
\hline
\end{tabular}

* No hay resultados, problemas de convergencia con el cálculo.

En la Tabla 5.2.2.2 se resumen los parámetros geométricos de acercamientos favorables en la disociación del enlace N-O y su valor de multiplicidad de espín (M). 
Para la interacción de más baja energía, I-A8N1 (M 3), los parámetros que se

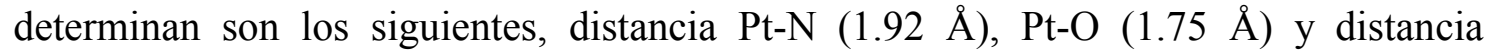
promedio Pt-Pt $(2.63 \pm 0.12 \AA)$, el átomo de $\mathrm{O}$ forma el enlace más fuerte con el átomo del metal al finalizar la reacción.

Las frecuencias de vibración que se reportan son todas reales y máximas, su valor corresponde a un modo normal de vibración, estiramiento del enlace $\mathrm{N}-\mathrm{N}$, su valor más grande se presenta cuando hay disociación y el más bajo cuando solo hay adsorción sin disociar.

En el resto de los casos donde se determinó la reducción de este óxido los parámetros geométricos que se obtienen son muy cercanos o casi iguales a los obtenidos para el sistema de más baja energía.

Tabla 5.2.2.2 Parámetros geométricos de la interacción de $\mathrm{N}_{2} \mathrm{O}$ hacia átomos del isómero I. Multiplicidad de espín (M), frecuencias de vibración (v) y distancias de enlace (d).

\begin{tabular}{|c|c|c|c|c|c|c|}
\hline $\begin{array}{c}\text { Tipo de } \\
\text { interacción }\end{array}$ & $\begin{array}{c}M \\
2 s+1\end{array}$ & $\begin{array}{l}v^{N-N} \\
\mathrm{~cm}^{-1}\end{array}$ & $\begin{array}{c}\mathbf{d}_{\text {Pt-O }} \\
\AA\end{array}$ & $\begin{array}{c}\mathbf{d}_{\mathrm{Pt}-\mathrm{N}} \\
\AA\end{array}$ & $\begin{array}{c}\mathbf{d}_{\mathbf{P t}-\mathbf{P t}} \\
\AA\end{array}$ & $\begin{array}{c} \pm \mathbf{d}_{\mathbf{P t}-\mathrm{Pt}} \\
\stackrel{\AA}{\mathbf{A}}\end{array}$ \\
\hline \multirow{4}{*}{ I-A80 } & 1 & 2169 & 1.75 & 1.92 & 2.62 & 0.10 \\
\hline & 3 & 2169 & 1.75 & 1.92 & 2.62 & 0.10 \\
\hline & 5 & 2167 & 1.75 & 1.92 & 2.63 & 0.12 \\
\hline & 7 & --- & --- & --- & --- & * \\
\hline \multirow{4}{*}{ I-A8N1 } & 1 & --- & --- & --- & --- & $*$ \\
\hline & 3 & 2168 & 1.75 & 1.92 & 2.63 & 0.12 \\
\hline & 5 & 1256 & - & 1.96 & 2.59 & 0.08 \\
\hline & 7 & 1254 & - & 1.94 & 2.59 & 0.08 \\
\hline
\end{tabular}

* No hay resultados, problemas de convergencia con el cálculo.

Los productos de la reducción se adsorben sobre la superficie del isómero I, el átomo de $\mathrm{O}$ queda adsorbido sobre el sitio de interacción (sitio 8) de la nanopartícula y la molécula de $\mathrm{N}_{2}$ en un átomo vecino inmediato al de interacción. 
Algunas de las características que presenta este sitio (átomo 8) con respecto al resto de los átomos del isómero I: se concentra la mayor cantidad de carga favoreciendo su reactividad frente a la reducción del $\mathrm{N}_{2} \mathrm{O}$ (b) de la Figura 5.1.2.1, hay mayor número de electrones disponibles para ser transferidos hacia la molécula del óxido. Otro factor importante es el estado electrónico del metal ya que la reducción del óxido se favoreció generalmente para las multiplicidades de espín de baja y alta energía (M 1, 3 y 5). De esta manera el metal ofrece mayor número de electrones disponibles en sus orbitales para participar en la reacción.

Acercamientos a otros sitios del isómero I no resultan favorables en la reducción del $\mathrm{N}_{2} \mathrm{O}$. A diferencia del sitio 8 que si presento reactividad el resto no posee suficiente carga para ser promovida del metal hacia el óxido y generar la disociación del enlace $\mathrm{N}-\mathrm{O}$, esta solo es suficiente para formar el enlace metal- $\mathrm{N}_{2} \mathrm{O}$.

A continuación se hace el estudio de las adsorciones del $\mathrm{N}_{2} \mathrm{O}$ hacia sitios generados por enlaces del isómero I, para determinar su reactividad para la disociación del óxido.

\section{I. $\quad$ Interacción sobre enlaces platino-platino}

En este tipo de interacción la molécula de $\mathrm{N}_{2} \mathrm{O}$ se acercó directamente a enlaces

Pt-Pt del isómero I, por tres formas geométricas diferentes (Figura 5.2.2.3): paralelo al enlace Pt-Pt (a), perpendicular por el átomo de $\mathrm{O}(\mathrm{b})$, perpendicular por $\mathrm{N}$ terminal (c) y perpendicular por el átomo de $\mathrm{N}$ intermedio (d). Las distancias iníciales de interacción son de $2.5 \AA$. 


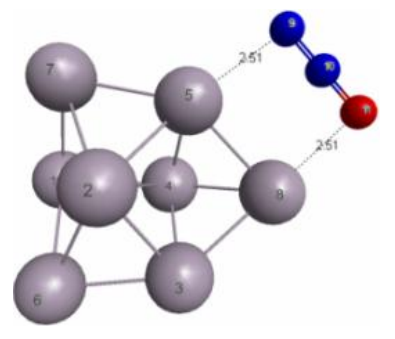

a)

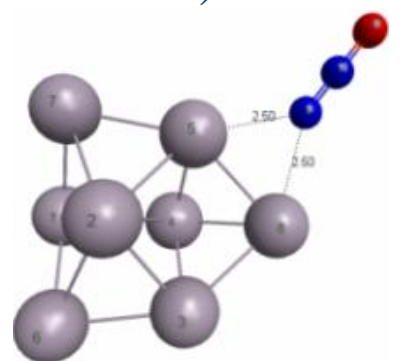

c)

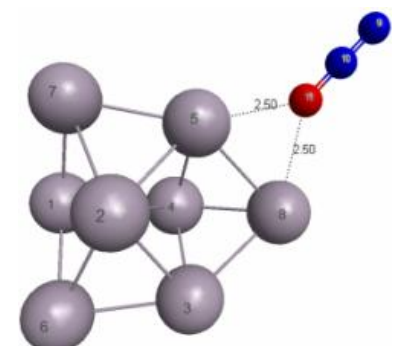

b)

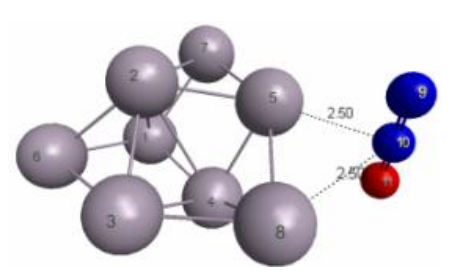

d)

Figura 5.2.2.3 Acercamientos del $\mathrm{N}_{2} \mathrm{O}$ hacia el isómero I, a) paralelo a dos átomos de $P t, b)$ perpendicular al enlace de Pt por el átomo de $O$, c) perpendicular al enlace de Pt por el átomo de $N$ terminal y d) perpendicular al enlace de Pt por el átomo de $\mathrm{N}$ intermedio.

De estos cuatro diferentes acercamientos de la molécula del $\mathrm{N}_{2} \mathrm{O}$ hacia el isómero I, solo cuando el acercamiento del óxido es paralelo (a) de Figura 5.2.2.3 hacia la superficie se favorece la disociación de la molécula del óxido.

En la Figura 5.2.2.4 se muestran 5 interacciones que favorecen la disociación de la molécula $\mathrm{N}_{2} \mathrm{O}$, con su valor de multiplicidad de espín (M) cuatro de estas interacciones tienen sitios equivalentes como se mencionó en la sección 5.2.2, se detallan más adelante.

Las interacciones se etiquetaron de la siguiente manera: Ej. Para la interacción $\underline{\text { I-E58, }}$ 1er termino "I" por isómero I, 2do termino "E" por interacción hacia enlaces Pt-Pt del cúmulo y últimos dos términos "58" representa el enlace ó sitio de interacción. 


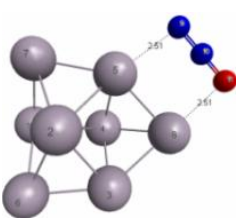

I-E58

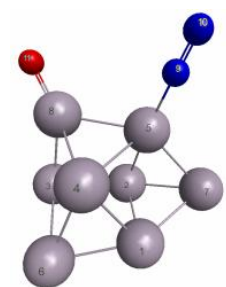

$M=1$

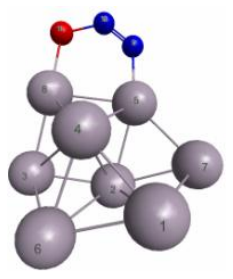

$\mathrm{M}=3$

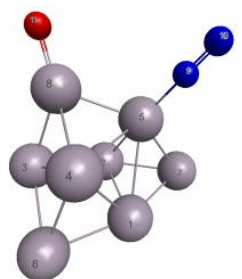

$M=5$

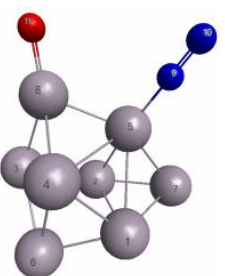

$\mathrm{M}=7$

I-E38**

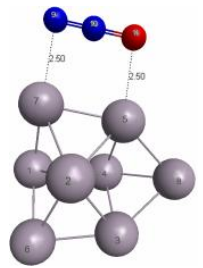

I-E57

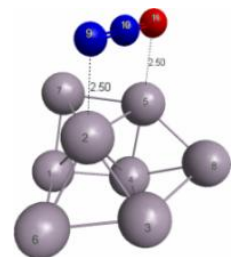

I-E25

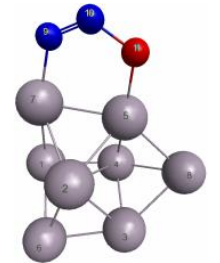

$\mathrm{M}=1$

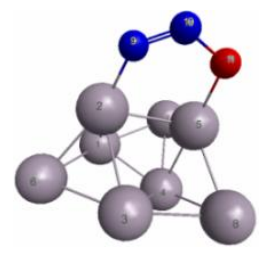

$\mathrm{M}=1$

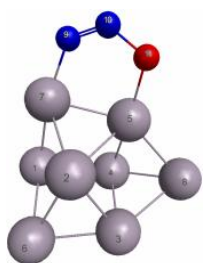

$\mathrm{M}=3$

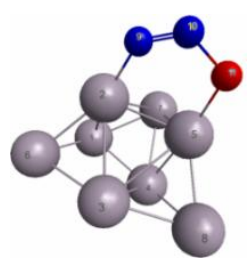

$\mathrm{M}=3$

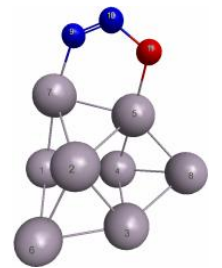

$M=5$

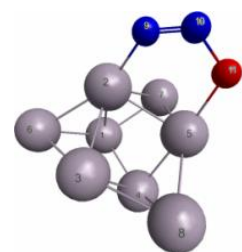

$M=5$

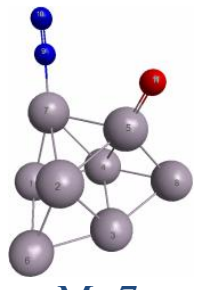

$M=7$

I-E23**

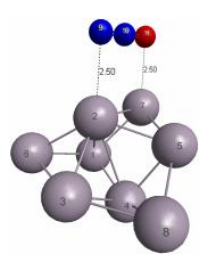

I-E27

I-E26**

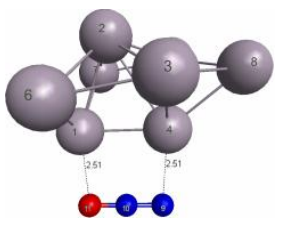

I-E14

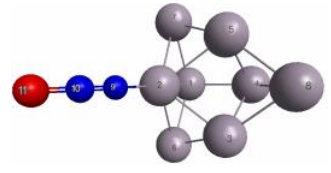

$$
\mathrm{M}=1
$$

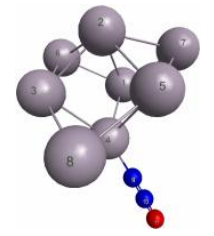

$\mathrm{M}=1$
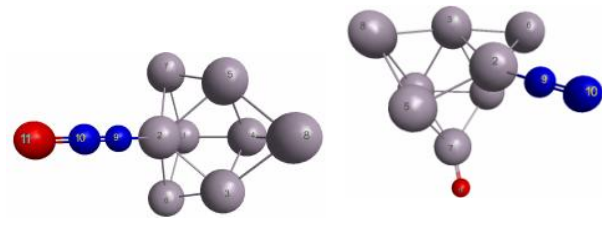

$M=5$
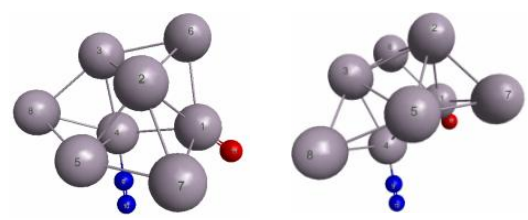

$M=5$

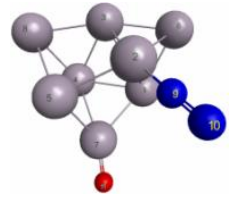

$M=7$

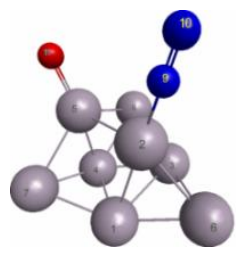

$M=7$

Figura 5.2.2.4 Geometrías iníciales de interacción $\mathrm{Pt}_{8}+\mathrm{N}_{2} \mathrm{O}$ en diferentes enlaces Pt-Pt del isómero-I $y$ sus resultados con distintas multiplicidades de espín, $M=2 s+1$. (**) Interacciones en sitios equivalentes al indicado, por simetría del isómero I, se generan estos sitios. 
Descripción de las interacciones del $\mathrm{N}_{2} \mathrm{O}$ hacia enlaces Pt-Pt, Figura 5.2.2.4

I-E58: La molécula de $\mathrm{N}_{2} \mathrm{O}$ se acercó paralela al enlace formado por los átomos 5-8 del isómero I. Obteniendo reducción de la molécula del óxido en multiplicidad de espín 1, 5 y 7, y absorción sin disociar en M 3 con los extremos de molécula del óxido unidos al isómero I. Las energías de disociación del enlace N-O son: $65.83,67.33,56.49 \mathrm{kcal} / \mathrm{mol}$ respectivamente. De manera equivalente a I-B58, la interacción I-B38 genera otro sitio equivalente con el $\mathrm{N}_{2} \mathrm{O}$ con las mismas características.

I-E57: La molécula de $\mathrm{N}_{2} \mathrm{O}$ interacciona paralela al enlace Pt-Pt, formado átomos 5-7 del isómero I. Obteniendo reducción del óxido solo en multiplicidad de espín 7 con energía de $-53.28 \mathrm{kcal} / \mathrm{mol}$, en multiplicidad de espín 1,3 y 5 solo se presentó adsorción sin disociar. El $\mathrm{N}_{2} \mathrm{O}$ se enlaza por sus extremos a la superficie del isómero I, formando un enlace tipo puenteado. También equivalente a I-B57, la interacción del $\mathrm{N}_{2} \mathrm{O}$ con el enlace 3-6 presenta el mismo comportamiento.

I-E25: Acercando la molécula de $\mathrm{N}_{2} \mathrm{O}$ paralela al enlace Pt-Pt formado átomos 2-5, se obtiene reducción del óxido en multiplicidad de espín 7 con energía de disociación de $-54.48 \mathrm{kcal} / \mathrm{mol} \mathrm{y}$ absorción sin disociar en multiplicidad de espín 1, 3 y 5. Formando un enlace tipo puenteado donde la molécula de $\mathrm{N}_{2} \mathrm{O}$ se enlaza al isómero I por sus extremos. De manera equivalente con los átomos 2-3 (por simetría de la nanopartícula) ocurre el mismo comportamiento con la molécula de óxido. I-B25 y I-B23 generan sitios reactivos equivalentes.

I-E27: Acercando la molécula de $\mathrm{N}_{2} \mathrm{O}$ paralela al enlace formado por átomos 1-7 del isómero I. Se obtiene rompimiento del enlace $\mathrm{N}-\mathrm{O}$ en multiplicidad de espín 5, 7 con energía de disociación de -68.67 y $-60.41 \mathrm{kcal} / \mathrm{mol}$. En M 1 y 3 solo se obtiene adsorción sin disociar, con la molécula de $\mathrm{N}_{2} \mathrm{O}$ enlazándose al isómero I por el $\mathrm{N}$ terminal. De manera similar I-B27 y I-B26 generan sitios reactivos equivalentes. 
I-E14: La molécula de $\mathrm{N}_{2} \mathrm{O}$ se aproxima paralela al enlace Pt-Pt formado por los átomos 1-4 del isómero I. Obteniendo rompimiento del enlace $\mathrm{N}-\mathrm{O}$ en multiplicidad de espín 5, 7 con una energía de disociación de -58.18 y -54.48 $\mathrm{kcal} / \mathrm{mol}$, en multiplicidad 1 y 3 solo se obtiene adsorción sin disociar, con el $\mathrm{N}_{2} \mathrm{O}$ enlazado a un átomo del isómero I de Pt por el $\mathrm{N}$ terminal.

La interacción de más baja energía se obtiene para, I-E27 (I-E57**), con multiplicidad de espín 5. Las interacciones con el valor más cercano a este con 1.34 y $2.84 \mathrm{kcal} / \mathrm{mol}$ es para la interacción: I-E58 (I-E38**) con multiplicidad de espín 5 y 1 respectivamente. La multiplicidad que se favorecen son las de más alta energía, 5 y 7. De esta manera se promueve una mayor cantidad de electrones disponibles en el metal, para ser transferidos a la molécula del $\mathrm{N}_{2} \mathrm{O}$ y así promover el rompimiento del enlace $\mathrm{O}-\mathrm{N}$.

En la Tabla 5.2.2.3 se resumen los resultados energéticos de los acercamientos favorables en la reducción del óxido nitroso, con su valor de multiplicidad de espín correspondiente. El resto de las interacciones con otros sitios que no favorecen la disociación del $\mathrm{N}_{2} \mathrm{O}$ se muestran en el Anexo 9.1.5 
Tabla 5.2.2.3 Parámetros de energía de la interacción de $\mathrm{N}_{2} \mathrm{O}$ hacia enlaces del isómero I. Energías de disociación $\left(E_{\text {dis }}\right)$ y energía relativa $\left(E_{\text {relativa }}\right)$.

\begin{tabular}{ccccc}
\hline $\begin{array}{c}\text { Tipo de } \\
\text { interacción }\end{array}$ & $\begin{array}{c}\mathbf{M} \\
\mathbf{2 s + 1}\end{array}$ & $\begin{array}{c}\mathbf{E}_{\mathbf{d i s}} \\
\mathbf{k c a l} / \mathbf{m o l}\end{array}$ & $\begin{array}{c}\mathbf{E}_{\text {relativa }} \\
\mathbf{k c a l} / \mathbf{m o l}\end{array}$ & $\begin{array}{c}\text { Enlace } \\
\text { /disociación }\end{array}$ \\
\hline \hline \multirow{2}{*}{ I-E58 } & 1 & -65.83 & 2.84 & $\mathrm{si} / \mathrm{si}$ \\
& 3 & -19.53 & 49.13 & $\mathrm{si} / \mathrm{no}$ \\
& 5 & -67.33 & 1.34 & $\mathrm{si} / \mathrm{si}$ \\
I-E57 & 7 & -56.49 & 12.18 & $\mathrm{si} / \mathrm{si}$ \\
\hline \multirow{3}{*}{ I-E25 } & 1 & -21.80 & 46.87 & $\mathrm{si} / \mathrm{no}$ \\
& 3 & -21.27 & 47.40 & $\mathrm{si} / \mathrm{no}$ \\
& 5 & -20.14 & 48.53 & $\mathrm{si} / \mathrm{no}$ \\
& 7 & -53.28 & 15.39 & $\mathrm{si} / \mathrm{si}$ \\
\hline I-E27 & 1 & -21.73 & 46.94 & $\mathrm{si} / \mathrm{no}$ \\
& 3 & -19.50 & 49.17 & $\mathrm{si} / \mathrm{no}$ \\
& 5 & -15.84 & 52.83 & $\mathrm{si} / \mathrm{no}$ \\
& 7 & -54.48 & 14.19 & $\mathrm{si} / \mathrm{si}$ \\
\hline \hline & 1 & -13.62 & 55.04 & $\mathrm{si} / \mathrm{no}$ \\
& 3 & -17.85 & 50.82 & $\mathrm{si} / \mathrm{no}$ \\
& 5 & -68.67 & 0.00 & $\mathrm{si} / \mathrm{si}$ \\
& 7 & -60.41 & 8.26 & $\mathrm{si} / \mathrm{si}$ \\
\hline & 1 & -14.48 & 54.19 & $\mathrm{si} / \mathrm{no}$ \\
& 3 & -19.24 & 49.43 & $\mathrm{si} / \mathrm{no}$ \\
& 5 & -58.18 & 10.49 & $\mathrm{si} / \mathrm{si}$ \\
& 5 & -54.48 & 14.19 & $\mathrm{si} / \mathrm{si}$ \\
\hline
\end{tabular}

En la Tabla 5.2.2.4 se resumen los parámetros geométricos de las interacciones que se favorecieron, en la interacción de más baja energía las distancias Pt-N (1.92 A), Pt-O (1.76 ̊̊), esta ultima más corta formando el enlace más fuerte con el metal y la distancia promedio Pt-Pt $(\sim 2.60 \AA)$.

Los valores de las frecuencias de vibración son todas reales y su valor máximo, corresponde a un modo normal de vibración, estiramiento del enlace $\mathrm{N}-\mathrm{N}$, los valores depende del ambiente que este rodeando este enlace, $\sim 2170 \mathrm{~cm}^{-1}$ en la disociación, $\sim 1520 \mathrm{~cm}^{-1}$ ó menor, dependiendo del enlace que forma la molécula del $\mathrm{N}_{2} \mathrm{O}$ sobre el isómero I. 
Tabla 5.2.2.4 Parámetros geométricos de la interacción de $\mathrm{N}_{2} \mathrm{O}$ hacia enlaces del isómero I. Multiplicidad de espín (M), frecuencias de vibración (v) y distancias de enlace (d).

\begin{tabular}{|c|c|c|c|c|c|c|}
\hline $\begin{array}{c}\text { Tipo de } \\
\text { interacción }\end{array}$ & $\begin{array}{c}M \\
2 s+1\end{array}$ & $\begin{array}{l}v^{N-N} \\
\mathrm{~cm}^{-1}\end{array}$ & $\begin{array}{c}\mathbf{d}_{\text {Pt-O }} \\
\AA\end{array}$ & $\begin{array}{c}\mathbf{d}_{\text {Pt-N }} \\
\AA\end{array}$ & $\begin{array}{c}\mathbf{d}_{\mathbf{P t}-\mathbf{P t}} \\
\AA\end{array}$ & $\begin{array}{l} \pm \mathbf{d}_{\mathbf{P t}-\mathrm{Pt}} \\
\stackrel{\circ}{\mathbf{A}}\end{array}$ \\
\hline \multirow{4}{*}{ I-E58 } & 1 & 2170 & 1.76 & 1.92 & 2.59 & 0.08 \\
\hline & 3 & 1526 & 1.99 & 1.96 & 2.64 & 0.12 \\
\hline & 5 & 2172 & 1.76 & 1.92 & 2.62 & 0.10 \\
\hline & 7 & 2175 & 1.77 & 1.92 & 2.63 & 0.12 \\
\hline \multirow{4}{*}{ I-E57 } & 1 & 1476 & 2.02 & 1.97 & 2.61 & 0.11 \\
\hline & 3 & 1508 & 2.05 & 1.95 & 2.59 & 0.07 \\
\hline & 5 & 1512 & 2.04 & 1.94 & 2.59 & 0.07 \\
\hline & 7 & 2152 & 1.79 & 1.90 & 2.63 & 0.1 \\
\hline \multirow{4}{*}{ I-E25 } & 1 & 1467 & 2.00 & 1.9 & 2.59 & 0.08 \\
\hline & 3 & 1515 & 2.00 & 1.8 & 2.59 & 0.08 \\
\hline & 5 & 1470 & 2.04 & 1.94 & 2.59 & 0.08 \\
\hline & 7 & 2151 & 1.79 & 1.91 & 2.61 & 0.11 \\
\hline \multirow{4}{*}{ I-E27 } & 1 & 1256 & - & 1.95 & 2.59 & 0.08 \\
\hline & 3 & 1257 & - & 1.97 & 2.59 & 0.08 \\
\hline & 5 & 2144 & 1.76 & 1.9 & 2.61 & 0.11 \\
\hline & 7 & 2163 & 1.77 & 1.92 & 2.62 & 0.10 \\
\hline \multirow{4}{*}{ I-E14 } & 1 & 1242 & - & 1.97 & 2.59 & 0.08 \\
\hline & 3 & 1258 & - & 1.97 & 2.59 & 0.08 \\
\hline & 5 & 2182 & 1.80 & 1.93 & 2.59 & 0.08 \\
\hline & 7 & 2152 & 1.79 & 1.91 & 2.61 & 0.11 \\
\hline
\end{tabular}

Estos resultados se favorecieron principalmente hacia átomos con cargas de valores pequeños. La distribución de carga que posee la nanopartícula hace que los sitios reactivos en este caso sea mayor. Los valores de multiplicidad que favorecen el rompimiento del enlace $\mathrm{N}-\mathrm{O}$ son los de más alta energía 5 y 7. Ambos parámetros, multiplicidad de espín y carga, favorecen y promueven la transferencia de electrones del metal hacia la molécula del óxido para debilitar y romper el enlace N-O.

Como se mencionó anteriormente por simetría de la nanopartícula en las tablas de resultados solo se muestra una parte equivalente de las interacciones. En general los resultados corresponden a un total de 9 interacciones. Cuatro sitios generaron sus 
equivalentes y solo uno se genera del plano de simetría como se describió anteriormente en cada uno de los acercamientos.

Finalmente se hace la interacción del óxido hacia sitios ahora generados por caras en el isómero I, para determinar la reactividad en la disociación de la molécula del óxido.

\section{Interacción sobre las caras del platino.}

En esta sección se hace el estudio de las interacciones de la molécula del óxido nitroso sobre las caras formadas en isómero I. Tomando en cuenta solo caras formadas por tres átomos. La descripción de los modos de acercamiento de la molécula de $\mathrm{N}_{2} \mathrm{O}$ hacia estas en la nanopartícula se ilustra en la figura 5.2.2.5. Las distancias iníciales de interacción entre el isómero y el $\mathrm{N}_{2} \mathrm{O}$ son de $3 \AA$.

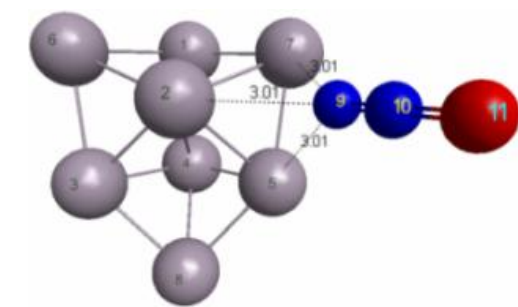

a)

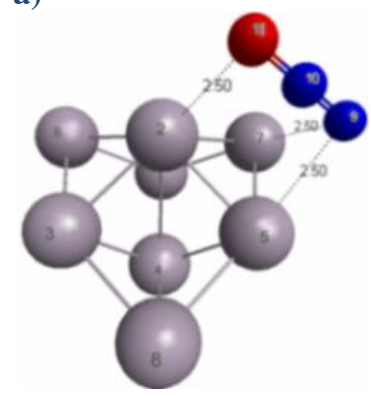

c)

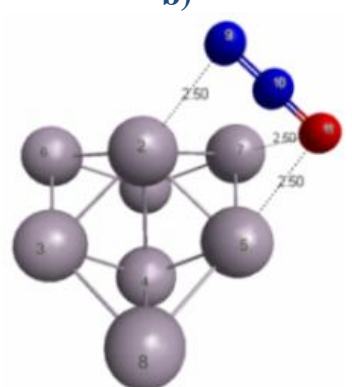

d)

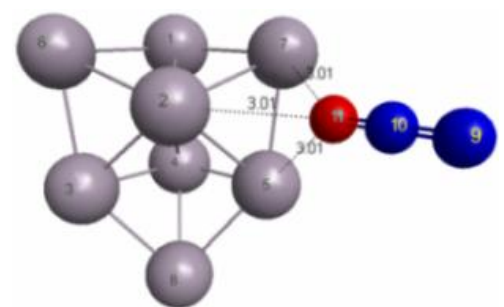

b)

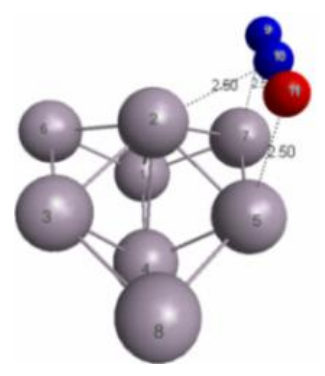

e)

Figura 5.2.2.5 Acercamientos de $\mathrm{N}_{2} \mathrm{O}$ hacia una cara del isómero I, a) perpendicular por el átomo de $N, b)$ perpendicular por el átomo de $\mathrm{O}, \mathrm{c}$ ) paralelo, interaccionando el átomo de $\mathrm{O}$ con un átomo de $\mathrm{Pt}$ del vértice, $d$ ) paralelo, interaccionando el átomo de $\mathrm{N}$ terminal con un átomo de $\mathrm{Pt}$ del vértice, e) paralelo a una cara, interaccionando con dos vértices de la cara. 
La interacción de la molécula de $\mathrm{N}_{2} \mathrm{O}$ hacia las caras del isómero I no da lugar a ningún sitio reactivo. Se obtienen solo adsorciones no disociativas de la molécula, en su mayoría quimisorciones. Contrario a los resultados reportados para $\mathrm{Rh}_{\mathrm{n}}(\mathrm{n}=2-4)$ [44], donde se obtiene reducción total de la molécula de $\mathrm{N}_{2} \mathrm{O}$, en este tipo de sitios usando la misma metodología.

El efecto de las cargas y la multiplicidad de espín no son suficiente para promover electrones del metal hacia la molécula de óxido, por lo que la reducción no se favorece para este tipo de sitios en isómero I. Resultados de estas interacción se reportan en Anexo 9.1.5

Los valores de las frecuencias de vibración (v) que se obtienen son todas reales, su valor máximo corresponde a una vibración característica de la molécula N-N, movimiento "stretching", en todos los casos.

En general todos los acercamientos probados del $\mathrm{N}_{2} \mathrm{O}$ hacia átomos, enlaces y caras hacia el isómero Ilos resultados favorables fueron aquellos donde el $\mathrm{N}_{2} \mathrm{O}$ se acerca paralelo a enlaces $\mathrm{Pt}-\mathrm{Pt}$, obteniendo el mayor número de casos de rompimiento de enlace $\mathrm{N}-\mathrm{O}$, estos sitios son preferibles por que se favorece la transferencia de carga de ambos átomos del metal hacia la molécula del $\mathrm{N}_{2} \mathrm{O}$, la reacción se desarrolla de manera espontánea en todo momento, $\mathrm{E}<0$. Los productos de la reacción en todos los casos estudiados se adsorben en los átomos iníciales de interacción y en átomos vecinos inmediatos a estos.

Además en esta estructura geométrica de $\mathrm{Pt}_{8}$ se determinó un sitio reactivo generado por un átomo de $\mathrm{Pt}$, aproximando el átomo de $\mathrm{O}$ del óxido directamente hacia el átomo del metal, en la literatura se reporta interacción por $\mathrm{N}$ terminar como favorable pero por oxígeno nunca, otros trabajos con $\mathrm{Rh}_{\mathrm{n}}(\mathrm{n}=2-4)$ [44] describen reducciones que se favorecen solo cuando la molécula de óxido se aproxima horizontal sobre una superficie o sobre un enlace. 


\subsubsection{Análisis de las cargas Hirshfeld de la reacción de $\mathrm{N}_{2} \mathrm{O}$ sobre $\mathrm{Pt}_{8}$}

Como se mencionó en la metodología, en el programa ADF existen tres diferentes aproximaciones para el análisis de distribución de carga electrónica. El esquema de Hirshfeld uno de los más empleados y confiables para determinar la distribución de carga en una molécula, porque se basa en el uso de densidades atómicas para la partición de la densidad electrónica molecular del sistema total en equilibrio, por lo cual en este trabajo se empleó este esquema para calcular la distribución de carga en cada sistema.

El análisis de las cargas de Hirshfeld en las interacciones de $\mathrm{N}_{2} \mathrm{O}$ con $\mathrm{Pt}_{8}$ antes y después de la interacción se realizó para todos los casos estudiados pero solo se reporta para casos favorables de rompimiento del enlace $\mathrm{N}-\mathrm{O}$ en la molécula del $\mathrm{N}_{2} \mathrm{O}$, como a continuación se realiza su análisis.

En la Figura 5.2.3.1 se resumen las interacciones que favorecieron la disociación de la molécula del $\mathrm{N}_{2} \mathrm{O}$ en acercamientos a un átomo de Pt (sitio reactivo), acercando por los extremos de la molécula de óxido a la superficie de la nanopartícula.

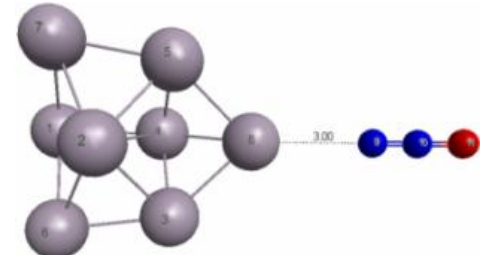

a) ${ }^{3} I-A 8 N 1$

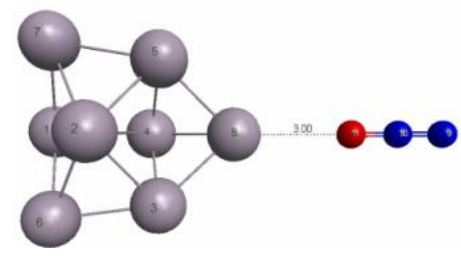

b) ${ }^{5} \mathrm{I}-\mathrm{A} 8 \mathrm{O}$

Figura 5.2.3.1 Acercamientos favorables a la disociación de la molécula del óxido. El sitio más reactivo esta en el átomo 8 en ambas interacciones, a)

${ }^{3} I-A 8 N 1$ y b) ${ }^{5} I-A 8 O$.

En la Tabla 5.2.3.1 se muestran los valores de las cargas iníciales $\left(q_{i}\right)$ antes de la interacción, cargas finales $\left(q_{f}\right)$ después de la interacción del $\mathrm{N}_{2} \mathrm{O}$ en el platino y 
finalmente se calculan los $\Delta q$ de estos átomos. Los valores de las cargas que se reportan para cada interacción solo son para un valor de multiplicidad de espín.

Tabla 5.2.3.1 Análisis de cargas Hirshfeld de las interacciones del $\mathrm{N}_{2} \mathrm{O}$ hacia el isómero I con acercamientos por átomos. Cargas iníciales de los átomos $\left(q_{i}\right)$, cargas finales $\left(q_{f}\right) y$ cambio total de carga $\left(\Delta q=q_{f}-q_{i}\right)$.

\begin{tabular}{|c|c|c|c|c|c|}
\hline \multirow[t]{2}{*}{ Átomos } & \multirow[b]{2}{*}{$\mathbf{q}_{\mathbf{i}}$} & \multicolumn{2}{|c|}{ I-A8N } & \multicolumn{2}{|c|}{${ }^{5}$ I-A8O } \\
\hline & & $\mathbf{q}_{\mathbf{f}}$ & $\Delta \mathbf{q}$ & $\mathbf{q}_{\mathbf{f}}$ & $\Delta \mathbf{q}$ \\
\hline $1 \mathrm{Pt}$ & 0.011 & 0.016 & 0.005 & 0.015 & 0.004 \\
\hline $2 \mathrm{Pt}$ & 0.016 & 0.000 & -0.016 & 0.001 & -0.015 \\
\hline $3 \mathrm{Pt}$ & 0.009 & 0.008 & -0.001 & 0.085 & 0.076 \\
\hline $4 \mathrm{Pt}$ & 0.078 & 0.030 & -0.048 & 0.031 & -0.047 \\
\hline $5 \mathrm{Pt}$ & 0.009 & 0.084 & 0.075 & 0.008 & -0.002 \\
\hline $6 \mathrm{Pt}$ & -0.034 & 0.000 & 0.034 & -0.012 & 0.022 \\
\hline $7 \mathrm{Pt}$ & -0.034 & -0.011 & 0.023 & 0.000 & 0.034 \\
\hline $8 \mathrm{Pt}$ & -0.055 & 0.150 & $\underline{0.205}$ & 0.150 & $\underline{0.205}$ \\
\hline $9 \mathrm{~N}$ & -0.084 & -0.011 & 0.074 & 0.009 & 0.093 \\
\hline $10 \mathrm{~N}$ & 0.196 & 0.009 & -0.187 & -0.010 & -0.206 \\
\hline $11 \mathrm{O}$ & -0.112 & -0.276 & -0.164 & -0.276 & -0.164 \\
\hline
\end{tabular}

(-) Átomos con cambios significativos $\Delta q>0$, carga positiva.

(-) Átomos con cambios significativos $\Delta q<0$, carga negativa.

Como se observa en la tabla anterior, los cambios importantes de carga se localizan en los átomos involucrados en la interacción inicial. Ej. Para la interacción ${ }^{3} I-$ $\underline{A 8 N}$, el átomo que aporta y posee la carga más grande $(\mathrm{q}<0)$ es el átomo $8 \mathrm{Pt}$ suficiente para romper el enlace del $\mathrm{N}_{2} \mathrm{O}$, termina con $\Delta q>0$ (color azul), queda deficiente de carga al finalizar la reacción. Contrario a este átomo los átomos $10 \mathrm{~N}$ y $11 \mathrm{O}$ del $\mathrm{N}_{2} \mathrm{O}$ finalizan con la carga que donó el metal, $\Delta \mathrm{q}<0$ (color rojo). Lo mismo ocurre para ${ }^{5} \underline{I}$ $\underline{A 8 O}$. El resto de los átomos tienen un $\Delta \mathbf{q} \sim 0$ prácticamente sin cambios. Ver Tabla 5.2.3.1 para mayor detalle.

Los principales factores que favorecen para llevar a cabo la reducción del $\mathrm{N}_{2} \mathrm{O}$ es el tipo de acercamiento, la naturaleza del sitio reactivo y el estado electrónico del sistema. Generalmente se favorecieron para multiplicidades de espín 1,3 y 5 . 
Este análisis ayudó explicar y entender el comportamiento de ambas especies, describir cómo se está llevando a cabo la transferencia de carga de la superficie del metal hacia la molécula del $\mathrm{N}_{2} \mathrm{O}$. El principal donador de carga es el metal con $\Delta q>0$ y lo hace a través del átomo más cargado $\left(\mathrm{q}<0\right.$ ), es decir el más reactivo al $\mathrm{N}_{2} \mathrm{O}$ (este átomo posee suficientes e- para donarlos a la molécula del óxido). Contrario al resto de los átomos del metal que se mantiene prácticamente sin cambios, $\Delta q \sim 0$, después de la reacción.

Posteriormente se realizó el análisis de cargas para interacción por enlaces, los casos favorables a la disociación de la molécula del $\mathrm{N}_{2} \mathrm{O}$ se ilustran a continuación en la Figura 5.2.3.2

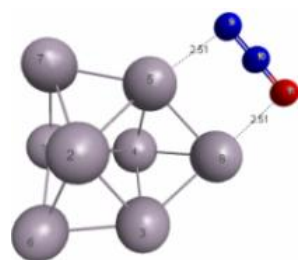

a) ${ }^{5}$ I-E58

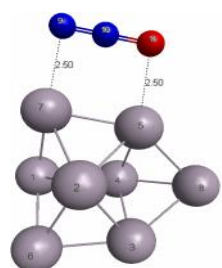

b) ${ }^{7}$ I-E57

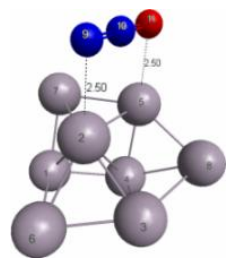

c) ${ }^{3}$ I-E25

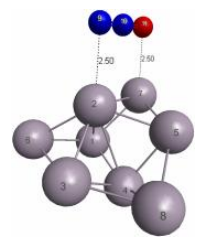

d) ${ }^{3}$ I-E27

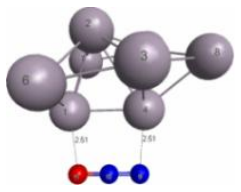

e) ${ }^{3}$ I-E14

Figura 5.2.3.2 Acercamientos favorables a la disociación de la molécula de oxido. Los sitos reactivos son los siguientes, a) I-E58, b) I-E57, c) I-E25, d) I-E27, e) I-E14. Todos los acercamientos a enlaces son paralelos con el eje de la molécula.

En la Tabla 5.2.3.2 se muestran los valores de las Cargas Hirshfeld para las interacciones ilustradas en la Figura 5.3.3.2, cargas iníciales $\left(\mathrm{q}_{\mathrm{i}}\right)$ antes de la interacción, cargas finales $\left(\mathrm{q}_{\mathrm{f}}\right)$ después de la interacción del $\mathrm{N}_{2} \mathrm{O}$ con el isómero I y finalmente se calculan los $\Delta \mathrm{q}$ de todos los átomos. Los valores de las cargas que se reportan para cada interacción solo son para un valor de multiplicidad de espín. 
Tabla 5.2.3.2 Análisis de cargas Hirshfeld de las interacciones del $\mathrm{N}_{2} \mathrm{O}$ hacia el isómero I con acercamientos por enlaces. Cargas iníciales $\left(q_{i}\right)$, cargas finales $\left(q_{f}\right)$ después de la interacción y cambio total de carga $\left(\Delta q=q_{f}-q_{i}\right)$ para todos los átomos.

\begin{tabular}{|c|c|c|c|c|c|c|c|c|c|c|c|}
\hline \multirow[t]{2}{*}{$\overline{\text { Átomo }}$} & \multirow[b]{2}{*}{$q_{i}$} & \multicolumn{2}{|c|}{${ }^{5}$ I-E58 } & \multicolumn{2}{|c|}{${ }^{7}$ I-E57 } & \multicolumn{2}{|c|}{${ }^{3}$ I-E25 } & \multicolumn{2}{|c|}{${ }^{3}$ I-E27 } & \multicolumn{2}{|c|}{${ }^{3}$ I-E14 } \\
\hline & & $q_{f}$ & $\Delta q$ & $\boldsymbol{q}_{f}$ & $\Delta q$ & $q_{f}$ & $\Delta q$ & $q_{f}$ & $\Delta q$ & $q_{f}$ & $\Delta q$ \\
\hline $1 \mathrm{Pt}$ & " 0.011 & $=-0.002$ & 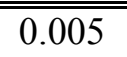 & 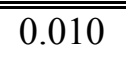 & " & 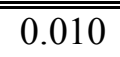 & " -0.001 & "-0.004 & -0.015 & "0.195 & 0.184 \\
\hline $2 \mathrm{Pt}$ & 0.016 & 0.021 & -0.015 & 0.053 & 0.037 & 0.064 & 0.048 & 0.048 & 0.032 & 0.010 & -0.006 \\
\hline $3 \mathrm{Pt}$ & 0.009 & 0.019 & -0.001 & 0.010 & 0.001 & -0.011 & -0.020 & 0.010 & 0.001 & 0.001 & -0.008 \\
\hline $4 \mathrm{Pt}$ & 0.078 & 0.047 & -0.048 & -0.001 & -0.079 & 0.028 & -0.050 & 0.051 & -0.027 & 0.118 & 0.040 \\
\hline $5 \mathrm{Pt}$ & 0.009 & 0.064 & 0.075 & 0.196 & $\underline{0.187}$ & 0.206 & $\underline{0.197}$ & 0.039 & 0.030 & 0.005 & -0.004 \\
\hline $6 \mathrm{Pt}$ & -0.034 & -0.012 & 0.034 & -0.024 & 0.010 & -0.008 & 0.026 & 0.000 & 0.034 & -0.024 & 0.010 \\
\hline $7 \mathrm{Pt}$ & -0.034 & -0.031 & 0.023 & 0.022 & 0.056 & -0.011 & 0.023 & 0.157 & $\underline{0.191}$ & -0.024 & 0.010 \\
\hline $8 \mathrm{Pt}$ & -0.055 & 0.157 & $\underline{0.205}$ & 0.001 & 0.056 & -0.005 & 0.050 & 0.000 & 0.055 & -0.038 & 0.017 \\
\hline $9 \mathrm{~N}$ & -0.084 & 0.009 & 0.074 & 0.004 & 0.088 & 0.004 & 0.088 & -0.005 & 0.079 & 0.016 & $\underline{0.100}$ \\
\hline $10 \mathrm{~N}$ & 0.196 & -0.016 & $\underline{-0.187}$ & -0.021 & $\underline{-0.217}$ & -0.024 & $\underline{-0.220}$ & -0.039 & $\underline{-0.235}$ & -0.003 & -0.199 \\
\hline $11 \mathrm{O}$ & -0.112 & -0.256 & $\underline{-0.164}$ & -0.250 & $\underline{-0.138}$ & -0.253 & $\underline{-0.141}$ & -0.257 & $\underline{-0.145}$ & -0.257 & -0.145 \\
\hline
\end{tabular}

(-) Átomos con cambios significativos $\Delta q>0$, carga positiva.

(-) Átomos con cambios significativos $\Delta q<0$, carga negativa.

Como se puede ver en la Tabla 5.2.3.2, los átomos marcados en azul y rojo son aquellos que muestran cambios importantes en la reacción. Átomos en azul tienen un $\boldsymbol{\Delta} \boldsymbol{q}>\mathbf{0}$, principales donadores de carga. Átomos en rojo tienen un $\boldsymbol{\Delta q}<\mathbf{0}$, átomos que aceptan la carga. A diferencia del análisis anterior en este sitio reactivo se involucran dos átomos, en algunos casos el que posee carga inicial negativa es el principal donador de carga y en otros casos los átomos con carga positiva son quienes aportan la carga, estos cambios se aprecian con más detalle en la tabla anterior.

De igual manera el metal es el principal donador de carga. En este tipo de acercamientos a enlaces se obtuvo la mayor cantidad de sitios reactivos, como se describe en secciones anteriores, el factor principal son: es el tipo de acercamiento, la naturaleza del sitio reactivo y el estado electrónico del sistema los que favorecen la reacción.

Acercamientos por caras no resultaron favorables, su análisis de carga demuestra un $\Delta \boldsymbol{q} \sim \mathbf{0}$ prácticamente sin cambios en todos los átomos de pues de la interacción, por lo 
que no hay transferencia de carga del metal hacia la molécula, haciendo que no se favorezca su disociación.

\subsection{Reacción de disociación del $\mathrm{N}_{2} \mathrm{O}$ catalizada por nanopartículas oxidadas de platino, $\mathrm{Pt}_{8} \mathrm{O}$.}

\subsubsection{Optimización geométrica de la nanopartícula $\mathrm{Pt}_{8} \underline{\mathrm{O}}$.}

Para mejorar la reactividad de la nanopartícula de Pt se propuso oxidarla para incrementar su potencial frente a la reducción del $\mathrm{N}_{2} \mathrm{O}$, en esta sección se estudio el efecto de adsorber un átomo de $\mathrm{O}$ a la estructura del isómero I para determinar su reactividad, por lo que el primer paso fue la oxidación de este, para ello se estudiaron varios sitios de oxidación con átomo de $\mathrm{O}$ sobre el isómero-I, para determinar su geometría más estable.

En una primera parte se absorbe el átomo de $\mathrm{O}$ en los átomos de Pt directamente, en la 2 da el átomo de $\mathrm{O}$ se adsorbe entre dos átomos del isómero formando un enlace tipo puente entre el átomo de oxígeno y los átomos de $\mathrm{Pt}$ y finalmente se adsorbe el átomo de $\mathrm{O}$ entre la superficie de una cara formada por tres átomos de Pt. Estas geometrías del isómero I oxidado fueron optimizadas para las multiplicidades de espín más bajas 1, 3, 5 у 7.

En la Figura 5.3.1.1 se presentan las estructuras optimizadas del isómero I oxidado, en sitios estratégicos, con multiplicidad de espín más estable.

Los diferentes sitios de oxidación se etiquetaron de la siguiente manera: Ej. Para la oxidación en el sitio $\underline{\mathrm{IO}-\mathrm{A} 8}$, 1er termino "I" por isómero I, 2do termino "O" por oxidación, 3er termino "A" por oxidar en átomos del isómero I y finalmente "8" sitio de oxidación en el isómero de Pt. 


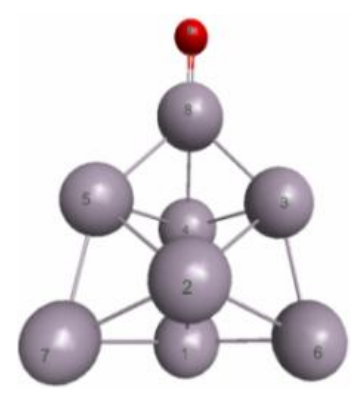

IO-A8

M=5

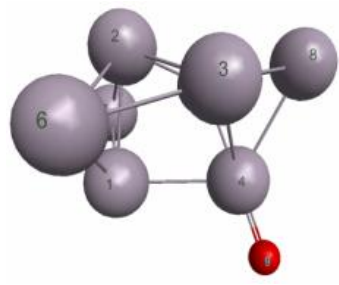

IO-A4

$\mathrm{M}=5$

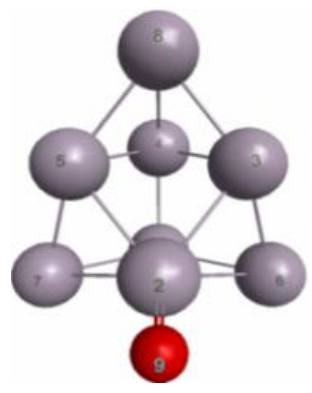

IO-A2

$\mathrm{M}=5$

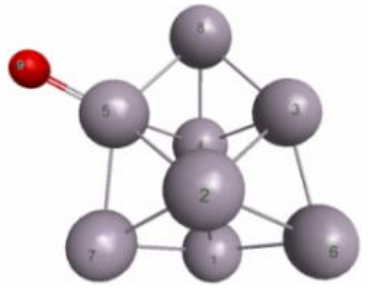

IO-A5

IO-A $3 * *$

$\mathbf{M}=5$

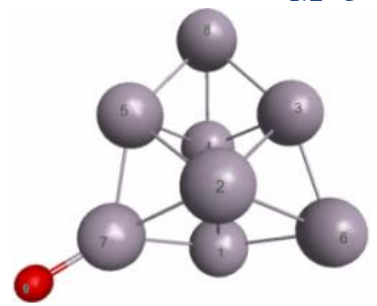

IO-A7

IO-A6**

$\mathbf{M}=\mathbf{5}$

Figura 5.3.1.1 Geometrías estables para la oxidación del isómero I. Con multiplicidades de espín, $M=2 s+1$. (**Adsorciones del átomo de $O$ en posiciones equivalentes al que se adsorbe, por simetría del isómero I sin oxidar se generan estos sitios equivalentes).

En esta primera parte se oxida en un átomo del metal directamente. Figura 5.3.1.1. En esta oxidación se obtiene el isómero de más baja energía para IO-A7 con multiplicidad de espín 5, con energías muy similares de 0.93 y $1.19 \mathrm{kcal} / \mathrm{mol}$ con multiplicidad de espín 1 y 3, lo mismo ocurre en IO-A8 para M 5 la energía es $<2$ $\mathrm{kcal} / \mathrm{mol}$. Al igual que para M 7 en IO-A7 y IO-A8 con M 3. Otros sitios como IO-A7 y IO-A8 con multiplicidad de espín 7 y 3 respectivamente presentan energías $\sim 3 \mathrm{kcal} / \mathrm{mol}$ (cercanas a la degeneración)

En la Tabla 5.3.1.2 se muestran los parámetros de energía del isómero oxidado. Sin incluir los que tiene equivalencia, los resultados son los mismos en cada caso. 
Tabla 5.3.1.1 Parámetros energéticos de estructuras optimizadas del isómero oxidado, $P t_{8} O$. Energías de adsorción $\left(E_{\text {ads }}\right)$ y energía relativa $\left(E_{\text {relativa }}\right)$.

\begin{tabular}{cccc}
\hline \hline $\begin{array}{c}\text { Tipo de } \\
\text { interacción }\end{array}$ & $\begin{array}{c}\mathbf{M} \\
\mathbf{2 s + 1}\end{array}$ & $\begin{array}{c}\mathbf{E}_{\mathbf{a d s}} \\
\mathbf{k c a l} / \mathbf{m o l}\end{array}$ & $\begin{array}{c}\mathbf{E}_{\text {relativa }} \\
\mathbf{k c a l} / \mathbf{m o l}\end{array}$ \\
\hline \hline & 1 & -91.07 & 4.92 \\
IO-A8 & 3 & -92.94 & 3.05 \\
& 5 & -94.13 & 1.86 \\
& 7 & -90.45 & 5.54 \\
\hline \multirow{2}{*}{ IO-A4 } & 1 & -84.38 & 11.61 \\
& 3 & -88.45 & 7.54 \\
& 5 & -89.03 & 6.96 \\
IO-A2 & 7 & --- & $*$ \\
& 1 & -80.52 & 15.47 \\
& 3 & -82.42 & 13.57 \\
& 5 & -83.28 & 12.71 \\
IO-A5 & 7 & -82.56 & 13.43 \\
\hline IO-A7 & 1 & -85.19 & 10.80 \\
& 3 & -89.10 & 6.89 \\
& 5 & -89.09 & 6.90 \\
& 7 & -86.48 & 9.51 \\
\hline & 1 & -95.06 & 0.93 \\
& 3 & -94.80 & 1.19 \\
& 5 & -95.99 & 0.00 \\
& 7 & -92.68 & 3.31 \\
\hline
\end{tabular}

* Problemas de convergencia no hay resultados.

Los valores de frecuencia de vibración que se reportan son reales y máximos en todos los casos, para la interacción de más baja energía (IO-A7, M=5) su valor es 842 $\mathrm{cm}^{-1}$, para el resto de las interacciones este valor decrece, corresponde a un modo normal de vibración del átomo de $\mathrm{O}$ enlazado a un átomo del metal, estiramiento del enlace Pt-O.

Las distancias Pt-O se mantiene prácticamente invariables entre los 1.76 a $1.80 \AA$, y las distancias promedio Pt-Pt se encuentran entre los 2.58 y $2.64 \AA$.

En la Tabla 5.3.1.2 se muestran los parámetros geométricos de las adsorciones del átomo de $\mathrm{O}$ en la superficie del isómero oxidado. 
Tabla 5.3.1.2 Parámetros geométricos para estructuras optimizadas del isómero oxidado, $\mathrm{Pt}_{8} \mathrm{O}$. Multiplicidad (M), Frecuencias de vibración (v) y distancias de enlace (d).

\begin{tabular}{|c|c|c|c|c|c|}
\hline $\begin{array}{c}\text { Tipo de } \\
\text { interacción }\end{array}$ & $\begin{array}{c}M \\
2 s+1\end{array}$ & $\begin{array}{l}v^{P t-O} \\
\mathrm{~cm}^{-1}\end{array}$ & $\begin{array}{c}\mathbf{d}_{\text {Pt-O }} \\
\AA\end{array}$ & $\begin{array}{c}\mathbf{d P t}_{\mathbf{P t}} \\
\AA\end{array}$ & $\underset{\AA}{ \pm \underset{\mathbf{A}}{\mathbf{\AA}}}$ \\
\hline \multirow{4}{*}{ IO-A8 } & 1 & 855 & 1.75 & 2.59 & 0.06 \\
\hline & 3 & 850 & 1.75 & 2.62 & 0.11 \\
\hline & 5 & 842 & 1.75 & 2.58 & 0.04 \\
\hline & 7 & 834 & 1.76 & 2.59 & 0.04 \\
\hline \multirow{4}{*}{ IO-A4 } & 1 & 812 & 1.77 & 2.58 & 0.06 \\
\hline & 3 & 810 & 1.77 & 2.58 & 0.04 \\
\hline & 5 & 814 & 1.77 & 2.59 & 0.04 \\
\hline & 7 & --- & --- & --- & $*$ \\
\hline \multirow{4}{*}{ IO-A2 } & 1 & 823 & 1.76 & 2.62 & 0.12 \\
\hline & 3 & 778 & 1.79 & 2.61 & 0.11 \\
\hline & 5 & 769 & 1.80 & 2.59 & 0.06 \\
\hline & 7 & 776 & 1.79 & 2.60 & 0.07 \\
\hline \multirow{4}{*}{ IO-A5 } & 1 & 818 & 1.77 & 2.62 & 0.13 \\
\hline & 3 & 794 & 1.78 & 2.62 & 0.14 \\
\hline & 5 & 796 & 1.78 & 2.61 & 0.07 \\
\hline & 7 & 793 & 1.78 & 2.64 & 0.13 \\
\hline \multirow{4}{*}{ IO-A7 } & 1 & 846 & 1.76 & 2.59 & 0.09 \\
\hline & 3 & 829 & 1.76 & 2.59 & 0.08 \\
\hline & 5 & 842 & 1.76 & 2.58 & 0.09 \\
\hline & 7 & 842 & 1.76 & 2.61 & 0.06 \\
\hline
\end{tabular}

* Problemas de convergencia no hay resultados.

Posteriormente se realizó la adsorción del átomo de oxígeno ahora entre dos átomos de Pt como se muestra en la figura 5.3.1.2, tales adsorciones se etiquetan de la siguiente manera: Ej. Para la adsorción IO-E48, 1er termino "I" por isómero I, 2do termino "O" por oxidación, 3er termino "E" por adsorción entre dos átomos y finalmente "48" sitio de adsorción del átomo de $\mathrm{O}$ entre los átomos de Pt. 


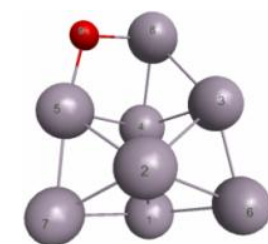

IO-E58

IO-E38**

$\mathbf{M}=3$

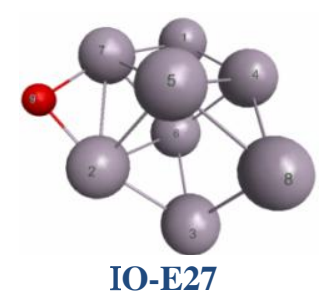

IO-E27

IO-E26**

$\mathbf{M}=7$

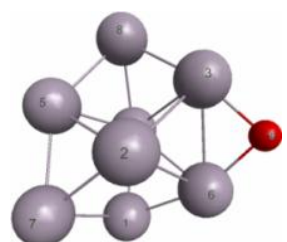

IO-E36

IO-E57**

$\mathbf{M}=\mathbf{3}$

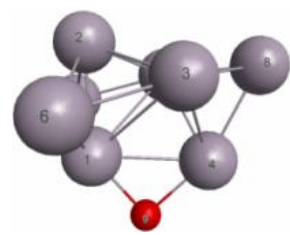

IO-E14

$\mathrm{M}=3$

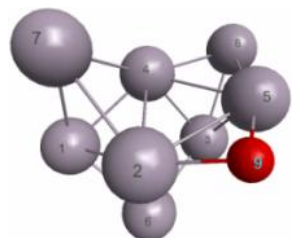

IO-E25

IO-E23**

$\mathbf{M}=1$

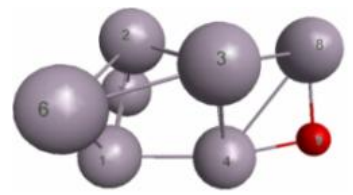

IO-E48

$\mathrm{M}=3$

Figura 5.3.1.2 Geometrías más estables para la adsorción del átomo de oxígeno sobre el isómero I, en diferentes sitios formados por enlaces Pt-Pt, con sus multiplicidades de espín, $M=2 s+1$. (**Adsorciones del átomo de $O$ en posiciones equivalentes al que se adsorbe, por simetría del isómero Isin oxidar se generan estos sitios equivalentes).

La estructura del isómero de platino oxidado $\left(\mathrm{Pt}_{8} \mathrm{O}\right)$ más estable se obtiene para el isómero, IO-E25, con multiplicidad de espín 1, Figura 5.3.1.2. Para M 3 se obtiene una energía muy similar, $<$ de $3 \mathrm{kcal} / \mathrm{mol}$. Otro isómero con energía muy cercana al más estable(menos de $3 \mathrm{kcal} / \mathrm{mol}$ ), se obtiene para IO-E36 con M 1,y con $\sim 3 \mathrm{kcal} / \mathrm{mol}$ para M 5 degeneración con el más estable.

La energía de adsorción $\left(\mathrm{E}_{\mathrm{ads}}\right)$ de la estructura de más baja energía es $-98.97 \mathrm{kcal} / \mathrm{mol}$, con $<1 \mathrm{kcal} / \mathrm{mol}$ y multiplicidad de espín 3 el mismo isómero, IO-E25. Otro isómero IO-E36 presentan energías muy cercanas con tan solo 1.33 y $3.42 \mathrm{kcal} / \mathrm{mol}$ por arriba con M 3 y 5 respectivamente. El resto de las adsorciones son menos estables que las mencionadas ya que presentan energías ( $>5 \mathrm{kcal} / \mathrm{mol}$ ) mayores que la del fundamental.

En la Tabla 5.3.1.3 se resumen los parámetros de energía determinados para estos tipos de adsorciones, excepto para los de sitios equivalentes. 
Tabla 5.3.1.3 Parámetros energéticos de estructuras optimizadas de $\mathrm{Pt}_{8} \mathrm{O}$ con adsorción del átomo de $O$ en enlaces Pt-Pt del isómero I. Energías de adsorción $\left(E_{a d s}\right)$ y energía relativa $\left(\boldsymbol{E}_{\text {relativa }}\right)$.

\begin{tabular}{|c|c|c|c|}
\hline $\begin{array}{c}\text { Tipo de } \\
\text { interacción }\end{array}$ & $\begin{array}{c}M \\
2 s+1\end{array}$ & $\begin{array}{c}\mathbf{E}_{\mathrm{ads}} \\
\mathrm{kcal} / \mathrm{mol}\end{array}$ & $\begin{array}{c}\text { E}_{\text {relativa }} \\
\mathrm{kcal} / \mathrm{mol}\end{array}$ \\
\hline \multirow{4}{*}{ IO-E48 } & 1 & -81.99 & 16.97 \\
\hline & 3 & -86.51 & 12.46 \\
\hline & 5 & -85.83 & 13.14 \\
\hline & 7 & -82.45 & 16.52 \\
\hline \multirow{4}{*}{ IO-E58 } & 1 & -88.45 & 10.51 \\
\hline & 3 & -89.67 & 9.29 \\
\hline & 5 & -88.62 & 10.35 \\
\hline & 7 & -84.07 & 14.90 \\
\hline \multirow{4}{*}{ IO-E25 } & 1 & -98.97 & 0.00 \\
\hline & 3 & -98.22 & 0.74 \\
\hline & 5 & -83.55 & 15.41 \\
\hline & 7 & -93.91 & 5.05 \\
\hline \multirow{4}{*}{ IO-E14 } & 1 & -92.94 & 6.03 \\
\hline & 3 & -98.22 & 0.75 \\
\hline & 5 & --- & $*$ \\
\hline & 7 & -93.94 & 5.03 \\
\hline \multirow{4}{*}{ IO-E27 } & 1 & -81.34 & 17.63 \\
\hline & 3 & -88.00 & 10.97 \\
\hline & 5 & -83.08 & 15.89 \\
\hline & 7 & -92.97 & 5.99 \\
\hline \multirow{4}{*}{ IO-E36 } & 1 & --- & $*$ \\
\hline & 3 & -97.64 & 1.33 \\
\hline & 5 & -95.54 & 3.42 \\
\hline & 7 & -87.94 & 11.03 \\
\hline
\end{tabular}

* Problemas de convergencia no se muestran resultados.

Las frecuencias de vibración reportadas son todas reales y máximas. Sus valores $\sim 550 \mathrm{~cm}^{-1}$, menor que en el caso anterior, ahora el átomo de oxígeno esta enlazado a dos átomos de Pt, tiene menos libertad de movimiento. Los valores de estas frecuencias son debidas a un modo normal de vibración, principalmente al estiramiento de los enlaces Pt-O. Las distancias promedio đ đet-Pt del estado más baja energía (IO-E25 y $\mathrm{M}=1$ ) son $2.62 \pm 0.09 \AA$, y la distancia del enlace O-Pt ahora $>1.91 \AA$, se forma un 
enlace más débil con los átomos de $\mathrm{Pt}$, en el resto de las adsorciones, las distancias promedio Pt-Pt oscilaron entre $\sim 2.60 \AA$.

En la Tabla 5.3.1.4 se resumen los parámetros geométricos de la adsorción del átomo de $\mathrm{O}$ en la superficie del isómero I, formando una adsorción tipo puente entre el átomo de $\mathrm{O}$ y dos átomos de Pt.

Tabla 5.3.1.4 Parámetros geométricos de estructuras optimizadas de $\mathrm{Pt}_{8} \mathrm{O}$ con adsorción del átomo de $O$ en enlaces Pt-Pt del isómero I. Multiplicidad (M), frecuencias de vibración y distancias de enlace (d).

\begin{tabular}{|c|c|c|c|c|c|}
\hline $\begin{array}{c}\text { Tipo de } \\
\text { interacción }\end{array}$ & $\begin{array}{c}M \\
2 s+1\end{array}$ & $\begin{array}{l}v^{P t-O} \\
\mathrm{~cm}^{-1}\end{array}$ & $\begin{array}{c}\mathbf{d}_{\mathrm{Pt}-\mathrm{O}} \\
\AA\end{array}$ & $\begin{array}{c}\mathbf{d}_{\mathrm{Pt}-\mathrm{Pt}} \\
\AA\end{array}$ & $\begin{array}{c} \pm \mathbf{d}_{\mathbf{P t}-\mathrm{Pt}} \\
\stackrel{\AA}{\mathrm{A}}\end{array}$ \\
\hline \multirow{4}{*}{ IO-E48 } & 1 & 599 & 1.93 & 2.62 & 0.1 \\
\hline & 3 & 587 & 1.93 & 2.61 & 0.09 \\
\hline & 5 & 583 & 1.95 & 2.61 & 0.09 \\
\hline & 7 & 579 & 1.94 & 2.65 & 0.14 \\
\hline \multirow{4}{*}{ IO-E58 } & 1 & 552 & 1.93 & 2.61 & 0.12 \\
\hline & 3 & 554 & 1.93 & 2.61 & 0.10 \\
\hline & 5 & 599 & 1.95 & 2.62 & 0.08 \\
\hline & 7 & 558 & 1.91 & 2.67 & 0.14 \\
\hline \multirow{4}{*}{ IO-E25 } & 1 & 588 & 1.91 & 2.62 & 0.09 \\
\hline & 3 & 581 & 1.94 & 2.67 & 0.14 \\
\hline & 5 & 564 & 1.99 & 2.60 & 0.09 \\
\hline & 7 & 567 & 1.95 & 2.77 & 0.14 \\
\hline \multirow{4}{*}{ IO-E14 } & 1 & 599 & 1.92 & 2.66 & 0.12 \\
\hline & 3 & 581 & 1.94 & 2.67 & 0.14 \\
\hline & 5 & --- & --- & --- & * \\
\hline & 7 & 567 & 1.95 & 2.67 & 0.14 \\
\hline \multirow{4}{*}{ IO-E27 } & 1 & 585 & 1.93 & 2.61 & 0.09 \\
\hline & 3 & 585 & 1.93 & 2.61 & 0.09 \\
\hline & 5 & 574 & 1.93 & 2.62 & 0.10 \\
\hline & 7 & 569 & 1.96 & 2.60 & 0.08 \\
\hline \multirow{4}{*}{ IO-E36 } & 1 & --- & --- & --- & * \\
\hline & 3 & 572 & 1.96 & 2.62 & 0.10 \\
\hline & 5 & 569 & 1.95 & 2.63 & 0.13 \\
\hline & 7 & 567 & 1.96 & 2.64 & 0.13 \\
\hline
\end{tabular}

* Problemas de convergencia no hay resultados. 
Este resultado es favorable en estos sitios por que los átomos que forman el sitio tiene cargas positivas y pequeñas que contrarresta la carga negativa del átomo de $\mathrm{O}$, los efectos de repulsión son mínimos dando estabilidad a la molécula, en el resto de los sitios los átomos tienen cargas opuestas o negativas ambas, lo que genera inestabilidad y valores de energías más grandes.

Finalmente se presentan los resultados de adsorción del átomo de en la superficie de una cara de la nanopartícula, como se muestra en la figura 5.3.1.3

Las diferentes adsorciones se etiquetaron de la siguiente manera: Para la adsorción IO$\underline{\mathrm{C} 236}$, 1er termino "I" por isómero I, 2do termino "O" por oxidación, 3er termino "C" por adsorción en una cara y finalmente "236" sitio de adsorción del átomo de $\mathrm{O}$ entre los átomos que forman la cara.

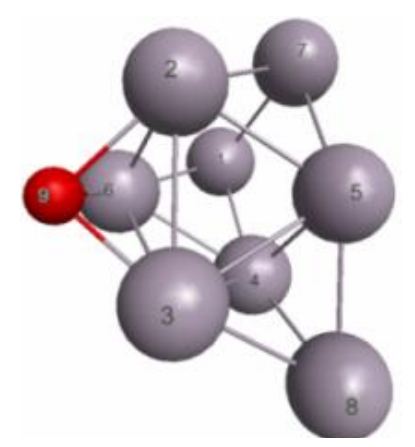

IO-C236

$\mathrm{IO}-\mathrm{C} 257^{* *}$

$\mathbf{M}=\mathbf{3}$

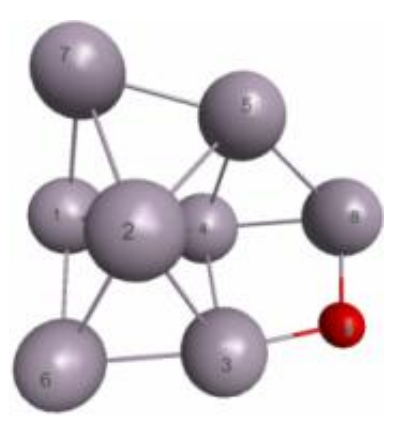

IO-C348

$\mathrm{IO}-\mathrm{C} 458 * *$

$\mathbf{M}=\mathbf{3}$

Figura 5.3.1.3 Geometrías más estables para la adsorción de un átomo de oxígeno sobre algunas caras del isómero I de Pt, con sus multiplicidades de espín, $M=2 s+1$. (**Adsorciones del átomo de $O$ en posiciones equivalentes al que se adsorbe, por la simetría del isómero I sin oxidar se generan estos sitios equivalentes).

Solo se estudian dos casos, el de más baja energía es IO-C3480 con multiplicidad de espín 3, el resto de los valores están por arriba de las $4.84 \mathrm{kcal} / \mathrm{mol}$. En la Tabla 5.3.1.5 se resumen los resultados de energía de los dos tipos de adsorción estudiados, de estos dos, el de la adsorción IO-C348 con multiplicidad 3 presenta más baja energía. 
Tabla 5.3.1.5 Parámetros energéticos de estructuras de $\mathrm{Pt}_{8}$ con adsorción del átomo de $\mathrm{O}$ en caras del isómero I. Energías de adsorción $\left(E_{\text {ads }}\right)$ y energía relativa $\left(E_{\text {relativa }}\right)$.

\begin{tabular}{cccc}
\hline \hline $\begin{array}{c}\text { Tipo de } \\
\text { interacción }\end{array}$ & $\begin{array}{c}\mathbf{M} \\
\mathbf{2 s + 1}\end{array}$ & $\begin{array}{c}\mathbf{E}_{\text {ads }} \\
\mathbf{k c a l} / \mathbf{m o l}\end{array}$ & $\begin{array}{c}\mathbf{E}_{\text {relativa }} \\
\mathbf{k c a l} / \mathbf{m o l}\end{array}$ \\
\hline \hline & 1 & -84.24 & 5.40 \\
IO-C236 & 3 & -84.80 & 4.84 \\
& 5 & -83.55 & 6.09 \\
\hline & 7 & --- & --- \\
\hline \multirow{2}{*}{ IO-C348 } & 1 & -81.99 & 7.65 \\
& 3 & -89.64 & 0.00 \\
& 5 & --- & $*$ \\
\hline \hline
\end{tabular}

Los parámetros geométricos de esta adsorción se muestran en la Tabla 5.3.1.7 Las frecuencias de vibración que se reportan son reales como en todos los casos y las de mayor intensidad. Sus valores aún mucho menores que en los dos casos anteriores, debido a que ahora el átomo de oxígeno comparte tres enlaces con el metal, tiene menos libertad de movimiento. Al igual que la distancia Pt-O ahora aumenta y las distancias promedio Pt-Pt $\sim 2.60 \AA$.

Tabla 5.3.1.6 Parámetros geométricos de estructuras de $\mathrm{Pt}_{8} \mathrm{O}$ con adsorción del átomo de $\mathrm{O}$ en las caras del isómero I. Multiplicidad de espín (M), frecuencias de vibración (v) y distancias de enlace $(d)$.

\begin{tabular}{|c|c|c|c|c|c|}
\hline $\begin{array}{c}\text { Tipo de } \\
\text { interacción }\end{array}$ & $\begin{array}{c}\mathrm{M} \\
2 \mathrm{~s}+1\end{array}$ & $\begin{array}{l}v^{P t-O} \\
\mathrm{~cm}^{-1}\end{array}$ & $\begin{array}{c}\mathbf{d P t}_{\mathbf{P t}} \\
\AA\end{array}$ & $\begin{array}{c}\mathbf{d}_{\mathbf{P t}-\mathbf{P t}} \\
\AA\end{array}$ & $\begin{array}{l} \pm \mathbb{đ}_{\mathbf{P t}-\mathrm{Pt}} \\
\stackrel{\AA}{\mathrm{A}}\end{array}$ \\
\hline \multirow{4}{*}{ IO-F236 } & 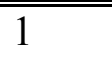 & 4999 & 2.00 & 2.67 & 0.14 \\
\hline & 3 & 508 & 2.08 & 2.63 & 0.10 \\
\hline & 5 & 563 & 1.99 & 2.60 & 0.09 \\
\hline & 7 & --- & --- & --- & $*$ \\
\hline \multirow{4}{*}{ IO-F348 } & 1 & 599 & 1.93 & 2.62 & 0.08 \\
\hline & 3 & 468 & 1.93 & 2.61 & 0.10 \\
\hline & 5 & --- & --- & --- & $*$ \\
\hline & 7 & --- & --- & --- & $*$ \\
\hline
\end{tabular}

* Problemas de convergencia no se muestran resultados. 
De manera general concluimos que de todas las adsorciones probadas, los más estables fueron los isómeros oxidados, IO-E25 (M 1 y 3), IO-E14 (M 3) y IO-E36 (M 3) con energías muy cercanas, con más de un valor de multiplicidad de espín, generando así varias estructuras con la misma energía. Por ahora solo se considerara el primero IOE25 con M 1 (más bajo en energía) para el estudio de las adsorciones del $\mathrm{N}_{2} \mathrm{O}$ por cuestiones de tiempo.

Una de las características que presentaron estos sitios en el isómero I sin oxidar es: la carga negativa en ambos átomos y cargas opuestas pero pequeñas, esto hace que se minimicen los efectos de expulsión entre los átomos del metal y el átomo de oxígeno generando así las estructuras más estable que el resto.

Además de los parámetros reportados en las tablas anteriores, se determina si las estructuras de los isómeros oxidados sufren cambios después de adsorber el átomo de $\mathrm{O}$ en la nanopartícula de $\mathrm{Pt}_{8}$, los resultados son menores del $3 \%$ de deformación, lo que significa que la estructura geométrica de la nanopartícula se conserva. A continuación se realizó el estudio de la adsorción del óxido nitroso sobre el isómero oxidado de platino.

\subsubsection{Estudio de la disociación del $\mathrm{N}_{2} \mathrm{O}$ sobre la nanopartícula de $\mathrm{Pt}_{8} \mathrm{O}$. Identificación de sitios de activación por $\mathrm{N}_{2} \mathrm{O}$.}

El estudio de la adsorción del óxido nitroso sobre el isómero oxidado de $\mathrm{Pt}$, se realizó a partir de la geometría obtenida en la sección anterior de más baja energía, IOE25 M 1, Figura 5.3.2.1, aunque para este isómero oxidado se determinaron otras geometrías con energías muy cercanas $(<3 \mathrm{kcal} / \mathrm{mol})$ como se discutió en la sección anterior, solo se considera el estudio de la adsorción del $\mathrm{N}_{2} \mathrm{O}$ en, IO-E25 y M 1. La geometría de este nuevo isómero oxidado ahora no presenta ningún elemento de simetría por lo que se consideran todos los sitios posibles de interacción. 
En la siguiente figura 5.3.2.1 se muestra la geometría de más baja energía para el isómero oxidado de $\mathrm{Pt}_{8}$ (IO-E25) y su distribución de carga Hirshfeld.

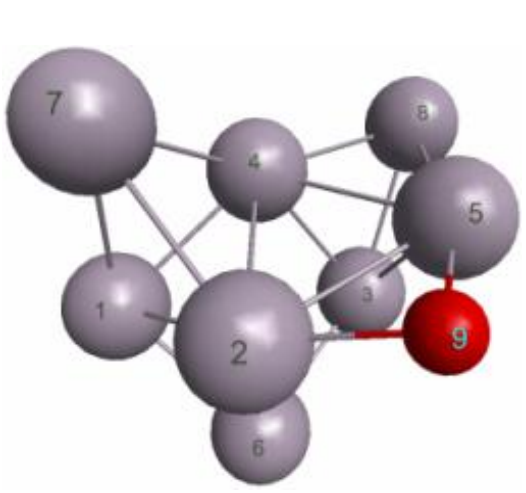

a)

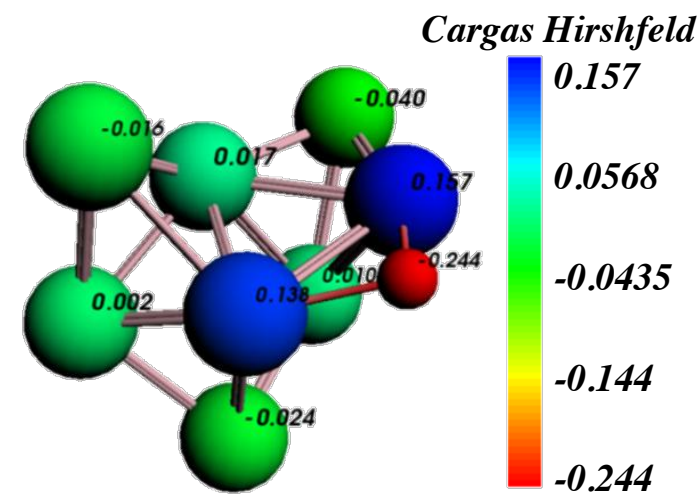

b)

Figura 5.3.2.1 a) Estructura geométrica de mínima energía para el isómero Ioxidado, ${ }^{1}$ IO-E25 $\left({ }^{1}\right.$ IO-E23**), b) Distribución de carga Hirshfeld en el isómero oxidado (zonas rojas ricas en e- y zonas azules deficientes). **Sitio de oxidación equivalente al indicado, por simetría inicial del isómero Isin oxidar.

Este estudio se divide en tres partes, la primera consiste en acercar la molécula del $\mathrm{N}_{2} \mathrm{O}$ hacia el isómero oxidado $\mathrm{Pt}_{8} \mathrm{O}$ (IO-E25), interaccionando un átomo de $\mathrm{Pt}$ con cada uno de los átomos del $\mathrm{N}_{2} \mathrm{O}$. Para determinar sus sitios reactivos frente a la disociación del óxido. A continuación se detallan los tipos de interacción y los resultados obtenidos de la reacción de $\mathrm{N}_{2} \mathrm{O}$ con la nanopartícula de Pt oxidada (Figura 5.3.2.1) en esta primera parte.

\section{Interacción directa con átomos.}

En esta primera parte se hace interaccionar el $\mathrm{N}_{2} \mathrm{O}$ con $\mathrm{Pt}_{8} \mathrm{O}$ a una distancia inicial de $3 \AA$. Acercando cada uno de los átomos del óxido nitroso a cada uno de los posibles sitios reactivos del isómero oxidado (IO-E25). 
En la Figura 5.3.2.2 se muestran los acercamientos más favorables que dieron lugar a la disociación del enlace $\mathrm{N}-\mathrm{O}$ del $\mathrm{N}_{2} \mathrm{O}$, estos resultados se favorecieron preferentemente para multiplicidades de spin 3, 5 y 7 . El resto de las interacciones no fueron favorables, los resultados se resume en el anexo 9.1.6

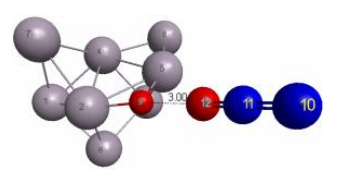

IO-A9O

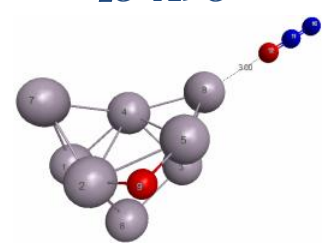

IO-A8O

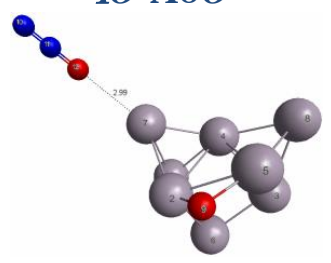

IO-A7O
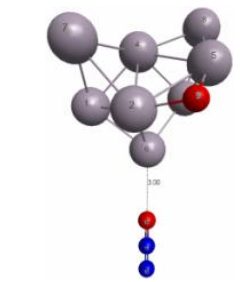

IO-A6O
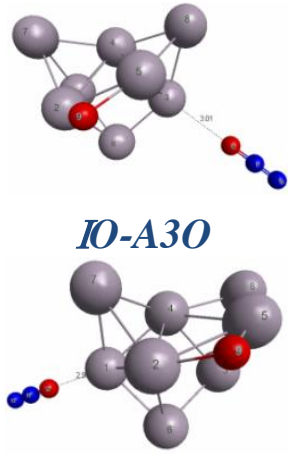

IO-A1O

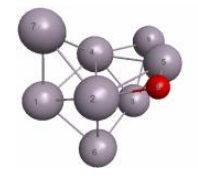

$M=1$

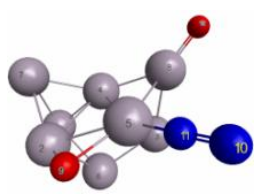

$M=1$

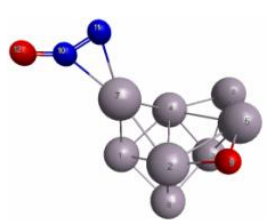

$M=1$

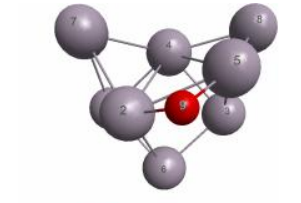

00

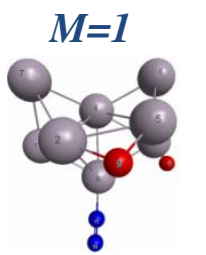

$M=1$

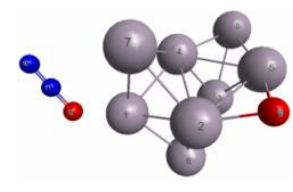

$M=1$

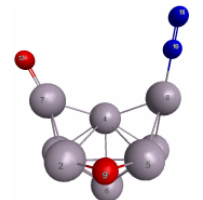

$M=3$
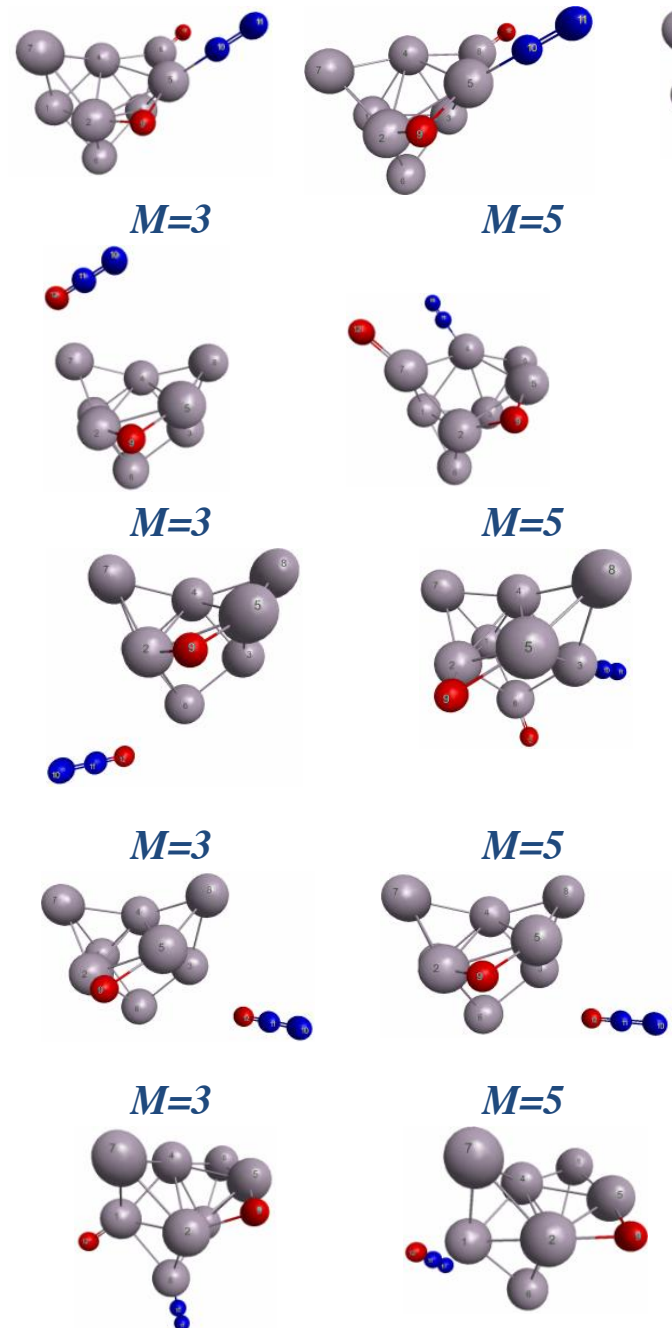

$M=3$

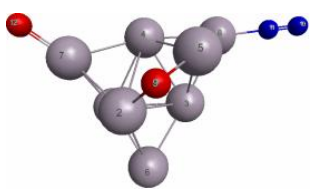

$M=5$

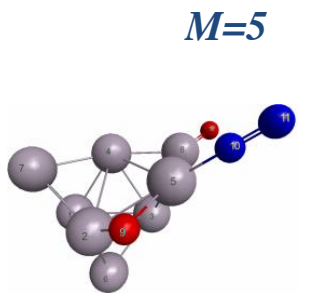

$M=5$
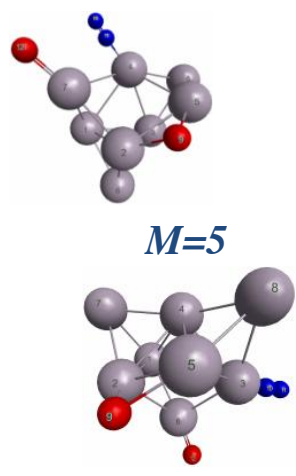
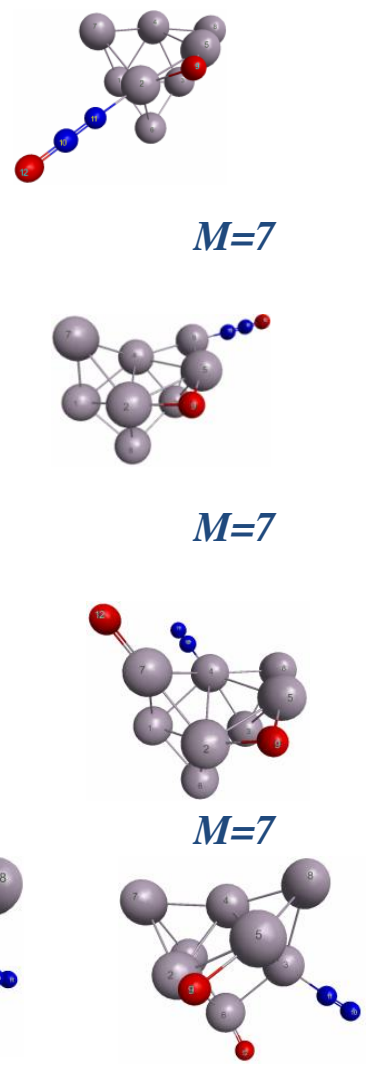

Figura 5.3.2.2 Resultados de interacción de $\mathrm{Pt}_{8} \mathrm{O}+\mathrm{N}_{2} \mathrm{O}$ en diferentes átomos Pt por el lado del $\mathrm{O}$, con distintas multiplicidades de espín $(M=2 s+1)$. (*) Interacciones con problemas de convergencia, no se muestran resultados. 
Los diferentes tipos de interacciones se etiquetaron de la siguiente manera. Ej. Para la interacción IO-A9O, 1er termino "I" por isómero I, 2do termino "O" por oxidación, 3er termino "A" por adsorción en átomos de Pt, 4to "9" sitio de interacción en el isómero oxidado y finalmente "므" átomo del óxido nitroso que interacciona con el isómero oxidado.

Descripción de acercamientos de Figura 5.3.2.2

IO-A90: Directamente hacia el $\mathrm{O}_{\text {ads }}$ (sitio nueve) del isómero oxidado (IO-E25) se aproxima el átomo de $\mathrm{O}$ del $\mathrm{N}_{2} \mathrm{O}$, los resultados fueron, reducción para multiplicidad de espín 3 y 5 con energías de -68.54 y $-58.39 \mathrm{kcal} / \mathrm{mol}$ respectivamente. Para M 1 y 7 se obtiene adsorción sin disociar a la molécula de $\mathrm{N}_{2} \mathrm{O}$. Los productos de reacción $\mathrm{O}$ y $\mathrm{N}_{2}$ se adsorben ala nanopartícula de $\mathrm{Pt}_{8} \mathrm{O}$, en átomos alejados al sitio de interacción inicial.

IO-A80: La molécula del $\mathrm{N}_{2} \mathrm{Ose}$ acerca hacia el átomo o sitio 8 del isómeroIO-E25, genera la reducción del óxido para multiplicidad de espín 1, 3 y 5 con $E_{a d s}$ de $-57.34,-56.33$ y $-55.02 \mathrm{kcal} / \mathrm{mol}$ respectivamente. En M 7 solo se obtiene adsorción sin disociar. Los productos de la reacción se adsorben en los sitios de interacción inicial y en átomos vecinos.

IO-A70: Acercando la molécula de $\mathrm{N}_{2} \mathrm{O}$ por el átomo de oxígeno hacia el átomo ó sitio 7 del isómero IO-E25, se genera la disociación del enlace $\mathrm{N}-\mathrm{O}$ en multiplicidad de espín 5 y 7 las energías obtenidas son de -62.04 y -45.90 kcal/mol. Para multiplicidad de espín 1, solo adsorción sin disociar enlazándose ambos átomos de $\mathrm{N}$ a un átomo de Pt (enlace tipo caballo). En multiplicidad de espín 3 hay adsorción por el átomo de oxígeno, pero la E $>0$. Los productos de la reacción se adsorben en los sitios de interacción inicial y en átomos vecinos. 
IO-A6O: El átomo de $\mathrm{O}$ del óxido $\mathrm{N}_{2} \mathrm{O}$ interacciona con el átomo o sitio 6 del isómeroI O-E25, se obtiene disociación solo en multiplicidad de espín 5 y 7 con energías de -62.07 y $-53.54 \mathrm{kcal} / \mathrm{mol}$ respectivamente. En multiplicidad de espín 1 y 3 solo hay ligera adsorción por el átomo de oxígeno con $\mathrm{E}_{\mathrm{dis}}<0$. Los productos de la reacción, $\mathrm{O}$ y $\mathrm{N}_{2}$ se adsorben en el sitios de interacción inicial y átomos vecinos.

IO-A3O: El átomo de $\mathrm{O}$ del $\mathrm{N}_{2} \mathrm{O}$ se acerca al átomo o sitio 3del isómero IO-E25, la reducción del óxido solo se presenta en multiplicidad de espín 1 con una energía de disociación de $-59.12 \mathrm{kcal} / \mathrm{mol}$. En el resto de las multiplicidades de espín solo con adsorción. Los productos de la reacción: $\mathrm{O}$ y $\mathrm{N}_{2}$ se adsorben en la nanopartícula.

IO-A10: $\mathrm{El}$ átomo de $\mathrm{O}$ del $\mathrm{N}_{2} \mathrm{O}$ se acerca directamente hacia el átomo ó sitio 1 del isómero oxidado, generando reducción del óxido solo para multiplicidad de espín 3 con una energía de $-56.34 \mathrm{kcal} / \mathrm{mol}$. En M 1 solo hay ligera adsorción por $\mathrm{O}$ con energía muy pequeña (fisisorción), en M 5 hay ligera adsorción por $\mathrm{N}$, pero la reacción es endotérmica. Los productos de la reacción se adsorben en los sitios de interacción inicial y en átomos vecinos nuevamente.

En la Tablas 5.3.2.1 se resumen los parámetros de energía determinados para cada una de estas interacciones que resultaron favorables en la disociación del enlace $\mathrm{N}-\mathrm{O}$ en la molécula del óxido nitroso. El resto de las interacciones no favorables se muestran en el anexo 9.1.6

El estado de menor energía se determina para la interacción del átomo de $\mathrm{O}_{\text {ads }}$ ó sitio 9 en el isómero IO-E25, con el átomo de $\mathrm{O}$ del $\mathrm{N}_{2} \mathrm{O}$, A9-O, con multiplicidad de espín triplete (Figura 5.3.2.2), con una $\mathrm{E}_{\mathrm{dis}}$ de $-68.54 \mathrm{kcal} / \mathrm{mol}$. El resto de los acercamientos no presentan degeneración con el estado fundamental. El valor más 
cercano esta $6.47 \mathrm{kcal} / \mathrm{mol}$ por encima de este y se presenta para $\mathrm{IO}-\mathrm{A} 6 \mathrm{O}$ con multiplicidad de espín 5.

Tabla 5.3.2.1 Parámetros energéticos de la interacción del $\mathrm{N}_{2} \mathrm{O}$ sobre átomos de Pt del isómero oxidado, IO-E25, por el átomo de $O$ del óxido. Energías de disociación $\left(E_{d i s}\right)$ y energía relativa $\left(\boldsymbol{E}_{\text {relativa }}\right)$.

\begin{tabular}{|c|c|c|c|c|}
\hline Tipo de interacción & $\begin{array}{c}M \\
2 s+1\end{array}$ & $\begin{array}{c}\mathrm{E}_{\mathrm{dis}} \\
\mathrm{kcal} / \mathrm{mol}\end{array}$ & $\begin{array}{c}\text { E }_{\text {relativa }} \\
\text { kcal/mol }\end{array}$ & $\begin{array}{c}\text { Enlace } \\
\text { /disociación }\end{array}$ \\
\hline \multirow{4}{*}{ IO-A9O } & $\overline{11}$ & -3.35 & 65.19 & si/no \\
\hline & 3 & -68.54 & 0.00 & $\mathrm{si} / \mathrm{si}$ \\
\hline & 5 & -58.39 & 10.15 & $\mathrm{si} / \mathrm{si}$ \\
\hline & 7 & -1.88 & 66.7 & $\mathrm{si} / \mathrm{no}$ \\
\hline \multirow{4}{*}{ IO-A8O } & 1 & -57.34 & 11.20 & $\mathrm{si} / \mathrm{si}$ \\
\hline & 3 & -56.33 & 12.21 & $\mathrm{si} / \mathrm{si}$ \\
\hline & 5 & -55.02 & 13.52 & $\mathrm{si} / \mathrm{si}$ \\
\hline & 7 & -4.88 & 63.66 & si/no \\
\hline \multirow{4}{*}{ IO-A7O } & 1 & -12.67 & 55.87 & $\mathrm{si} / \mathrm{no}$ \\
\hline & 3 & -3.57 & 64.97 & $\mathrm{si} / \mathrm{no}$ \\
\hline & 5 & -62.04 & 6.50 & $\mathrm{si} / \mathrm{si}$ \\
\hline & 7 & -45.90 & 22.64 & $\mathrm{si} / \mathrm{si}$ \\
\hline \multirow{4}{*}{ IO-A6O } & 1 & -1.64 & 66.90 & $\mathrm{si} / \mathrm{no}$ \\
\hline & 3 & -1.12 & 67.42 & si/no \\
\hline & 5 & -62.07 & 6.47 & $\mathrm{si} / \mathrm{si}$ \\
\hline & 7 & -53.54 & 15.00 & $\mathrm{si} / \mathrm{si}$ \\
\hline \multirow{4}{*}{$\mathrm{IO}-\mathrm{A} 3 \mathrm{O}$} & 1 & -59.12 & 9.42 & $\mathrm{si} / \mathrm{si}$ \\
\hline & 3 & -1.59 & 66.95 & si/no \\
\hline & 5 & 2.24 & 70.78 & si/no \\
\hline & 7 & -51.78 & 16.76 & $\mathrm{si} / \mathrm{si}$ \\
\hline \multirow[t]{4}{*}{ IO-A1O } & 1 & -2.23 & 66.31 & si/no \\
\hline & 3 & -56.34 & 12.20 & $\mathrm{si} / \mathrm{si}$ \\
\hline & 5 & 2.06 & 70.60 & $\mathrm{si} / \mathrm{no}$ \\
\hline & 7 & --- & --- & $*$ \\
\hline
\end{tabular}

\footnotetext{
* Problemas de convergencia no hay resultados.
} 
Por otro lado en la Tabla 5.3.2.2 se resumen los parámetros geométricos determinados para los diferentes sitios de interacción que favorecieron en la reducción de la molécula del óxido nitroso en $\mathrm{N}_{2}$ y $\mathrm{O}_{\text {ads. }}$.

Tabla 5.3.2.2 Parámetros geométricos de la interacción de $\mathrm{N}_{2} \mathrm{O}$ sobre átomos de Pt del isómero oxidado, IO-E25, por el átomo de $\mathrm{O}$ del $\mathrm{N}_{2} \mathrm{O}$. Multiplicidad (M), Frecuencias de vibración (v), distancias de enlace (d).

\begin{tabular}{|c|c|c|c|c|c|c|}
\hline $\begin{array}{c}\text { Tipo de } \\
\text { interacción }\end{array}$ & $\begin{array}{c}M \\
2 s+1\end{array}$ & $\begin{array}{l}\mathrm{v}^{\mathrm{N}-\mathrm{N}} \\
\mathrm{cm}^{-1}\end{array}$ & 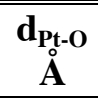 & $\begin{array}{c}\mathbf{d}_{\mathrm{Pt} \mathrm{N}} \\
\mathbf{A}\end{array}$ & $\begin{array}{l}\mathbf{d}_{\mathbf{P t}-\mathbf{P t}} \\
\mathbf{A}\end{array}$ & $\pm \underset{\AA}{\mathbf{A}}$ \\
\hline & $\overline{1} 1$ & 2268 & 2.49 & - & 2.62 & 0.08 \\
\hline \multirow[t]{4}{*}{ IO-A9O } & 3 & 2158 & 1.75 & 1.91 & 2.66 & 0.11 \\
\hline & 5 & 2167 & 1.76 & 1.95 & 2.67 & 0.14 \\
\hline & 7 & 1268 & - & 1.99 & 2.64 & 0.12 \\
\hline & 1 & 2192 & 1.75 & 1.93 & 2.62 & 0.08 \\
\hline \multirow[t]{4}{*}{ IO-A8O } & 3 & 2186 & 1.65 & 1.93 & 2.65 & 0.12 \\
\hline & 5 & 2186 & 1.75 & 1.93 & 2.67 & 0.14 \\
\hline & 7 & 1252 & - & 1.99 & 2.63 & 0.08 \\
\hline & 1 & 1782 & - & 2.08 & 2.62 & 0.08 \\
\hline \multirow[t]{4}{*}{ IO-A7O } & 3 & 2258 & 2.38 & - & 2.62 & 0.08 \\
\hline & 5 & 2172 & 1.76 & 1.91 & 2.65 & 0.12 \\
\hline & 7 & 2172 & 1.77 & 1.94 & 2.64 & 0.12 \\
\hline & 1 & 2270 & 2.82 & - & 2.62 & 0.08 \\
\hline \multirow[t]{4}{*}{$\mathrm{IO}-\mathrm{A} 6 \mathrm{O}$} & 3 & 2269 & 2.81 & - & 2.62 & 0.08 \\
\hline & 5 & 2176 & 1.76 & 1.92 & 2.64 & 0.10 \\
\hline & 7 & 2171 & 1.77 & 1.94 & 2.63 & 0.09 \\
\hline & 1 & 2157 & 1.76 & 1.88 & 2.65 & 0.12 \\
\hline \multirow[t]{3}{*}{$\mathrm{IO}-\mathrm{A} 3 \mathrm{O}$} & 3 & 2269 & 2.69 & - & 2.61 & 0.08 \\
\hline & 5 & 2269 & 2.63 & - & 2.61 & 0.08 \\
\hline & 7 & 2160 & 1.77 & 1.92 & 2.66 & 0.11 \\
\hline \multirow[t]{4}{*}{ IO-A1O } & 1 & 2269 & 2.63 & - & 2.62 & 0.08 \\
\hline & 3 & 2154 & 1.76 & 1.91 & 2.65 & 0.12 \\
\hline & 5 & 2271 & 2.64 & - & 2.61 & 0.08 \\
\hline & 7 & --- & --- & --- & --- & $*$ \\
\hline
\end{tabular}

\footnotetext{
* Problemas de convergencia no se muestran resultados.
} 
Los valores de frecuencias de vibración reportados son reales y máximos, $\sim 2100$ $\mathrm{cm}^{-1}$ cuando se presenta disociación y alrededor de 2200 y $1200 \mathrm{~cm}^{-1}$ cuando solo hay adsorción sin disociar. Estos valores son debidos a un modo normal de vibración, estiramiento del enlace N-N.

Con respecto a las distancias de enlace Pt-Pt, Pt-O y Pt-N para la interacción de más baja energía son: $2.65 \pm 0.12,1.76$ y $1.91 \AA$ respectivamente. En el resto de las interacciones la distancia Pt-Pt permanece en $\sim 2.60 \AA$. La presencia del $\mathrm{O}_{\text {ads }}$ sobre el isómero I favoreció la reactividad de esta con respecto a la del isómero sin oxidar. El átomo de $\mathrm{O}$ dona parte de su carga hacia el metal, distribuyéndola en todo el clúster y favoreciendo la disponibilidad de más e- que participen en la reacción. Aumentando la reactividad de la nanopartícula oxidada frente a la reducción de la molécula del óxido.

Interacciones por el átomo de $\mathrm{N}$ terminal también se favoreció solo en dos sitios. En la Figura 5.3.2.3 se muestran los resultados favorables de disociación del enlace NO con su valor correspondiente de multiplicidad de espín. La reacción solamente se favorece para multiplicidad de espín 5 en ambos acercamientos, en el resto de las multiplicidades se presenta adsorción no disociativa de la molécula del óxido. Los resultados se resumen en el anexo 9.1.6

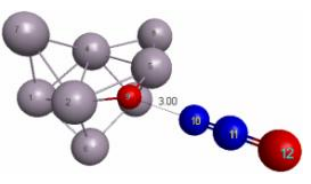

IO-A9N1

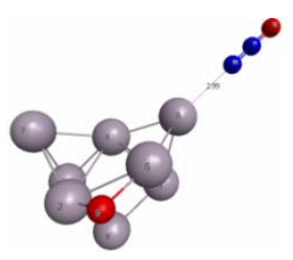

IO-A8N1

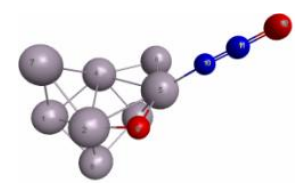

$M=1$

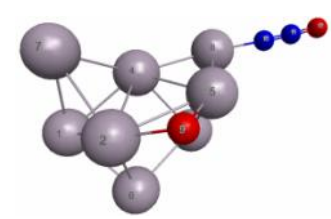

$M=1$

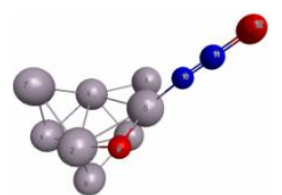

$M=3$

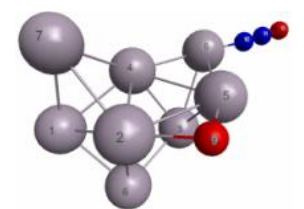

$M=3$

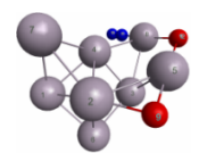

$M=5$

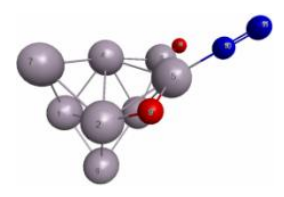

$M=5$

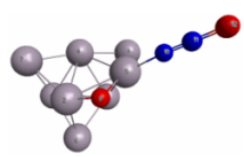

$M=7$

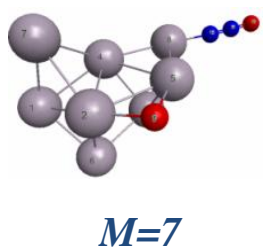

Figura 5.3.2.3 Resultados de interacción de $\mathrm{Pt}_{8} \mathrm{O}+\mathrm{N}_{2} \mathrm{O}$ en diferentes átomos $\mathrm{Pt}$ del isómero Ioxidado (IO-E25), por el lado del $N$ terminal, con distintas multiplicidades de espín $(M=2 s+1)$. 
Descripción de los acercamientos de la Figura 5.3.2.3

IO-A9N1: El átomo de $\mathrm{N}$ terminal del óxido se acerca directamente hacia el átomo de $\mathrm{O}_{\text {ads }}$ (sitio 9) del isómero oxidado, IO-E25, resultando disociación solo para multiplicidad de espín 5 con energía de $-58.17 \mathrm{kcal} / \mathrm{mol}$. Para el resto de las multiplicidades de espín solo se obtiene quimisorción, adsorbiendo la molécula del óxido por el $\mathrm{N}$ terminal. Los productos generados de la disociación se adsorben a la nanopartícula en sitios próximos al de interacción inicial.

IO-A8N1: La molécula del óxido se acerca isómero IO-E25, por el átomo de $\mathrm{N}$ terminal hacia el átomo ó sitio 8 del isómero, resultando en la reducción completa del óxido solo para multiplicidad de espín 5 , con una energía de $55.02 \mathrm{kcal} / \mathrm{mol}$. Para el resto de las multiplicidades solo hay adsorción sin disociar. Los productos de la reacción se adsorben en la nanopartícula en los sitios de interacción inicial y en átomos vecinos.

En estos dos acercamientos el de más baja energía es para IO-A9N1 con multiplicidad de espín 5. Este tipo de interacción con el átomo de $\mathrm{N}$ terminal acercando al átomo de Pt no es favorable, solo se presentaron dos casos con valores de multiplicidades de espín altos. El átomo de $\mathrm{N}$ terminal no es suficientemente atractor de los electrones disponibles en el metal para promover su transferencia, por lo que solo se presenta un número reducido de casos favorables para la reducción del óxido.

En la tabla 5.3.2.3 se reportan los parámetros energía determinados para este acercamiento, tipo de interacción, multiplicidad de espín, energías de disociación y relativas. El resto de las interacción que no favorables se resumen en el Anexo 9.1.6 
Tabla 5.3.2.3 Parámetros energéticos de la interacción del $\mathrm{N}_{2} \mathrm{O}$ sobre átomos del isómero oxidado, IOE25, por el átomo de $N$ terminal. Energías de disociación $\left(E_{d i s}\right)$ y energía relativa $\left(\mathbf{E}_{\text {relativa }}\right)$.

\begin{tabular}{ccccc}
\hline \hline $\begin{array}{c}\text { Tipo de } \\
\text { interacción }\end{array}$ & $\begin{array}{c}\mathbf{M} \\
\mathbf{2 s + 1}\end{array}$ & $\begin{array}{c}\mathbf{E}_{\mathbf{d i s}} \\
\mathbf{k c a l} / \mathbf{m o l}\end{array}$ & $\begin{array}{c}\mathbf{E}_{\text {relativa }} \\
\mathbf{k c a l} / \mathbf{m o l}\end{array}$ & $\begin{array}{c}\text { Enlace } \\
\text { /disociación }\end{array}$ \\
\hline \hline \multirow{2}{*}{ IO-A9N1 } & 1 & -14.15 & 54.13 & $\mathrm{si} / \mathrm{no}$ \\
& 3 & -17.89 & 50.69 & $\mathrm{si} / \mathrm{no}$ \\
& 5 & -58.17 & 10.41 & $\mathrm{si} / \mathrm{si}$ \\
& 7 & -11.71 & 56.83 & $\mathrm{si} / \mathrm{no}$ \\
\hline \multirow{2}{*}{ IO-A8N1 } & 1 & -14.13 & 54.41 & $\mathrm{si} / \mathrm{no}$ \\
& 3 & -12.07 & 56.47 & $\mathrm{si} / \mathrm{no}$ \\
& 5 & -55.05 & 13.49 & $\mathrm{si} / \mathrm{si}$ \\
& 7 & -4.87 & 63.67 & $\mathrm{si} / \mathrm{no}$ \\
\hline \hline
\end{tabular}

En la tabla 5.3.2.4 se reportan los parámetros geométricos determinados para este acercamiento, las frecuencias de vibración son todas reales y máximas. Su valor $\sim 2100$ $\mathrm{cm}^{-1}$ corresponde a un modo normal de vibración del enlace N-N. En el resto los valores disminuye debido a que no se presenta disociación de la molécula, el enlace N$\mathrm{N}$ no está libre de moverse por la presencia de otros átomos enlazados a estos.

Tabla 5.3.2.4 Parámetros geométricos de la interacción de $\mathrm{N}_{2} \mathrm{O}$ sobre átomos del isómero oxidado, IO-E25 por el átomo de $N$ terminal (N1). Multiplicidad de espín (M), frecuencias de vibración (v) y distancias de enlace (d).

\begin{tabular}{|c|c|c|c|c|c|c|}
\hline $\begin{array}{c}\text { Tipo de } \\
\text { interacción }\end{array}$ & $\begin{array}{c}M \\
2 s+1\end{array}$ & $\begin{array}{l}v^{N-N} \\
\mathrm{~cm}^{-1}\end{array}$ & $\begin{array}{c}\mathbf{d}_{\text {Pt-O }} \\
\AA\end{array}$ & $\underset{\AA}{\stackrel{d_{P t-N}}{\AA}}$ & $\underset{\AA}{\stackrel{\mathbf{d}_{\text {Pt-Pt }}}{\AA}}$ & $\underset{\stackrel{0}{\mathbf{\AA}}}{\mathbf{\mathbf { d }}}$ \\
\hline \multirow{4}{*}{ IO-A9N1 } & 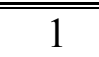 & 1257 & - & 1.98 & 2.63 & "0.09 \\
\hline & 3 & 1270 & - & 1.97 & 2.64 & 0.12 \\
\hline & 5 & 2172 & 1.91 & 1.90 & 2.62 & 0.08 \\
\hline & 7 & 1259 & - & 1.97 & 2.66 & 0.11 \\
\hline \multirow{4}{*}{ IO-A8N1 } & 1 & 1248 & - & 1.99 & 2.62 & 0.08 \\
\hline & 3 & 1247 & - & 2.00 & 2.62 & 0.08 \\
\hline & 5 & 2186 & 1.75 & 1.93 & 2.67 & 0.14 \\
\hline & 7 & 1252 & - & 1.99 & 2.63 & 0.09 \\
\hline
\end{tabular}


Las distancias Pt-O, Pt-N y Pt-Pt son $\sim 1.75, \sim 1.93$ y $\sim 2.67 \AA$ respectivamente para los casos de disociación. El enlace formado entre $\mathrm{Pt}$ con $\mathrm{O}$ es más corto que el de Pt con $\mathrm{N}$, lo que significa que el átomo de oxígeno está unido al metal con mayor fuerza.

En general estos nuevos resultados se favorecieron por la presencia del oxígeno adsorbido en la superficie del isómero I, el átomo de $\mathrm{O}$ genera una mayor reactividad frente a la reducción del óxido, se obtiene un mayor número de sitios reactivos comparado con los obtenidos para el isómero sin oxidar. Estos nuevos sitios se favorecen por la distribución de carga del átomo de $\mathrm{O}$ en otros átomos del cúmulo. Un análisis de cargas de los átomos se puede ver más adelante.

Además se calcula el porcentaje de deformación de la estructura de la nanopartícula oxidada después de la interacción, los resultados obtenidos demuestran que la deformación es menor al 3\%, lo que significa que la estructura geométrica se conserva.

\section{Interacción por enlaces.}

En esta segunda parte se presentan los resultados de la interacción $\mathrm{Pt}_{8} \mathrm{O}+\mathrm{N}_{2} \mathrm{O}$, acercando la molécula del óxido directamente a enlaces Pt-Pt del isómero oxidado. Los acercamientos favorables se ilustran a continuación.

En la Figura 5.3.2.4 se ilustran las interacciones iníciales en los sitios favorables donde se obtiene reducción de la molécula del $\mathrm{N}_{2} \mathrm{O}$. Además se incluye la geometría final que adquieren los productos después de la reacción a cada multiplicidad de espín (M). 

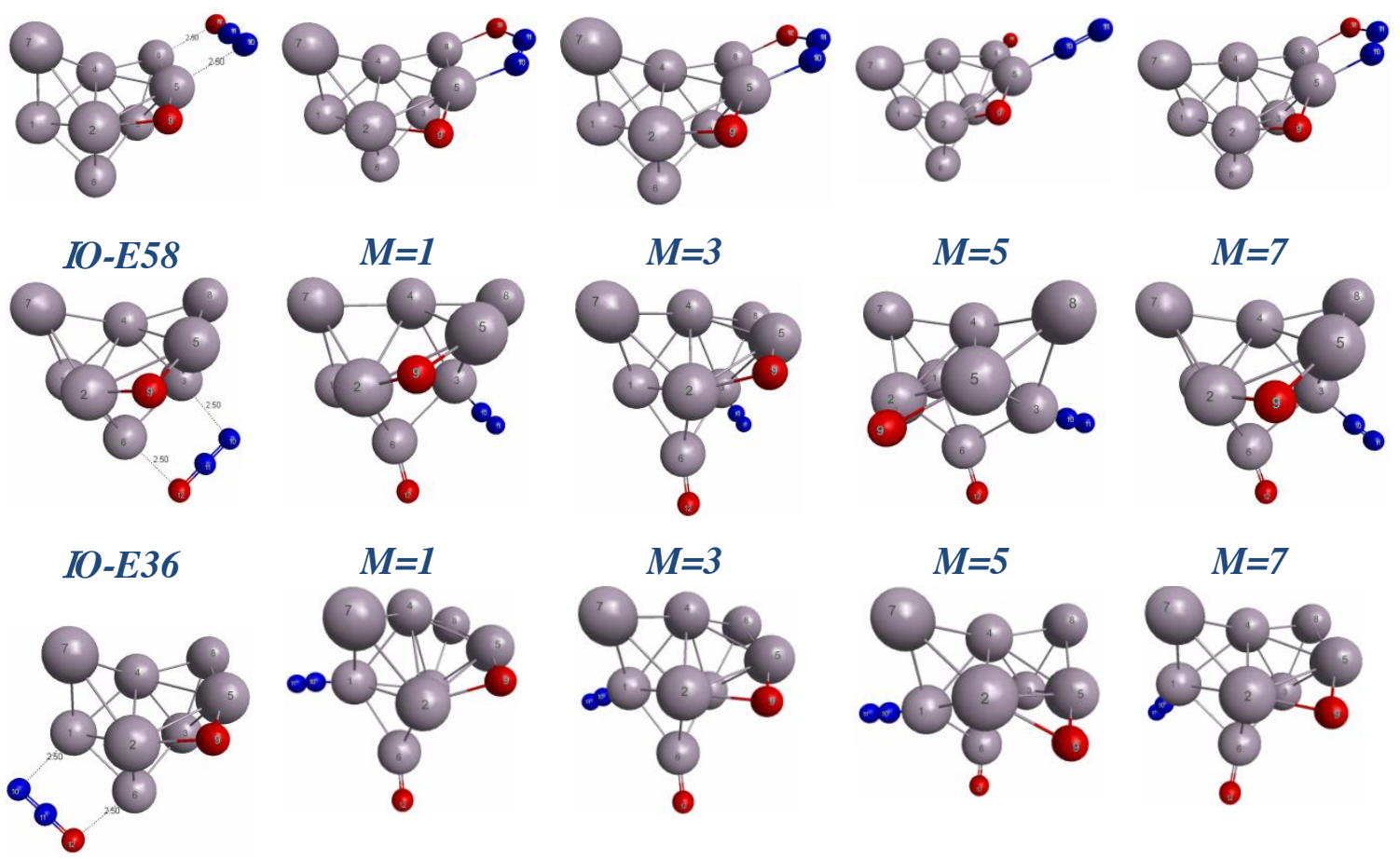

IO-E16

$M=1$

$M=3$

$M=5$

$M=7$

Figura 5.3.2.4 Resultados de interacción de Pt $t_{8} \mathrm{O}+\mathrm{N}_{2} \mathrm{O}$ en diferentes enlaces Pt-Pt del isómero I oxidado, IO-E25, con distintas multiplicidades de espín 1, 3, 5 y 7 $(M=2 s+1)$.

Los diferentes tipos de interacciones se etiquetaron de la siguiente manera. Ej. Para la interacción IO-E58, 1er termino "I" por isómero I, 2do termino "O" por oxidación, 3er termino "E" por la interacción en enlaces Pt-Pt, finalmente "58" sitio de interacción en el isómero oxidado.

Descripción de acercamientos en la Figura 5.3.2.4

IO-E58: La molécula de $\mathrm{N}_{2} \mathrm{O}$ se acerca paralela al enlace de Pt-Pt, interactúa con los átomos 5 y 8 , la reducción se presenta solo en multiplicidad de espín 5 con una energía de $-55.04 \mathrm{kcal} / \mathrm{mol}$. En el resto de las multiplicidades el $\mathrm{N}_{2} \mathrm{O}$ se adsorbe por sus extremos formando un enlace tipo puenteado, las energías van de $\sim-5$ hasta $\sim-14 \mathrm{kcal} / \mathrm{mol}$. Las distancias promedio Pt-Pt varían de 2.62-2.67 A, y las distancias Pt-O y Pt-N se acortan cuando hay rompimiento 
de la molécula. Los productos de reacción se adsorben a la nanopartícula en los átomos iníciales de interacción.

IO-E36: Acercando la molécula de $\mathrm{N}_{2} \mathrm{O}$ a los átomos 3 y 6 del isómero IO-E58 se reduce por completo en $\mathrm{N}_{2}$ y $\mathrm{O}$ en todos los valores de multiplicidad de espín calculados $(1,3,5$ y 7). Las energías de disociación van desde -53 a -63 $\mathrm{kcal} / \mathrm{mol}$. El de más baja energía se encuentra en multiplicidad de espín 3. Las

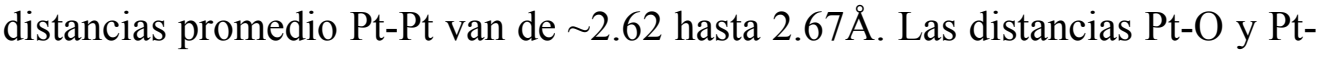
$\mathrm{N}$ no varían mucho son casi constantes en todas las multiplicidades. Los productos de reacción se adsorben en el isómero oxidado.

IO-E16: Acercando el $\mathrm{N}_{2} \mathrm{O}$ a los átomos 1 y 6 del isómero IO-E58, se obtiene su reducción para multiplicidad de espín 1, 3, 5 y 7. Los valores de la energía de disociación van desde $\sim-47$ hasta las $61 \mathrm{kcal} / \mathrm{mol}$. Las distancias Pt-Pt varían desde los 2.60 a $2.67 \AA$ y las distancias Pt-O y Pt-N se mantienen prácticamente sin cambios importantes, entres los 1.75 y $1.95 \AA$ respectivamente. Al igual que en los casos anteriores los productos de reacción son adsorbidos en la nanopartícula en los átomos iníciales de interacción.

En la Tabla 5.3.2.5 se muestran los resultados energéticos de estos acercamientos que favorece la disociación de óxido nitroso, con su multiplicidad de espín correspondiente. La energía más baja determinada es de $-63.59 \mathrm{kcal} / \mathrm{mol}$ para el acercamiento IO-E36 con multiplicad de espín 3. Este valor esta degenerado para la M 1 y 5, con 1.52 y 2.16 $\mathrm{kcal} / \mathrm{mol}$ respectivamente, y para la interacción IO-E16 con multiplicidad de espín 3 con $1.69 \mathrm{kcal} / \mathrm{mol}$. 
Tabla 5.3.2.5 Parámetros energéticos de la interacción de $\mathrm{N}_{2} \mathrm{O}$ en enlaces Pt-Pt del isómero oxidado, IO-E25. Energías de disociación $\left(E_{\text {dis }}\right)$ y energía relativa $\left(E_{\text {relativa }}\right)$.

\begin{tabular}{ccccc}
\hline \hline $\begin{array}{c}\text { Tipo de } \\
\text { interacción }\end{array}$ & $\begin{array}{c}\mathbf{M} \\
\mathbf{2 s + 1}\end{array}$ & $\begin{array}{c}\mathbf{E}_{\mathbf{d i s}} \\
\mathbf{k c a l} / \mathbf{m o l}\end{array}$ & $\begin{array}{c}\mathbf{E}_{\mathbf{r e l a t i v a}} \\
\mathbf{k c a l} / \mathbf{m o l}\end{array}$ & $\begin{array}{c}\text { Enlace/ } \\
\text { disociación }\end{array}$ \\
\hline \hline \multirow{3}{*}{ IO-E58 } & 1 & -14.26 & 49.33 & $\mathrm{si} / \mathrm{no}$ \\
& 3 & -13.67 & 49.92 & $\mathrm{si} / \mathrm{no}$ \\
& 5 & -55.04 & 8.55 & $\mathrm{si} / \mathrm{si}$ \\
& 7 & -5.38 & 58.21 & $\mathrm{si} / \mathrm{no}$ \\
\hline \multirow{2}{*}{ IO-E36 } & 1 & -61.43 & 2.16 & $\mathrm{si} / \mathrm{si}$ \\
& 3 & -63.59 & 0.00 & $\mathrm{si} / \mathrm{si}$ \\
& 5 & -62.07 & 1.52 & $\mathrm{si} / \mathrm{si}$ \\
& 7 & -53.47 & 10.12 & $\mathrm{si} / \mathrm{si}$ \\
\hline \multirow{2}{*}{ IO-E16 } & 1 & -61.43 & 2.16 & $\mathrm{si} / \mathrm{si}$ \\
& 3 & -61.90 & 1.69 & $\mathrm{si} / \mathrm{si}$ \\
& 5 & -60.46 & 3.13 & $\mathrm{si} / \mathrm{si}$ \\
& 7 & -47.57 & 16.02 & $\mathrm{si} / \mathrm{si}$ \\
\hline \hline
\end{tabular}

En la Tabla 5.3.2.6 se resumen los parámetros geométricos de estos acercamientos que se favorecen a la disociación de óxido nitroso, con su multiplicidad de espín correspondiente. Las frecuencias de vibración son reales y se reporta su valor máximo en todas las interacciones estudiadas, su valor $\sim 2100 \mathrm{~cm}^{-1}$ en el caso de adsorción disociativa $\mathrm{y} \sim 1200-1600 \mathrm{~cm}^{-1}$ en adsorción sin disociar, corresponden al movimiento "stretching" de la molécula de N-N. Otras interacciones se muestran en el Anexo 9.1.6 
Tabla 5.3.2.6 Parámetros geométricos para la interacción de $\mathrm{N}_{2} \mathrm{O}$ en enlaces Pt-Pt del isómero oxidado, IO-E25. Multiplicidad de espín (M), frecuencias de vibración (v), distancias de enlace $(d)$.

\begin{tabular}{|c|c|c|c|c|c|c|}
\hline $\begin{array}{c}\text { Tipo de } \\
\text { interacción }\end{array}$ & $\begin{array}{c}M \\
2 s+1\end{array}$ & $\mathrm{~cm}^{-1}$ & $\begin{array}{c}\mathbf{d}_{\text {Pt-O }} \\
\stackrel{\AA}{\mathbf{A}}\end{array}$ & $\begin{array}{c}\mathbf{d}_{\mathbf{P t}-\mathrm{N}} \\
\AA \\
\AA\end{array}$ & $\begin{array}{c}\mathbf{d}_{\mathbf{P t}-\mathbf{P t}} \\
\AA\end{array}$ & $\begin{array}{c} \pm \mathbf{d}_{\mathbf{P t}-\mathbf{P t}} \\
\AA\end{array}$ \\
\hline \multirow{4}{*}{ IO-E58 } & 1 & 1635 & 2.03 & 1.99 & 2.62 & 0.08 \\
\hline & 3 & 1627 & 2.02 & 1.98 & 2.62 & 0.08 \\
\hline & 5 & 2185 & 1.75 & 1.93 & 2.67 & 0.12 \\
\hline & 7 & 1626 & 2.06 & 1.98 & 2.65 & 0.14 \\
\hline \multirow{4}{*}{ IO-E36 } & 1 & 2172 & 1.75 & 1.92 & 2.62 & 0.08 \\
\hline & 3 & 2169 & 1.76 & 1.91 & 2.67 & 0.14 \\
\hline & 5 & 2176 & 1.76 & 1.92 & 2.62 & 0.08 \\
\hline & 7 & 2171 & 1.77 & 1.94 & 2.63 & 0.09 \\
\hline \multirow{4}{*}{ IO-E16 } & 1 & 2169 & 1.75 & 1.96 & 2.67 & 0.14 \\
\hline & 3 & 2168 & 1.75 & 1.95 & 2.66 & 0.11 \\
\hline & 5 & 2178 & 1.75 & 1.93 & 2.60 & 0.09 \\
\hline & 7 & 2167 & 1.76 & 1.94 & 2.65 & 0.12 \\
\hline
\end{tabular}

La distancia promedio Pt-Pt de la nanopartícula reportada para la interacción de más baja energía es de $2.67 \pm 0.14 \AA$, en el resto de los acercamientos estas distancias varían $2.65 \AA$. Los átomos de $\mathrm{O}$ y $\mathrm{N}$ se enlazan a un átomo de Pt diferente de la nanopartícula a una distancia de 1.75 y $1.98 \AA$ respectivamente.

De manera general de todos los acercamientos probados, los más favorables a la reducción de la molécula del $\mathrm{N}_{2} \mathrm{O}$ fueron aquellos donde la molécula de $\mathrm{N}_{2} \mathrm{Ose}$ acerca directamente con los enlaces Pt-Pt, ambos paralelos, generando solo tres sitios reactivos, estos son: IO-E58, IO-E36 y IO-E16 estos últimos dos fueron reactivos a la molécula del óxido para todos los valores de multiplicidad de espín calculados, 1, 3 , 5 y 7.

Las características que presentaron estos átomos que fueron reactivos al $\mathrm{N}_{2} \mathrm{O}$, es principalmente la carga inicial, pues los sitios más reactivos, IO-E36 y IO-E16, están formados por átomos carga opuesta pero con valores pequeños, el resto de los sitios no mostraron reactividad, ya que no cumplen con estas características. 
Además de la reducción de este óxido se obtiene dos diferentes tipos de adsorción sin disociar del $\mathrm{N}_{2} \mathrm{O}$ en la nanopartícula: primero con adsorción por el $\mathrm{N}$ terminal (Ver anexo 9.5) y segundo con adsorción por los extremos de la molécula, formando enlaces tipo puente (Figura 5.3.2.4, IO-E58 con M 1, 3 y 7).

\subsubsection{Análisis de cargas Hirshfeld de la reacción de $\mathrm{N}_{2} \mathrm{O}$ sobre $\mathrm{Pt}_{8} \mathrm{O}$.}

Como se menciono anteriormente el esquema de Hirshfeld es de los más empleados y más confiable para determinar la distribución de carga en una molécula, por lo cual en este trabajo se empleo para calcular la distribución de carga en cada sistema. El análisis de las cargas de Hirshfeld en las interacciones de $\mathrm{N}_{2} \mathrm{O}$ con $\mathrm{Pt}_{8} \mathrm{O}$ antes y después de la interacción se realizó solo para casos favorables de rompimiento del enlace N-O en la molécula del óxido nitroso, como a continuación se ilustra en la Figura 5.3.3.1. Este análisis ayudó a predecir el comportamiento del metal en la reducción del $\mathrm{N}_{2} \mathrm{O}$.

En la Figura 5.3.3.1 se resumen las interacciones que favorecieron la disociación de la molécula del $\mathrm{N}_{2} \mathrm{O}$ en la superficie del Pt, acercando por los extremos de la molécula de óxido a un átomo (sitio reactivo) de la superficie de la nanopartícula.

En la Tabla 5.3.3.1 se muestran los valores de las cargas iníciales $\left(\mathrm{q}_{\mathrm{i}}\right)$ de los átomos antes de la interacción, cargas finales $\left(\mathrm{q}_{\mathrm{f}}\right)$ de todos los átomos después de realizar la interacción del $\mathrm{N}_{2} \mathrm{O}$ en el platino y finalmente se calculan sus cambios finales $(\Delta \mathrm{q})$. Los valores de las cargas que se reportan para cada interacción solo son para un valor de multiplicidad de espín.. Con este análisis de cargas se determinó que los cambios más importantes es entre átomos que participan directamente en la interacción, principalmente los átomos del clúster de platino son los responsables de donar carga hacia la molécula del óxido. 


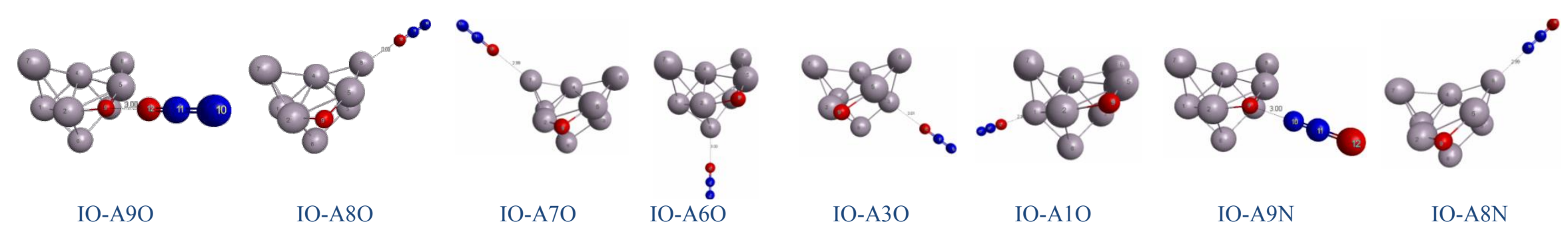

Figura 5.3.3.1 Interacciones de $\mathrm{N}_{2} \mathrm{O}$ con resultados favorables de disociación. Acercamientos directos por el O del óxido hacia un átomo de Pt.

Tabla 5.3.3.1 Análisis de cargas Hirshfeld de las interacciones del $\mathrm{N}_{2} \mathrm{O}$ hacia el isómero oxidado, IO-E25, con acercamientos hacia átomos por el lado del $O$ del óxido. Cargas iníciales $\left(q_{i}\right)$, cargas finales $\left(q_{f}\right)$ y cambio total de carga $(\Delta q)$ para todos los átomos.

\begin{tabular}{|c|c|c|c|c|c|c|c|c|c|c|c|c|c|c|c|c|c|}
\hline \multicolumn{2}{|c|}{ Átomos } & \multicolumn{2}{|c|}{${ }^{3} I O-A 9 O$} & \multicolumn{2}{|c|}{${ }^{1} \underline{I O}-A 8 O$} & \multicolumn{2}{|c|}{$\underline{{ }^{5} \mathrm{IO}-\mathrm{A} 7 \mathrm{O}}$} & \multicolumn{2}{|c|}{ IIO-A6O } & \multicolumn{2}{|c|}{ 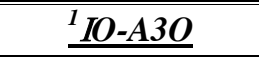 } & \multicolumn{2}{|c|}{$\underline{{ }^{3} I O-A 1 O}$} & \multicolumn{2}{|c|}{$\underline{{ }^{5} I O-A 9 N}$} & \multicolumn{2}{|c|}{${ }^{5} I O-A 8 N$} \\
\hline & $\boldsymbol{q}_{i}$ & $q_{f}$ & $\Delta q$ & $q_{f}$ & $\Delta q$ & $q_{f}$ & $\Delta q$ & $\boldsymbol{q}_{f}$ & $\Delta q$ & $\boldsymbol{q}_{f}$ & $\Delta q$ & $q_{f}$ & $\Delta q$ & $\boldsymbol{q}_{f}$ & $\Delta q$ & $q_{f}$ & $\Delta q$ \\
\hline $1 \mathrm{Pt}$ & 0.002 & 0.029 & 0.027 & -0.006 & $\begin{array}{c}-0.008 \\
\end{array}$ & 0.037 & 0.035 & 0.003 & 0.001 & 0.020 & 0.018 & 0.178 & $\underline{0.176}$ & 0.018 & 0.016 & 0.041 & 0.002 \\
\hline $2 \mathrm{Pt}$ & 0.138 & 0.129 & -0.009 & 0.147 & 0.008 & 0.140 & 0.002 & 0.151 & 0.013 & 0.154 & 0.016 & 0.124 & -0.014 & 0.144 & 0.006 & 0.135 & $\underline{0.138}$ \\
\hline $3 \mathrm{Pt}$ & 0.010 & -0.002 & -0.012 & -0.009 & -0.019 & 0.000 & -0.010 & 0.072 & 0.062 & 0.171 & $\underline{0.161}$ & 0.031 & 0.021 & 0.026 & 0.016 & 0.021 & 0.010 \\
\hline $4 \mathrm{Pt}$ & 0.017 & 0.016 & -0.001 & 0.010 & -0.007 & 0.073 & 0.056 & 0.002 & -0.015 & 0.012 & -0.005 & 0.016 & -0.001 & 0.009 & -0.008 & 0.014 & 0.017 \\
\hline $5 \mathrm{Pt}$ & 0.157 & 0.144 & -0.013 & 0.158 & 0.001 & 0.113 & -0.044 & 0.118 & -0.039 & 0.097 & -0.060 & 0.135 & -0.022 & 0.227 & 0.070 & 0.143 & $\underline{0.157}$ \\
\hline $6 \mathrm{Pt}$ & -0.024 & 0.011 & 0.035 & 0.027 & 0.051 & 0.003 & 0.027 & 0.170 & $\underline{0.194}$ & 0.038 & 0.062 & 0.032 & 0.056 & -0.005 & 0.019 & -0.018 & -0.024 \\
\hline $7 \mathrm{Pt}$ & -0.016 & 0.152 & $\underline{0.168}$ & 0.020 & 0.036 & 0.153 & $\underline{0.169}$ & 0.013 & 0.029 & 0.008 & 0.024 & 0.042 & 0.058 & -0.005 & 0.011 & 0.002 & -0.016 \\
\hline $8 \mathrm{Pt}$ & -0.040 & 0.041 & 0.081 & 0.147 & $\underline{0.187}$ & -0.011 & 0.029 & -0.008 & 0.032 & 0.028 & 0.068 & -0.018 & 0.022 & 0.161 & $\underline{0.201}$ & 0.158 & -0.040 \\
\hline 90 & -0.244 & -0.256 & -0.012 & -0.246 & -0.002 & -0.249 & -0.005 & -0.268 & -0.024 & -0.243 & 0.001 & -0.261 & -0.017 & -0.257 & -0.013 & -0.251 & $\underline{-0.244}$ \\
\hline $10 \mathrm{O}$ & -0.112 & -0.262 & $\underline{-0.150}$ & -0.278 & $\underline{-0.166}$ & -0.259 & $\underline{-0.147}$ & -0.258 & $\underline{-0.146}$ & -0.257 & $\underline{-0.145}$ & -0.258 & $\underline{-0.146}$ & -0.291 & $\underline{-0.179}$ & -0.272 & $\underline{-0.112}$ \\
\hline $11 \mathrm{~N}$ & 0.196 & 0.008 & $\underline{-0.188}$ & 0.004 & -0.193 & -0.012 & -0.208 & 0.013 & -0.183 & -0.031 & -0.227 & 0.002 & -0.194 & -0.024 & -0.220 & 0.024 & $\underline{0.196}$ \\
\hline $12 \mathrm{~N}$ & -0.084 & -0.009 & 0.093 & 0.027 & 0.111 & 0.012 & 0.096 & -0.009 & 0.075 & 0.004 & 0.088 & -0.023 & 0.062 & -0.003 & 0.081 & 0.003 & -0.084 \\
\hline
\end{tabular}

(-) Átomos con cambios significativos $\Delta q>0$, carga positiva.

(-) Átomos con cambios significativos $\Delta q<0$, carga negativa. 
Como se puede ver en la Tabla 5.3.3.1, los $\Delta \mathrm{q}>0$ en color azul son los átomos que donan carga, quedando deficientes de e-, y los átomos con $\Delta \mathrm{q}<0$ son los que la aceptan (color rojo). Este comportamiento se observa para todas las interacciones reportadas. En el caso donde no se presenta disociación de la molécula del óxido los valores de $\Delta \mathrm{q} \sim 0$ es decir no se presentan cambios importantes en sus cargas.

En general para que este tipo de sitios sea reactivo al $\mathrm{N}_{2} \mathrm{O}$ el átomo debe tener carga negativa suficientemente grande para que haya transferencia de electrones del átomo del metal hacia la molécula del óxido. Adicional a tabla 5.3.3.1, se determino el análisis de cargas ahora para otro tipo de interacción, ahora acercamientos por enlaces. Los casos más favorables se ilustran en la Figura 5.3.3.2 que dieron lugar a la disociación de la molécula del $\mathrm{N}_{2} \mathrm{O}$, acercándola paralela a un enlace (sitio reactivo) de la superficie de la nanopartícula.

En la Tabla 5.3.3.2 se reportan los valores de carga inicial $\left(\mathrm{q}_{\mathrm{i}}\right)$ de los átomos antes de la reacción, cargas finales $\left(\mathrm{q}_{\mathrm{f}}\right)$ después de la reacción del $\mathrm{N}_{2} \mathrm{O}$ en el platino y finalmente se calculan los cambios de carga que sufre estos átomos $(\Delta q)$. Los valores de las cargas que se reportan en cada interacción corresponden para un solo valor de multiplicidad de espín.

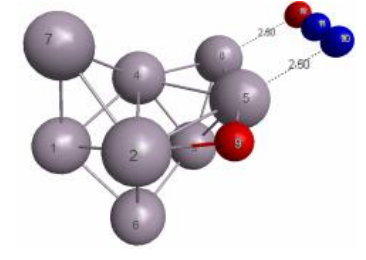

IO-E58

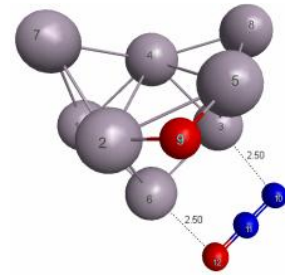

IO-E36

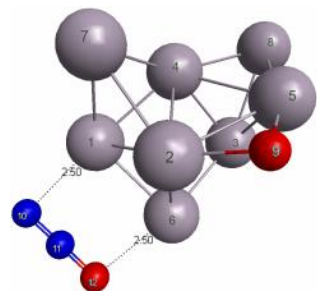

IO-E16

Figura 5.3.3.2 Interacciones de $\mathrm{N}_{2} \mathrm{O}$ con resultados favorables de disociación. Acercamientos hacia enlaces paralelos al eje de la molécula del óxido.

De igual manera al caso anterior, con este análisis de cargas se determinó que los cambios más importantes son aquellos donde se están llevando a cabo la reacción es entre los átomos que participan directamente en la interacción, cargas color azul donan e- y cargas 
color rojo aceptan e-, los átomos del platino que poseen carga $<0$, son los responsables de entregarla hacia la molécula del óxido para favorecer el rompimiento del enlace N-O.

Tabla 5.3.3.2 Análisis de cargas Hirshfeld de las interacciones del $\mathrm{N}_{2} \mathrm{O}$ hacia el isómero oxidado, IO-E25, con acercamientos hacia enlaces. Cargas iníciales $\left(q_{i}\right)$, cargas finales $\left(q_{f}\right)$ y cambios totales de carga $(\Delta q)$ para todos los átomos.

\begin{tabular}{|c|c|c|c|c|c|c|c|}
\hline \multirow[b]{2}{*}{ Átomos } & \multirow[b]{2}{*}{$q_{i}$} & \multicolumn{2}{|c|}{ ㅍ-E58 } & \multicolumn{2}{|c|}{${ }^{3} I O-E 36$} & \multicolumn{2}{|c|}{${ }^{3} I O-E 16$} \\
\hline & & $\boldsymbol{q}_{f}$ & $\Delta q$ & $q_{f}$ & $\Delta q$ & $\boldsymbol{q}_{f}$ & $\Delta q$ \\
\hline 11 $\mathrm{Pt}$ & 0.002 & 0.042 & 0.040 & 0.014 & 0.012 & 0.043 & 0.041 \\
\hline $2 \mathrm{Pt}$ & 0.138 & 0.1355 & -0.003 & 0.132 & -0.006 & 0.131 & -0.007 \\
\hline $3 \mathrm{Pt}$ & 0.010 & 0.0206 & 0.011 & 0.072 & 0.062 & 0.028 & 0.018 \\
\hline $4 \mathrm{Pt}$ & 0.017 & 0.015 & -0.002 & 0.014 & -0.003 & 0.016 & -0.001 \\
\hline $5 \mathrm{Pt}$ & 0.157 & 0.1426 & -0.014 & 0.131 & -0.026 & 0.154 & -0.003 \\
\hline $6 \mathrm{Pt}$ & -0.024 & -0.0174 & 0.007 & 0.151 & 0.175 & 0.167 & 0.191 \\
\hline $7 \mathrm{Pt}$ & -0.016 & -0.001 & 0.015 & 0.013 & 0.029 & -0.016 & 0.000 \\
\hline $8 \mathrm{Pt}$ & -0.040 & 0.1578 & 0.198 & -0.014 & 0.026 & -0.015 & 0.025 \\
\hline $9 \mathrm{O}$ & -0.244 & -0.2509 & -0.007 & -0.250 & -0.006 & -0.251 & -0.007 \\
\hline $10 \mathrm{O}$ & -0.112 & -0.2718 & -0.160 & -0.263 & $\underline{-0.151}$ & -0.247 & $\underline{-0.135}$ \\
\hline $11 \mathrm{~N}$ & 0.196 & 0.0239 & $\overline{-0.172}$ & 0.012 & -0.184 & 0.004 & -0.192 \\
\hline $12 \mathrm{~N}$ & -0.084 & 0.0036 & 0.088 & -0.013 & 0.071 & -0.014 & 0.070 \\
\hline
\end{tabular}

$(-)$ Átomos con cambios significativos $\Delta q>0$, carga positiva.

(-) Átomos con cambios significativos $\Delta q<0$, carga negativa.

Para favorecer el rompimiento del enlace N-O, el sitio reactivo formado por dos átomo de Pt de cumplir con ciertas características, una de ellas es que sus átomos deben poseer cargas opuestas y pequeñas, de lo contrario la transferencia de electrones se dificulta, el átomo con carga negativa es quien dona la carga.

De manera general los principales parámetros que determinan la reactividad de la nanopartícula con la molécula de óxido son las cargas y las multiplicidades de espín. Los casos de disociación se favoreció cuando las cargas más grandes y negativas (mayor numero de electrones) se localizan en los átomos.

El mecanismo de disociación es el mismo en todos los casos, los átomos de Pt principales donadores de carga, la transfieren al átomo de $\mathrm{O}$ del $\mathrm{N}_{2} \mathrm{O}$ debilitando el enlace $\mathrm{O}-\mathrm{N}$ hasta que finalmente se rompe, quedando adsorbidos ambos productos de la reacción en la 
nanopartícula, como sucede en todos los casos estudiados. El resto de los átomos no muestran cambios significativos. 


\section{Conclusiones}

En este estudio se logra determinar la disociación de la molécula de $\mathrm{N}_{2} \mathrm{O}$ sobre Pt, un metal menos caro que $\mathrm{Rh}$, empleando nanopartículas de ocho átomos de platino en estado neutro, $\mathrm{Pt}_{8}$ (único trabajo), otros autores empelan iones de este metal, y también al doparlas con oxígeno, $\mathrm{Pt}_{8} \mathrm{O}$, el rendimiento aumenta. Los orbitales d son los responsables de la actividad catalítica en este metal.

La reacción de disociación: $\mathrm{N}_{2} \mathrm{O} \rightarrow \mathrm{N}_{2}+\mathrm{O}$, catalizada por nanopartículas de $\mathrm{Pt}_{8}$ neutras es favorable $\left(\mathrm{E}_{\mathrm{dis}}<0 \mathrm{kcal} / \mathrm{mol}\right)$, como se formuló en la hipótesis. Las energías de disociación del enlace $\mathrm{N}-\mathrm{O}$ son de alrededor $\sim 60 \mathrm{kcal} / \mathrm{mol}$. Nunca se presenta rompimiento de enlace $\mathrm{N}-\mathrm{N}$ como es de esperarse (enlace más fuerte en la molécula de $\mathrm{N}_{2} \mathrm{O}$ ). Sin embargo los resultados determinados muestran poca eficiencia en la disociación de la molécula de $\mathrm{N}_{2} \mathrm{O}$, se determinaron pocos sitios reactivos (20 en total). En su mayoría se determinaron solo adsorciones no disociativas sobre la nanopartícula. La mayoría de las disociaciones se presentaron aproximando $\mathrm{N}_{2} \mathrm{O}$ paralelo a un enlace Pt-Pt del cúmulo, de esta manera se maximiza la interacción de los orbitales de frontera de las especies reactantes. También se obtuvo la ruptura al aproximar $\mathrm{N}_{2} \mathrm{O}$ al átomo de $\mathrm{Pt}$ con mayor carga negativa (único caso átomo 8), lo que no sucede con otros metales como Rh y Pd, que solo presentan reactividad en enlaces y caras.

La eficiencia se mejoró incorporando oxígeno a la nanopartícula, $\mathrm{Pt}_{8} \mathrm{O}$, como se planteo en la hipótesis, la presencia de este átomo adicional favorece su reactividad, se determinó un mayor número de casos favorables de rompimiento del enlace N-O (46 en total). Se puede ver claramente que la presencia del oxígeno mejora su reactividad. Las energías de disociación de enlace $\mathrm{N}-\mathrm{O}$ son $\sim 63 \mathrm{kcal} / \mathrm{mol}$. Al igual que en el caso anterior, para que se presente disociación, la molécula de $\mathrm{N}_{2} \mathrm{O}$ debe aproximarse paralelo a enlaces Pt-Pt, además acercamientos hacia átomos directamente ahora son más favorables.

En esta reacción catalizada por ambas nanopartículas $\mathrm{Pt}_{8}$ y $\mathrm{Pt}_{8} \mathrm{O}$ los factores que favorecen la disociación del $\mathrm{N}_{2} \mathrm{O}$ son los siguientes: (1) Naturaleza geométrica del sitio reactivo. (2) Geometría de interacción. (3) Estados electrónicos del metal (multiplicidad de espín). 
Además de los factores mencionados anteriormente, la reactividad en ambas nanopartículas se favorece por el tamaño y la forma que presentan, los átomos que conforman su geometría posee enlaces insaturados (todos los átomos son superficiales), por lo que $\mathrm{Pt}_{8}$ y $\mathrm{Pt}_{8} \mathrm{O}$ son más reactivas que una superficie del cristal de este mismo metal, $\mathrm{Pt}$ (111).

En un análisis de cargas empleando el esquema de Hirshfeld se determina transferencia de carga del metal hacia la molécula del $\mathrm{N}_{2} \mathrm{O}$ favoreciendo y promoviendo la disociación del enlace $\mathrm{N}-\mathrm{O}$ en la molécula del $\mathrm{N}_{2} \mathrm{O}$. Los estados excitados (multiplicidad de espín) del metal favorecen la transferencia de electrones.

Las energías de enlace obtenidas para $\mathrm{Pt}_{2}$ con $\mathrm{ADF}$ y para los cúmulos de platino son más grandes que los valores experimentales y que los obtenidos por otros autores aplicando otros métodos. Este resultado es una característica del método empleado, porque también se obtuvo en estudios con cúmulos de $\mathrm{Rh}$ realizados por Avilés et. al [44].

El posible mecanismo que sigue esta reacción se puede resumir en los siguientes pasos: (1) Alargamiento del enlace $\mathrm{N}-\mathrm{O}$ favorecido por la transferencia de carga que se genera del metal hacia la molécula. (2) Debilitamiento del enlace N-O y (3) Disociación de este enlace formando como productos, $\mathrm{N}_{2}$ y $\mathrm{O}$, adsorbidos sobre ambas estructuras de las nanopartícula $\mathrm{Pt}_{8}$ y $\mathrm{Pt}_{8} \mathrm{O}$. La adsorción del $\mathrm{N}_{2}$ es también un resultado característico del método empleado. Experimentalmente en las primeras disociaciones el $\mathrm{N}_{2}$ se desprende del catalizador. 


\section{Perspectivas}

Las siguientes alternativas que se proponen en la continuación de este trabajo comprenden las siguientes líneas de investigación:

1. Estudio de la adsorción disociativa de $\mathrm{N}_{2} \mathrm{O}$ en las nanoparticulas con carga $\mathrm{de}_{\mathrm{Pt}_{8}}{ }^{\mathrm{y}} \mathrm{y}$ $\mathrm{Pt}_{8} \mathrm{O}^{ \pm}$. Para determinar la reactividad de estas frente a la disociacion de la molecula de óxido.

2. Caracterización de la superficie TiO2 (101) anatasa con vacancias de oxígeno.

3. Estudiar la adsorcion de las nanoparticulas con carga y sin carga de $\mathrm{Pt}_{8}, \mathrm{Pt}_{8}{ }^{ \pm}, \mathrm{Pt}_{8} \mathrm{O}$ y $\mathrm{Pt}_{8} \mathrm{O}^{ \pm}$sobre la superficie $\mathrm{TiO}_{2}$.

4. Estudiar la adsorción disociativa de $\mathrm{N}_{2} \mathrm{O}$ en los cúmulos cargados y sin carga $\mathrm{Pt}_{8}$, $\mathrm{Pt}_{8}{ }^{ \pm}, \mathrm{Pt}_{8} \mathrm{O}, \mathrm{Pt}_{8} \mathrm{O}^{ \pm}$soportados en la superficie $\mathrm{TiO}_{2}$. Para determinar el efecto del soporte y de la reactividad de las nanoparticulas frente a la reduccion de la molecula de $\mathrm{N}_{2} \mathrm{O}$. 


\section{Bibliografía}

1. Te Velde, G., Bickelhaupt, F. M., van Gisbergen, S. J. A., Fonseca Guerra, C., Baerends, E. J., Snijders, J. G. and Ziegler, T. J. Comput. Chem. 22, 931 (2001). "Chemistry with ADF".

2. P. Perdew, K. Burke, and M. Ernzerhof. Physical Review Letters, 77, 3865 (1996). "Generalized Gradient Approximation Made Simple".

3. E. van Lenthe, E. J. Baerends, J. G, Snijders. J. Chem. Phys. 101 N $^{\circ}$ 11, 9783 (1994). "Relativistic total energy using regular approximations".

4. E. van Lenthe, J. G, Snijders, and E. J. Baerends. J. Chem. Phys. $105 \mathrm{~N}^{\circ}$ 15, 6505 (1996). "The zero-order regular approximation for relativistic effects: The effect of spin-orbit coupling in closed shell molecules".

5. E. van Lenthe, J. G, Snijders, and E. J. Baerends, J.G, Snijders. Int. J. Quantum Chem. 57, 281 (1996). "Relativistic regular two-components hamiltonians".

6. E. van Lenthe, R. van Leeuwen, E. J. Baerends. J. Chem. Phys. 110, 8943 (1999). "Geometry optimizations in the zero order regular approximation for relativistic effects".

7. “Nitrous Oxide and Climate Change”. Keith Smith (Editor) 2010. Pag. 1-3.

8. "Past and present in DeNO $O_{X}$ catalysis: from molecular modeling to chemical engineering”. Serie: Studies in Surface Science and Catalysis, volume 171. Pascal Granger (Editor), Vasile Pârvulescu (Editor) Editorial Elsevier, 2007. P. Granger, J.P: Dacquin, F. Dhainaut, C. Dujardin. Pages 291-324. Chapter 10. “The formation of $\mathrm{N}_{2} \mathrm{O}$ during $\mathrm{NO}_{X}$ conversion: fundamental approach and practical developments".

9. A. V. Leont'ev, O. A. Fomicheva, M. V. Proskurnina, N. S. Zefirov. Russian Chemical Revews 70(2), 91 (2001). "Modern chemistry of nitrous oxide”.

10. "Atmospheric Composition, Global Cyclies and Lifetimes

Orion".orion.it.luc.edu/ mschmel/Handout3.pdf 
11. «Kyoto Protocol: Status of Ratification». Organización de las Naciones Unidas. Convención Marco de las Naciones Unidas sobre el Cambio Climático (14 de enero de 2009).

12. "Programa de Naciones Unidas para el medio ambiente. Manual del Protocolo de Montreal relativo a las sustancias que agotan la capa de ozono". El sitio http://ozone.unep.org contiene información histórica y ajustes que se han ido haciendo desde 1990 a la actualidad.

13. F. Kapteijn, J. Rodríguez-Mirasol, J. A. Moulijin. Appl. Catal. B: Environmental 9, 25 (1996). "Heterogeneous catalytic decomposition of nitrous oxide”.

14. R. Burch, S.T. Daniells, J.P. Breen, P.Hu. Catal. Lett. 94, Nos 1-2, 103 (2004). "The effect of $\mathrm{H}_{2}$ and the presence of hot-O (ads) during the decomposition of $\mathrm{N}_{2} \mathrm{O}$ on platinum".

15. R. Burch, S.T. Daniells, J.P. Breen, P.Hu. J. Catal. 224, 252 (2004). “A combined transient and computational study of the dissociation of $\mathrm{N}_{2} \mathrm{O}$ on platinum catalysts".

16. D. B. Kang. Bull. Korean Chem. Soc. 28, № 12, 2369 (2007). "Theoretical study of the interaction of $\mathrm{N}_{2} \mathrm{O}$ with $\mathrm{Pd}(110)$.

17. H. Yamamoto, K. Miyajima, T. Yasuike, and F. Mafuné. J. Phys. Chem. A 117 (47), 12175 (2013). "Reactions of Neutral Platinum Clusters with $\mathrm{N}_{2} \mathrm{O}$ and CO".

18. D. P. Chen, K. Gai, Ch. Kong, Y. X, Han, L.-J. Hou, B.-W. Wu. J. At. Mol. Sci. 5, $\mathrm{N}^{\circ} 1,9$ (2014). "Density functional theory study of the catalytic reaction of $\mathrm{N}_{2} \mathrm{O}\left({ }^{1} \Sigma\right)$ with $\mathrm{CO}\left({ }^{1} \Sigma^{+}\right)$by $\mathrm{Ni}^{+}$".

19. F. Gonçalves, G. E. Marnellos, E.A. Efthimiadis, J.L. Figueiredo. React. Kinet. Lett. 80, $\mathrm{N}^{\circ}$ 153-159 (2003). "Simultaneous $\mathrm{N}_{2} \mathrm{O}$ and $\mathrm{NO}$ reduction over carbón supported catalysts".

20. Y. Izumi, T. Kizaki, K. Aika. Bull. Chem. Soc. Jpn. 74, 1499 (2001). "Simultaneous removal of Winter $\mathrm{NO}$ and $\mathrm{N}_{2} \mathrm{O}$ over Pd-ZSM-5 catalysts and HT-IR observations of their decomposition routes to $\mathrm{N}_{2}$ ”.

21. P. Khongpracha, Ch. Warakulwit, S. Namuangruk, M. Probst, J. Limtrakul. Chem Phys Chem 13, issue 2, 583 (2012). "Structures, energetics and reaction mechanisms of nitrous oxide on transition-metal-dope". 
22. E. R. S. Winter, J. Catal. 15 (1969) 144. "The decomposition of nitrous oxide on the rare-earth sesquioxides and related oxides".

23. E. R. S. Winter, J. Catal. 19, 32 (1970). "The decomposition of nitrous oxide on metallic oxides Part II."

24. E. R. S. Winter, J. Catal. 34, issue 3, 431 (1974). "The decomposition of $\mathrm{N}_{2} \mathrm{O}$ on oxide catalysts: III. The effect of $\mathrm{O}_{2}$ ".

25. J. Kaspar, P. Fornasiero and N. Hickey, Catal. Today, 77 (2003) 419. "Automotive catalytic converters: current status and some perspectives".

26. B. E. Nieuwenhuys. Advances Catal. 44, 259 2000. "The surface science approach toward understanding automotive exhaust conversion catalysis at the atomic level".

27. M. H. Kim, J. R. Ebner, R. M. Friedman, M. A. Vannice. Journal of Catalysis 204, 2 (2001) 348-357. "Disociative $\mathrm{N}_{2} \mathrm{O}$ Adsorption on Supported Pt".

28. D. H. Kim, M. H. Kim. Proceed. Korean Environmental Sci. Conference19, OA1) (2010). "Low-temperature $\mathrm{N}_{2} \mathrm{O}$ reduction by $\mathrm{H}_{2}$ with supported Pt nanoparticles".

29. A. C. Hermes, S. M. Hamilton, W. S. Hopkins, D.J. Harding, C. Kerpal, G. Meijer, A. Fielicke, S. R. Mackenzie. J. Phys. Chem. Letters 2 (2011) 3053-3057. "Effects of coadsorbed oxygen on the infrared driven decomposition of $\mathrm{N}_{2} \mathrm{O}$ on isolated $\mathrm{Rh}_{5}{ }^{+}$ clusters".

30. "Tables of Interatomic Distances and Configuration in Molecules and Ions (Nitrogen compounds)”, L. E. Sutton, ed., London: The Chemical Society, 1958.

31. S. K. Gupta, B. M. Nappi, K. A. Gingerich. Inorg. Chem. 20, issue 4, 966 (1981). "Mass Spectrometric Study of the Stabilities of the Gaseous Molecules Pt $t_{2}$ and PtY'.

32. K. Jansson, R. Scullman. J. Mol. Spectr. 61, 299-312 (1976) “Optical Absorption Spectra of PtO and $\mathrm{Pt}_{2}$ in Rare-Gas Matrices".

33. S. Taylor, G. W. Lemire, Y. M. Hamrick, Z. Fu, M.D. Morse. J. Chem. Phys. 89, 5517 (1988). "Resonant two-photon ionization spectroscopy of jet-cooled Pt $t_{2}$ ".

34. K. M. Ervin, J. Ho, W.C. Lineberger. J. Chem. Phys. 89, 5517 (1988). “Resonant two-photon ionization spectroscopy of jet-cooled $\mathrm{Pt}_{2}$ ".

35. I. Balint, A. Miyasaki. Dekker Encyclopedia of Nanoscience and Nanotechnology, Second Edition. 2009. 
36. J. Ho, M. L. Polak, K.M. Ervin, W. C. Lineberger. J. Chem. Phys. 99, 8542 (1993). "Photon electron spectroscopy of nickel group dimers: $\mathrm{Ni}_{2}, \mathrm{Pt}_{2}$, andPt $t_{2}$ ".

37. M. B. Airola and Michael D. Morse. J. Chem. Phys. 116, issue 4, 1313 (1993). "Rotationally resolved spectroscopy of $\mathrm{Pt}_{2}$ ". Michael D. Morse. Chem. Rev. 86, 1049 (1986). “Clusters of transition-Metal atoms".

38. K. Balasubramanian, J. Chem. Phys,.87 (1987) 6573. "Electronic states of Pt $t_{2}$ ".

39. Sang H. Yang, David A. Drabold, James B. Adams, Pablo Ordejón and Keith Glassford. J. Phys.: Condenced Matter 9 (5), L39 (1997). “Density functional studies of small platinum clusters.

40. L. Xiao, L. Wang. J. Phys. Chem. A 108 (issue 41), 8605 (2004). "Structures of Platinum Clusters: Planar or Spherical?"

41. A. Sachdev, R.I. Masel, J.B. Adams. Catal. Lett. 15, 57 (1992). "An investigation of small platinum clusters using the embedded atom method".

42. A. Nie, J. Wu, Ch. Zhou, S. Yao, Ch. Luo, R.C. Forrey, H. Cheng. Int. J. Quantum Chem. 107, issue 1, 219 (2007). "Structural evolution of subnano platinum clusters".

43. M. N. Huda, M. K. Niranjan, B.R. Sahu, L. Kleinman. Phys. Rev. A73, 053201, issue 5 (2006). "Effect of spin-orbit coupling on small platinum nanoclusters".

44. R. Avilés, E. Poulain, O. Olvera Neria, V. Bertin. J. Mol. Catal. A: Chem. 376, 22 (2013): "The spin significance in the capture and activation of $\mathrm{N}_{2} \mathrm{O}$ by small $\mathrm{Rh}$ nanopartcles".

45. E. K. Plyler, E. F. Barker. Phys. Rev. 38 (1931)1827. "The infrared spectrum and the molecular configuration of $\mathrm{N}_{2} 0$ ".

46. R. D. Nelson Jr., D. R. Lide, A. A. Maryott. "Selected Values of electric dipole moments for molecules in the gas phase". NSRDS-NBS10, 1967

47. R. Guirado-López, F. Aguilera-Granja, Phys. Lett. A 265, 116 (2000). “Nonmetalmetal transition in $R h_{N}$ and $R u_{N}$ clusters".

48. P. Bloński, S. Dennier, J. Hafner. J. Chem. Phys. 134, 034107, issue 3 (2011). "Strong spin-orbit effects in small Pt clusters: Geometric structure, magnetic isomers and anisotropy". 
49. K. Bhattacharyya, Ch. Majumder. Chem. Phys. Lett. 446, 4-6, 374 (2007). “Growth pattern and bonding trends in $\mathrm{Pt}_{n}(n=2-13)$ clusters: Theoretical investigation based on first principle calculations".

50. V. Kumar and Y. Kawazoe. Phys. Rev. B 77, issue 20, 205418 (2008). "Evolution of atomic and electronic structure of Pt clusters: Planar, layered, pyramidal, cage, cubic, and octahedral growth".

51. X. Wang, D. Tian. Comp. Mater. Sci. 46, 239 (2009). "Structures and structural evolution of $\mathrm{Pt}_{n}(n=15-24)$ clusters with combined density functional and genetic algorithm methods".

52. C. L. Heredia, V. Ferraresi-Curotto, M. B. López. Congreso SAM/CONAMET 2009. Buenos Aires 19/23/10/. "Estudio teórico de agregados de $\mathrm{Pt}_{\mathrm{n}}(\mathrm{n}=2-8)$ ”.

53. C. L. Heredia, V. Ferraresi-Curotto, M.B. López. Comp. Mater. Sci. 53, 18 (2012). "Characterization of $\mathrm{Pt}_{n}(n=2-12)$ clusters through global reactivity descriptors and vibrational spectroscopy, a theoretical study”.

54. A. Sebetci, Z.B. Güvenç. Surf. Sci. 525, 66 (2003). "Energetics and structures of small clusters: $P t_{N}, N=2-21$ ”.

55. A. Sebetci. Phys. Chem. Chem. Phys. 11, 921 (2009). "Does spin-orbit coupling effect favor planar structures for small platinum clusters?".

56. A. Sebetci. Comp. Materials Sci. 78, 9 (2013). "New minima for the Pt $t_{8}$ cluster".

57. I. Balteanu. P. Balaj. M. K. Beyer. Phys. Chem. Chem. Phys. 6, 2910 (2004). "Reactions of platinum clusters ${ }^{195} \mathrm{Pt}^{ \pm}, n=1-24$, with $\mathrm{N}_{2} \mathrm{O}$ studied with isotopically enriched platinum".

58. www.scm.com/Products/Overview/frozencore.html. 24 de octubre del 2014.

59. Ernest R. Davidson and Subhas Chakravorty. Theor Chim Acta 83, 319-330 (1992). "A test of the Hirshfeld definition of atomic charges and moments".

60. F. L. Hirshfeld. Theoret. Claim. Acta (Berl.) 44, 129-138 (1977). "Bonded-Atom Fragments for Describing Molecular Charge Densities". 


\section{Anexos}

\subsection{ADF : Ámsterdam Density Functional Software}

El programa Amsterdan Density Fuctional (ADF) es un programa de cálculo basado en primeros principios de estructura electrónica que hace uso de la teoría de funcionales de la densidad (DTF). Desarrollado en primer lugar en los años setentas por el grupo de E. J. Baerends de la Universidad de Vrije en Ámsterdam, y por el grupo de T. Ziegler de la Universidad de Calgary.

Es usado por investigadores, académicos y la industria, en muchos campos como farmacoquímica y ciencia de materiales. Este último en particularmente popular en aéreas de investigación tales como en la catálisis homogénea y heterogénea, química inorgánica, elementos químicos pesados, varios tipos de espectroscopia y bioquímica.

La teoría detrás del paquete ADF la aproximación de Kohn-Sham a la Teoría de Funcionales de la Densidad. Este es un importante método computacional de primeros principios de estructura electrónica para predecir y aproximar las propiedades físicas y químicas y analizarlas e interpretar de manera conveniente en términos químicos sencillos.

Algunas características y capacidades especificas en este programa:

1. Orbitales Tipo Slater (STOs) como funciones de base, en contraste con otros códigos que usan orbitales Tipo Gausianas (GTOs).

2. Conjunto de bases y métodos relativistas (aproximación de orden cero a la ecuación de Dirac (ZORA) y acoplamiento espín-orbita) para todos los elementos de la tabla periódica hasta el elemento 118.

3. Varias propiedades moleculares: IR, Ramman, VCD, UV, XAS espectros; parámetros NMR Y EPR (ESR).

4. Efectos de solventes y ambientes vía COSMOS, COSMO-RS, QM/MM; DRF. 
5. Interfaz grafica (GUI) de ADF y BAND hace fácil hacer el cálculo y visualizar los resultados.

Como un poderoso paquete de cálculo de la DFT, este programa tiene diversas funcionalidades, de las cuales se podrán utilizar para realizar las siguientes tareas:

i. Geometría de optimización: encuentra la geometría optimizada para la molécula deseada.

ii. Cálculos de estado de transición: encuentra el estado de transición o barrera de activación entre reactivos y productos y observa la estructura correspondiente a ese estado de transición.

iii. Frecuencias de vibración verifica que la estructura global optimizada no tenga frecuencias imaginarias y de transición que tenga exactamente un valor de frecuencia de vibración imaginaria y propiedades termodinámicas (entropía, energía interna y capacidad calorífica a volumen constante de la molécula).

\subsubsection{Aproximación de core congelado}

El programa ADF emplea la aproximación de core congelado para reducir el tiempo de computo mediante la reducción del tamaño del conjunto de base variacional. En el núcleo los orbitales atómicos más cercanos a este no cambian mucho respecto a la descripción de formación de enlaces, la aproximación de núcleo congelado ahorra tiempo sin sacrificar mucho la calidad de los resultados. Con un cálculo de núcleo congelado se obtiene la densidad de carga total, el potencial de la valencia y en la región del núcleo, haciendo caso omiso de sólo el ligero cambio en los orbitales más cercanos al núcleo en la formación del enlace químico. 
Para garantizar la ortogonalidad entre los orbitales básicos congelados y las funciones de base de valencia, el conjunto base de valencia se utiliza en las ecuaciones de SCF (Self Consistent Field) se ortogonalizan explícitamente al núcleo congelado.

Esto se logra mediante la adición a la valencia establecida una serie de funciones básicas auxiliares $\chi_{\mu}{ }^{\text {core }}$, una para cada orbital de núcleo congelado. Cada función de valencia se sustituye por una combinación lineal

$$
X_{v}^{\text {valencia }} \rightarrow X_{v}^{\text {valencia }}+\sum_{\mu} c_{\mu v} X_{\mu}^{\text {core }} \quad \text { Ec.7 }
$$

La condición de que cada una de tales funciones de valencia modificada sea ortogonal a cada orbital de núcleo congelado determinando exactamente todos los coeficientes $\boldsymbol{c}_{\boldsymbol{\mu} \boldsymbol{v}}$. Los orbitales básicos congelados se han tomado de cálculos precisos de un solo átomo con conjuntos de base grandes STO (Slater Type Orbitals).

El programa ADF ofrece para la mayoría de los átomos en la tabla periódica varios conjuntos de base con diferentes "niveles" de aproximación de núcleo congelado, incluyendo conjuntos de base para todos los electrones para evitar la aproximación de núcleo congelado por completo [58].

\subsubsection{Efectos Relativistas}

Los metales de transición representan frecuentemente la fase activa de los catalizadores. El estudio teórico de los catalizadores pasa necesariamente por describir de manera satisfactoria a los metales de transición, lo cual implica considerar los efectos relativistas a los que se ven sujetos los electrones internos de los átomos pesados.

La ecuación de Dirac incluye la relatividad en su formulación, pero resulta demasiado compleja para sistemas más allá de unos cuantos átomos, por lo que se han propuesto varias alternativas. Una de ellas es el formalismo de Pauli en donde se resuelve de manera 
aproximada la ecuación de Dirac a través de la reducción de la función de onda de cuatro a dos componentes, sin embargo, la solución de Pauli presenta problemas de convergencia.

Otra de las alternativas para resolver de manera aproximada la ecuación de Dirac es el esquema computacional de dos componentes que es variacionalmente estable en campos Coulómbicos (en átomos y moléculas) conocido como la aproximación regular de orden cero (ZORA, Zero Order Regular Approximation) [5, 3 y 6 ].

Este esquema puede ser considerado como una expansión perturbativa a la ecuación de Dirac, donde ZORA representa el orden más bajo. El método ZORA incluye explícitamente la relatividad, la única diferencia entre un cálculo convencional en la aproximación de Kohn-Sham y un cálculo ZORA es el término de energía cinética:

(a)

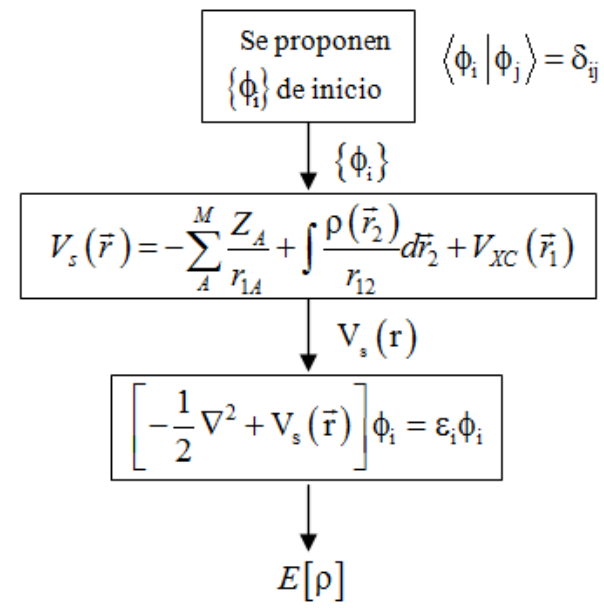

(b)

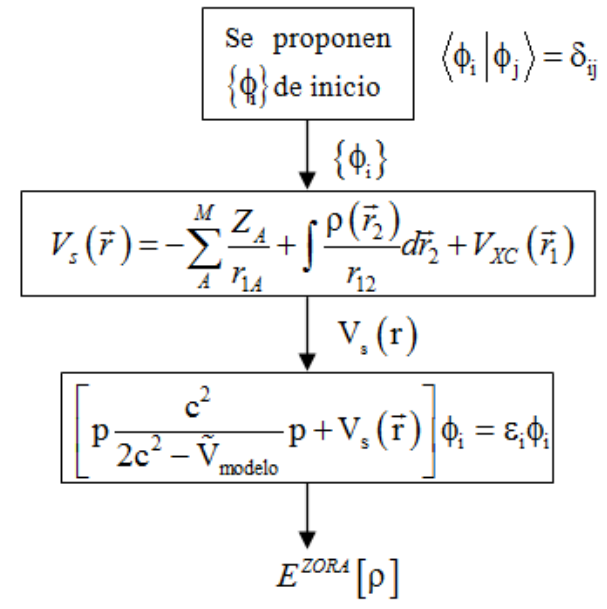

Esquema 1. (a) Aproximación de Khon-Sham para cálculos autoconsistentes, donde la energía depende de la densidad electrónica. (b) En la aproximación de ZORA, el operador de energía cinética $-\frac{1}{2} \nabla^{2}$ se reemplaza por $\mathrm{p} \frac{\mathrm{c}^{2}}{2 \mathrm{c}^{2}-\tilde{\mathrm{V}}_{\text {modelo }}} \mathrm{p}$.

El potencial modelo está definido como

$$
\tilde{V}_{\text {modelo }}=-\sum_{A} \frac{Z_{A}}{\left|\vec{r}-\vec{R}_{A}\right|}+\int \frac{\rho_{\text {modelo }}\left(\vec{r}^{\prime}\right)}{\left|\vec{r}-\vec{r}^{\prime}\right|} d r^{\prime}+V_{X C}^{L D A}\left[\rho_{\text {modelo }}\right] \quad E c .8
$$


que depende de una densidad modelo construida a partir de la suma de las densidades atómicas $\rho_{\text {model }}(\vec{r})=\sum_{A} \rho^{A}(\vec{r})$, con $\rho^{A}(\vec{r})$ obtenida a través de una combinación lineal de

funciones gaussianas $\rho^{A}(\vec{r})=\sum_{i} d_{i} \exp \left(-\alpha_{i}\left|\vec{r}-\vec{R}_{A}\right|^{2}\right) \cdot V_{X C}^{L D A}$ representa el potencial de intercambio-correlación para la aproximación de densidad local (LDA, local density approximation). La aproximación ZORA permite tratar explícitamente la relatividad. De tal manera, con la introducción de este formalismo en ADF, el método ZORA es una aproximación recomendable para cálculos relativistas con ADF.

\subsubsection{Ecuacion de Dirac}

La ecuación de Dirac (Ecuación 3) da una descripción de las partículas elementales de espín 1/2, como el electrón. Esta ecuación fue originalmente formulada para describir el electrón pero actualmente se aplica a otros tipos de partículas elementales de espín 1/2, como los quarks, está escrita de la siguiente manera:

$$
\left(\alpha_{0} m c^{2}+\sum_{j=1}^{3} \alpha_{j} p_{j} c\right) \psi(x, t)=i \hbar \frac{\partial \psi}{\partial t}(x, t) \quad E c .9
$$

donde $\mathrm{H}$ es el Hamiltoniano de Dirac

$$
H=\left(\alpha_{0} m c^{2}+\sum_{j=1}^{3} \alpha_{j} p_{j} c\right)
$$

siendo $m$ la masa en reposo del electrón, $c$ la velocidad de la luz, $p$ el operador de momento, $\hbar$ la constante reducida de Planck, $\mathbf{x}$ y $t$ las coordenadas del espacio y el tiempo, respectivamente; у $\psi(\mathbf{x}, t)$ una función de onda de cuatro componentes (formulada como un espinor, similar a un vector que cambia de signo con una rotación de $2 \pi$ descubierto por Pauli y Dirac), de cuatro componentes, $\alpha$ son operadores lineales que gobiernan la función 
de onda, escritos como una matriz y son matrices de $4 \times 4$ conocidas como matrices de Dirac.

La ecuación de Dirac describe las amplitudes de probabilidad para un electrón solo. Esta teoría de una sola partícula da una predicción suficientemente buena del espín y del momento magnético del electrón, y explica la mayor parte de la estructura fina observada en las líneas espectrales atómicas.

\subsection{2 .2 Formalismo de Pauli}

En el formalismo de Pauli los efectos relativistas se incluyen en el primer orden con $1 / c^{2}$, donde $c$ es la velocidad de la luz. El Hamiltoniano de Pauli tiene términos relativistas dados por la siguiente ecuación:

$$
H_{\text {Pauli }}=-\frac{p^{4}}{8 m^{3} c^{2}}+\frac{\Delta V}{8 m c^{2}}+\frac{1}{4 m c^{2}} \sigma \cdot(\nabla V \times p) \quad \text { Ec.11 }
$$

donde $\boldsymbol{p}$ es el operador de momento, $\boldsymbol{m}$ la masa, $\boldsymbol{c}$ la velocidad de la luz y $\boldsymbol{V}$ es el potencial de Coulomb $(\boldsymbol{V}=-\boldsymbol{Z} / \boldsymbol{r})$. También definido de la siguiente manera:

$$
H_{\text {Pauli }} \equiv H_{M V}+H_{D}+H_{S O}
$$

El termino $\boldsymbol{H}_{\boldsymbol{M} V}$ describe el incremento relativista de la masa del electrón con la velocidad, y puede considerarse una corrección a la energía cinética no relativista. El término de Darwin $\boldsymbol{H}_{\boldsymbol{D}}$ es la contribución no clásica del llamado Zitterbewegungn, i.e., El efecto mecánico-cuántico asociado con la rapidez del movimiento de los electrones alrededor de sus posiciones medias. $\boldsymbol{H}_{M V}$ y $\boldsymbol{H}_{D}$ son los términos escalares relativistas. 
El termino spin-orbita $\boldsymbol{H}_{S o}$ resultado de la interacción del momento magnético del electrón con el campo magnético generado por su propio movimiento orbital.

\subsubsection{Cargas atómicas}

En ADF existen tres diferentes aproximaciones para determinar las cargas atómicas: (1) el método de deformación de la densidad de Voronoi (VDD), (2) el esquema Hirshfeld y finalmente (3) el análisis de población de Mulliken. De estos tres solo los dos primeros son usados con frecuencias ya que el último tiene fuerte dependencia con la base empleada.

\subsubsection{Método de deformación de la densidad de Voronoi (VDD)}

El primer método (VDD) está basado en la deformación de la densidad y una rigurosa partición del espacio dentro de áreas atómicas no superpuestas, llamada celda de Voronoi. La celda de Voronoi de un átomo A es una región en un espacio cerrado del núcleo A y se puede determinar de la siguiente manera:

$$
\begin{aligned}
Q_{A}^{V D D} & =-\int_{\substack{\text { ceronoi } \\
\text { vell }}}\left(\rho(r)-\sum_{B} \rho_{B}(r)\right) d r \\
& =-\int_{\substack{\text { Voronoi } \\
\text { cell } A}} \Delta \rho(r)_{\text {def }} d r
\end{aligned}
$$

El método de VDD se resume en la deformación de la densidad en tres dimensiones $\Delta \rho(r)_{d e f}$ sobre una base por átomo. 


\subsubsection{Análisis de población de Hirshfeld}

El análisis de población de Hirshfeld, es un método que está basado directamente en la densidad electrónica, hace uso de ésta en la molécula y en una promolécula ficticia. La promolécula es definida como la suma sobre las densidades atómicas del estado basal (usualmente promediadas esféricamente). En la promolécula la densidad total es una suma de contribuciones bien definidas de todos los átomos constituyentes:

$$
\rho_{\text {promolécula }}(\vec{r})=\Sigma_{B} \rho_{B}(\vec{r})
$$

Todo lo que tenemos que hacer es imitar esta composición atómica de la densidad promolécula en el reparto de la densidad molecular real entre los átomos. De acuerdo con ello, se divide la densidad molecular en cada punto entre los átomos de la molécula en proporción a sus respectivas contribuciones a la densidad promolecular en ese punto [5960]. A partir de esta densidad se encuentra la densidad de cada fragmento o átomo, A, a partir de:

$$
\rho_{A}^{\text {promolécula }}(r)=\frac{\rho_{A}(r)}{\rho_{\text {promolécula }}(r)} \rho^{\text {molécula }}(r) d r \quad \text { Ec. } 15
$$

La carga de Hirsfeld es obtenida al restar la integral de la densidad asociada con el átomo A de la correspondiente carga nuclear, $\mathrm{Z}_{\mathrm{A}}$ :

$$
Q_{A}=Z_{A}-\int \frac{\rho_{A}(r)}{\rho_{\text {promolécula }}(r)} \rho^{\text {molécula }}(r) d r \quad \text { Ec. } 16
$$

De la ecuación anterior, se ve que el método de Hirshfeld toma en cuenta de manera natural el hecho de que cada átomo tiene un cierto tamaño característico. En este trabajo se empleó el enfoque de Hirshfeld para determinar las cargas de cada uno de los átomos, por ser de los métodos confiables y dar mejores resultados, ya que este no depende de la base empleada. 


\subsubsection{Teoría de Funcionales de la Densidad.}

La teoría de funcionales de la densidad (DFT) representa una alternativa a los métodos tradicionales basados en la función de onda. Aunque es un método aproximado, es capaz de describir el intercambio y la correlación de manera más fácil y menos costosa que los métodos pos-HF. La base de esta teoría radica en la idea de que la energía del estado fundamental de un sistema polielectrónico depende solamente de la función de densidad electrónica, $\boldsymbol{\rho}(\boldsymbol{r})$, de manera que no es necesario conocer la función de onda que describa al sistema. La función de densidad electrónica viene a suponer la probabilidad de encontrar un electrón en un volumen con espín arbitrario.

Los orígenes de esta teoría se encuentran en un modelo desarrollado por Thomas y Fermi a finales del año1920. Pero hasta mediados de los años 1960 con las contribuciones de Hohenber, Kohn y Sham se establecieron el formalismo teórico en el que se basa esta teoría.

\subsubsection{Teoremas de Hohenberg y Kohn}

El primer teorema de Hohenberg y Kohn, establece que la densidad electrónica del estado fundamental, $\rho_{0}(\boldsymbol{r})$, no puede derivar de dos potenciales distintos, salvo que ambos difieran por una constante. En particular, Hohenberg y Kohn mostraron que la energía es un funcional de la densidad a través de la relación

$$
E[\rho]=F[\rho]+\int d \mathbf{r} \rho(\mathbf{r}) v(\mathbf{r}) \quad E c .17
$$

Donde $F[\rho]$ representa al funcional universal que contiene a la energía cinética, $T[\rho]$, y la interacción electrón-electrón, $V_{e e}[\rho]$. 
Con un segundo teorema los mismos autores demostraron que la densidad electrónica del estado basal es aquella que minimiza al funcional de energía $E[\rho]$ y por lo tanto define el correcto potencial externo.

$$
E_{0} \leq E[\rho]
$$

Desde el punto de vista numérico, la función de onda es un objeto muy complejo de manipular, pues para $N$ partículas es una función de $3 N$ variables, mientras que la densidad es más fácil de manejar pues es siempre una función de 3 variables, independientemente del número de partículas. El problema, es que no se conoce la forma exacta del funcional $F[\rho]$.

\subsubsection{Modelo de Kohn-Sham}

Kohn y Sham presentaron una forma de aproximar al funcional universal. Estos autores señalaron que un sistema polielectrónico descrito por una densidad podría ser sustituido por otro sistema de electrones no interactuantes con la misma densidad. Esto significa que tal sistema puede estar representado por un determinante (determinante de Slater) cuyos elementos son funciones que representan a cada uno de los electrones del sistema (orbitales, $\phi_{i}$ ). Con este punto de partida la energía cinética corresponde a una suma de energías cinéticas individuales

$$
T_{s}=\sum_{i=1}^{N} \int d \mathbf{r} \phi_{i}^{*}(\mathbf{r})\left(-\frac{1}{2} \nabla^{2}\right) \phi_{i}(\mathbf{r})
$$

y la densidad electrónica a la suma de densidades orbitales

$$
\rho(\mathbf{r})=\sum_{i=1}^{N} \rho_{i}(\mathbf{r}) \sum_{i=1}^{N} \phi_{i}^{*}(\mathbf{r}) \phi_{i}(\mathbf{r}) \quad \text { Ec. } 19
$$


Un elemento adicional en el modelo de Kohn y Sham es la aproximación a la interacción electrón-electrón ya que proponen como parte principal de ésta a la interacción coulómbica:

$$
J[\rho]=\frac{1}{2} \iint d \mathbf{r} d \mathbf{r}^{\prime} \frac{\rho(\mathbf{r}) \rho\left(\mathbf{r}^{\prime}\right)}{\left|\mathbf{r}-\mathbf{r}^{\prime}\right|}
$$

y con esto el funcional universal es escrito como:

$$
F[\rho]=T[\rho]+V_{e e}[\rho]=T_{s}[\rho]+J[\rho]+E_{x c}[\rho]
$$

donde el funcional de intercambio y correlación es:

$$
E_{x c}[\rho]=T[\rho]-T_{s}[\rho]+V_{e e}[\rho]-J[\rho]
$$

Los orbitales de Kohn y Sham son aquellos que satisfagan las ecuaciones integrodiferenciales de Kohn y Sham

$$
\left(-\frac{1}{2} \nabla^{2}+v_{\mathrm{eff}}(\mathbf{r})\right) \phi_{i}(\mathbf{r})=\varepsilon_{i} \phi_{i}(\mathbf{r})
$$

y que generarán la energía del estado basal. El potencial de Kohn-Sham $v_{\text {eff }}(\mathbf{r})$ incluye las interacciones coulombianas (electrón- electrón y núcleo-electrón) y de intercambio y correlación. El único problema que se presenta en esta teoría, es que no se conoce la expresión universal del funcional de correlación e intercambio.

\subsubsection{Intercambio y correlación}

Aún cuando el planteamiento de Kohn y Sham es exacto, hasta el momento el funcional de intercambio y correlación exacto, $E_{x c}$, es desconocido y por lo tanto son necesarias aproximaciones a este funcional. La clasificación a estas aproximaciones se puede encontrar en la escalera de Jacob definida por John. P. Perdew. 
La primera aproximación conocida como Aproximación de Densidad Local (LDA) es el primer eslabón a la escalera de Jacob y la manera más sencilla posible para calcular la energía de correlación e intercambio. Consiste en suponer que en cada punto, la energía de intercambio y correlación depende sólo de la densidad en ese punto. Este valor se considera como el que tendría un gas de electrones libres de esa densidad. Se obtienen resultados sorprendentemente precisos para algunas propiedades.

El segundo peldaño de la escalera de Jacob es la Aproximación de Gradiente Generalizado (GGA), estas son semilocales, ya que consideran en cada punto el valor de la densidad y sus gradientes. Un ejemplo de esta aproximación es el funcional reportado por Perdew, Burke y Ernzerhof. Para algunas propiedades estas aproximaciones dan mejores resultados que LDA, en particular para geometrías moleculares y energías del estado fundamental, aunque para otras no representan una mejora sustancial.

Aún con esta aproximación, no se conoce la forma funcional para las energías de correlación electrónicas y energía de intercambio. Estas corresponden a la interacción cuántica entre electrones, la primera debido a la parte cuántica de la repulsión coulombiana y la segunda debido al principio de exclusión de Pauli entre electrones del mismo espín. Una solución a este problema es el desarrollo de métodos híbridos como el B3LYP, pero existen otros métodos dentro de la propia teoría del funcional de la densidad.

Una serie de funcionales más sofisticados puede obtenerse al suponer que la energía de intercambio y correlación depende explícitamente de los orbitales de Kohn-Sham. El más común de estos funcionales es el de Intercambio Exacto (EXX), que incluye de manera completa la energía de intercambio electrónico y que puede derivarse desde primeros principios. El problema de este tipo de funcionales es que computacionalmente son más costosos de tratar. 


\subsubsection{Continuación resultados $\mathrm{N}_{2} \mathrm{O}+\mathrm{Pt}_{8}$}

I. Interacción en átomos de Pt.

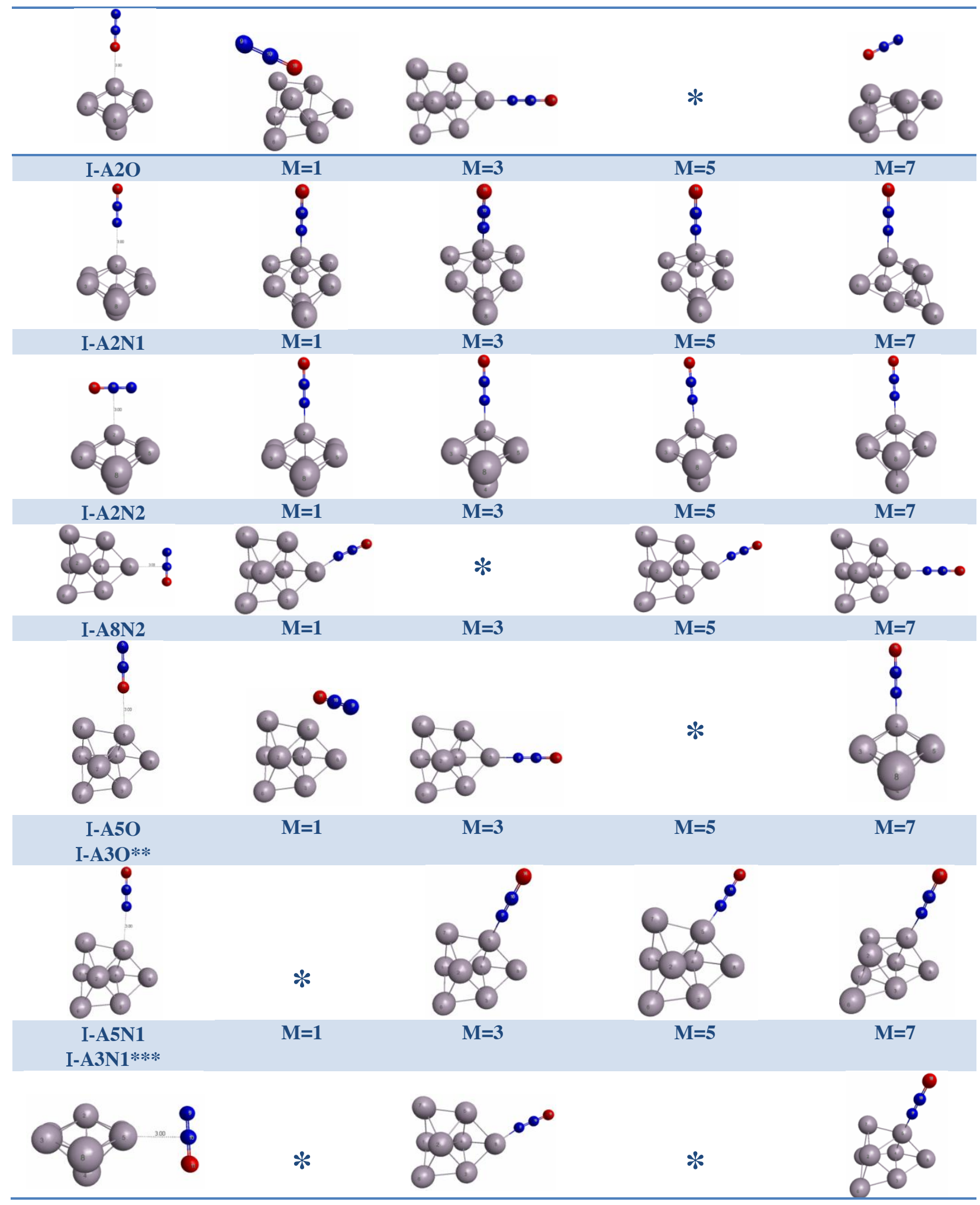




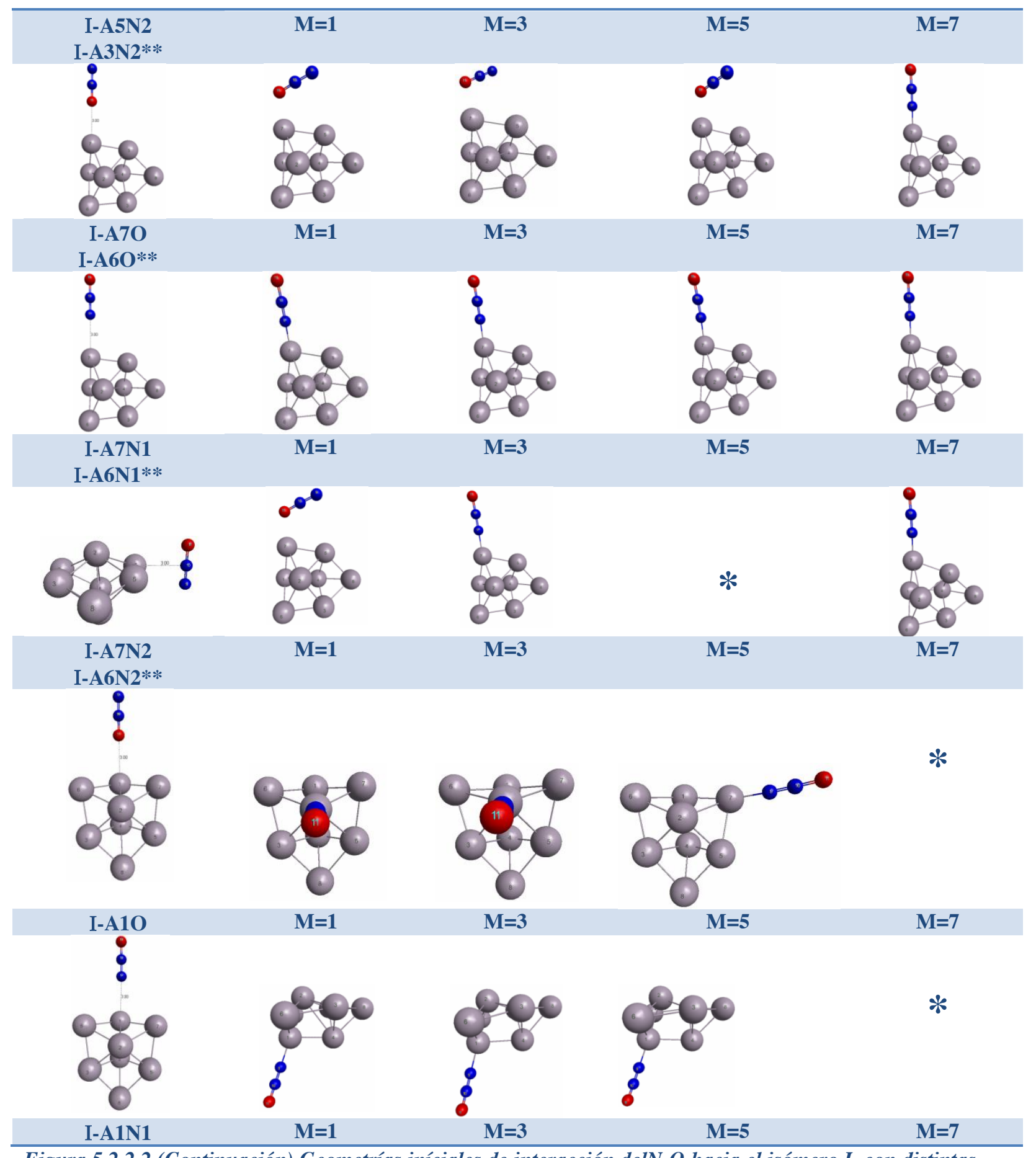

Figura 5.2.2 2.2 (Continuación) Geometrías iníciales de interacción del $\mathrm{N}_{2} \mathrm{O}$ hacia el isómero I, con distintas multiplicidades de espín $\left.(M=2 s+1) .{ }^{*}\right)$ Problemas de convergencia en el cálculo, no se obtienen resultados.(**) interacciones en sitios equivalentes al indicado, por simetría del isómero I. 
Tabla 5.2.2.1 (Continuación) Parámetros energéticos, interacción, $\mathrm{Pt}_{8}+\mathrm{N}_{2} \mathrm{O}$, por átomos de la nanopartícula de $P_{8}$. Energías de disociación $\left(E_{\text {dis }}\right)$ y energía relativa $\left(E_{\text {relativa }}\right)$.

\begin{tabular}{|c|c|c|c|c|}
\hline $\begin{array}{c}\text { Tipo de } \\
\text { interacción }\end{array}$ & $\begin{array}{c}M \\
2 s+1\end{array}$ & $\begin{array}{c}\mathbf{E}_{\text {dis }} \\
\mathrm{kcal} / \mathrm{mol}\end{array}$ & $\begin{array}{c}\mathrm{E}_{\text {relativa }} \\
\mathrm{kcal} / \mathrm{mol}\end{array}$ & $\begin{array}{c}\text { Enlace } \\
\text { /disociación }\end{array}$ \\
\hline \multirow{4}{*}{$\mathrm{I}-\mathrm{A} 2 \mathrm{O}$} & $\bar{~} 1$ & 1.99 & 64.50 & $\mathrm{Si} / \mathrm{no}$ \\
\hline & 3 & -14.72 & 47.79 & $\mathrm{Si} /$ no \\
\hline & 5 & --- & --- & $*$ \\
\hline & 7 & 3.22 & 65.74 & $\mathrm{Si} / \mathrm{no}$ \\
\hline \multirow{4}{*}{$\mathrm{I}-\mathrm{A} 2 \mathrm{~N} 1$} & 1 & -11.96 & 50.55 & $\mathrm{Si} / \mathrm{no}$ \\
\hline & 3 & -17.87 & 44.64 & $\mathrm{Si} / \mathrm{no}$ \\
\hline & 5 & -13.96 & 48.55 & $\mathrm{Si} /$ no \\
\hline & 7 & -16.57 & 45.94 & $\mathrm{Si} /$ no \\
\hline \multirow{4}{*}{$\mathrm{I}-\mathrm{A} 2 \mathrm{~N} 2$} & 1 & -11.97 & 50.55 & $\mathrm{Si} / \mathrm{no}$ \\
\hline & 3 & -17.85 & 44.66 & $\mathrm{Si} /$ no \\
\hline & 5 & -13.95 & 48.56 & $\mathrm{Si} /$ no \\
\hline & 7 & -11.12 & 51.39 & $\mathrm{Si} / \mathrm{no}$ \\
\hline \multirow{4}{*}{ I-A8N2 } & 1 & -9.81 & 52.71 & $\mathrm{Si} / \mathrm{no}$ \\
\hline & 3 & --- & --- & $*$ \\
\hline & 5 & -12.24 & 50.28 & $\mathrm{Si} /$ no \\
\hline & 7 & -10.86 & 51.66 & $\mathrm{Si} /$ no \\
\hline \multirow{4}{*}{$\mathrm{I}-\mathrm{A} 5 \mathrm{O}$} & 1 & 8.19 & 70.70 & $\mathrm{Si} / \mathrm{no}$ \\
\hline & 3 & -14.74 & 47.78 & $\mathrm{Si} /$ no \\
\hline & 5 & -13.97 & 48.54 & $\mathrm{Si} /$ no \\
\hline & 7 & --- & --- & $*$ \\
\hline \multirow{4}{*}{ I-A 5N1 } & 1 & --- & --- & $*$ \\
\hline & 3 & -19.09 & 43.43 & $\mathrm{Si} /$ no \\
\hline & 5 & -10.97 & 51.54 & $\mathrm{Si} /$ no \\
\hline & 7 & -12.68 & 49.84 & $\mathrm{Si} /$ no \\
\hline \multirow{4}{*}{$\mathrm{I}-\mathrm{A} 5 \mathrm{~N} 2$} & 1 & --- & --- & $*$ \\
\hline & 3 & -19.09 & 43.43 & $\mathrm{Si} / \mathrm{no}$ \\
\hline & 5 & --- & --- & $*$ \\
\hline & 7 & -13.35 & 49.17 & $\mathrm{Si} /$ no \\
\hline \multirow{4}{*}{$\mathrm{I}-\mathrm{A} 7 \mathrm{O}$} & 1 & -0.39 & 62.12 & $\mathrm{Si} / \mathrm{no}$ \\
\hline & 3 & -2.31 & 60.20 & $\mathrm{Si} /$ no \\
\hline & 5 & -3.18 & 59.33 & $\mathrm{Si} /$ no \\
\hline & 7 & --- & --- & $*$ \\
\hline \multirow{4}{*}{ I-A7N1 } & 1 & -17.12 & 45.39 & $\mathrm{Si} / \mathrm{no}$ \\
\hline & 3 & -19.15 & 43.36 & $\mathrm{Si} /$ no \\
\hline & 5 & -19.05 & 43.46 & $\mathrm{Si} /$ no \\
\hline & 7 & -14.28 & 48.23 & $\mathrm{Si} /$ no \\
\hline
\end{tabular}




\begin{tabular}{ccccc}
\hline & 1 & -0.39 & 62.13 & $\mathrm{Si} / \mathrm{no}$ \\
I-A7N2 & 3 & -19.07 & 43.44 & $\mathrm{Si} / \mathrm{no}$ \\
& 5 & --- & --- & $*$ \\
& 7 & -14.27 & 48.24 & $\mathrm{Si} / \mathrm{no}$ \\
\hline \multirow{2}{*}{ I-A1O } & 1 & -11.96 & 50.55 & $\mathrm{Si} / \mathrm{no}$ \\
& 3 & -17.85 & 44.66 & $\mathrm{Si} / \mathrm{no}$ \\
& 5 & -19.05 & 43.47 & $\mathrm{Si} / \mathrm{no}$ \\
$\mathrm{I}-\mathrm{A} 1 \mathrm{~N} 1$ & 7 & --- & --- & $*$ \\
& 1 & -18.13 & 44.38 & $\mathrm{Si} / \mathrm{no}$ \\
& 3 & -22.94 & 39.57 & $\mathrm{Si} / \mathrm{no}$ \\
& 5 & -19.44 & 43.07 & $\mathrm{Si} / \mathrm{no}$ \\
& 7 & --- & & $*$ \\
\hline \hline
\end{tabular}

*Problemas de convergencia no hay resultados. 
Tabla 5.2.2.2 (Continuación) Parámetros geométricos, interacción, $\mathrm{Pt}_{8}+\mathrm{N}_{2} \mathrm{O}$, por átomos de la nanopartícula de Pt. Multiplicidad (M), Frecuencias de vibración (v) y distancias de enlace (d) .

\begin{tabular}{|c|c|c|c|c|c|c|}
\hline $\begin{array}{c}\text { Tipo de } \\
\text { interacción }\end{array}$ & $\begin{array}{c}M \\
2 s+1\end{array}$ & $\begin{array}{c}v^{N-N} \mathbf{c m}^{-} \\
1\end{array}$ & $\begin{array}{c}\mathbf{d}_{\text {Pt-O }} \\
\AA\end{array}$ & $\begin{array}{c}\mathbf{d P t}_{\mathbf{P}} \\
\AA\end{array}$ & $\begin{array}{c}\mathbf{d}_{\mathbf{P t}-\mathbf{P t}} \\
\AA\end{array}$ & $\begin{array}{c} \pm \mathbb{đ}_{\mathbf{P t}-P t} \\
\AA\end{array}$ \\
\hline \multirow{4}{*}{$\mathrm{I}-\mathrm{A} 2 \mathrm{O}$} & $\bar{~} 1$ & 2263 & 2.75 & - & 2.58 & 0.05 \\
\hline & 3 & 1243 & - & 1.98 & 2.60 & 0.08 \\
\hline & 5 & --- & --- & --- & $*$ & $*$ \\
\hline & 7 & 2268 & 2.67 & - & 2.60 & 0.1 \\
\hline \multirow{4}{*}{ I-A2N1 } & 1 & 1243 & - & 1.97 & 2.58 & 0.05 \\
\hline & 3 & 1258 & - & 1.97 & 2.59 & 0.08 \\
\hline & 5 & 1252 & - & 1.94 & 2.59 & 0.08 \\
\hline & 7 & 1250 & - & 1.93 & 2.64 & 0.12 \\
\hline \multirow{4}{*}{ I-A2N2 } & 1 & 1244 & - & 1.97 & 2.59 & 0.08 \\
\hline & 3 & 1257 & - & 1.97 & 2.59 & 0.08 \\
\hline & 5 & 1251 & - & 1.94 & 2.60 & 010 \\
\hline & 7 & 1250 & - & 1.94 & 2.60 & 0.10 \\
\hline \multirow{4}{*}{ I-A8N2 } & 1 & 1246 & - & 1.96 & 2.61 & 0.11 \\
\hline & 3 & --- & --- & --- & --- & $*$ \\
\hline & 5 & 1239 & - & 1.98 & 2.60 & 0.11 \\
\hline & 7 & 1254 & - & 1.94 & 2.60 & 0.11 \\
\hline \multirow{4}{*}{$\mathrm{I}-\mathrm{A} 5 \mathrm{O}$} & 1 & 2259 & 2.42 & - & 2.61 & 0.11 \\
\hline & 3 & 1244 & - & 1.98 & 2.62 & 0.10 \\
\hline & 5 & 1253 & - & 1.94 & 2.60 & 0.10 \\
\hline & 7 & --- & --- & --- & --- & $*$ \\
\hline \multirow{4}{*}{ I-A5N1 } & 1 & --- & --- & --- & --- & $*$ \\
\hline & 3 & 1254 & - & 1.96 & 2.59 & 0.08 \\
\hline & 5 & 1250 & - & 2.01 & 2.59 & 0.06 \\
\hline & 7 & 1231 & - & 2.01 & 2.67 & 0.06 \\
\hline \multirow{4}{*}{$\mathrm{I}-\mathrm{A} 5 \mathrm{~N} 2$} & 1 & --- & --- & --- & --- & $*$ \\
\hline & 3 & 1254 & - & 1.96 & 2.59 & 0.08 \\
\hline & 5 & --- & --- & --- & --- & $*$ \\
\hline & 7 & 1253 & - & 1.95 & 2.60 & 0.10 \\
\hline \multirow{4}{*}{$\mathrm{I}-\mathrm{A} 7 \mathrm{O}$} & 1 & 2242 & 2.34 & - & 2.59 & 0.08 \\
\hline & 3 & 2258 & 2.49 & - & 2.61 & 0.11 \\
\hline & 5 & 2255 & 2.34 & - & 2.59 & 0.08 \\
\hline & $7 *$ & --- & --- & --- & --- & $*$ \\
\hline \multirow{3}{*}{ I-A7N1 } & 1 & 1251 & - & 1.93 & 2.62 & 0.1 \\
\hline & 3 & 1254 & - & 1.93 & 2.59 & 0.08 \\
\hline & 5 & 1253 & - & 1.94 & 2.59 & 0.08 \\
\hline
\end{tabular}




\begin{tabular}{ccccccc}
\hline & 7 & 1253 & - & 1.94 & 2.6 & 0.1 \\
\hline \multirow{3}{*}{ I-A7N2 } & 1 & 2243 & 2.34 & - & 2.5 & 0.08 \\
& 3 & 1254 & - & 1.93 & 2.59 & 0.08 \\
& 5 & --- & --- & --- & --- & $*$ \\
& 7 & 1252 & - & 1.94 & 2.59 & 0.08 \\
\hline \multirow{2}{*}{ I-A1O } & 1 & 1244 & - & 1.97 & 2.59 & 0.08 \\
& 3 & 1258 & - & 1.97 & 2.59 & 0.08 \\
& 5 & 1253 & - & 1.94 & 2.59 & 0.08 \\
& 7 & --- & --- & --- & --- & $*$ \\
\hline & 1 & 1257 & - & 1.95 & 2.61 & 0.11 \\
& 3 & 1255 & - & 1.95 & 2.58 & 0.05 \\
& 5 & 1253 & - & 1.94 & 2.59 & 0.08 \\
& 7 & --- & --- & --- & --- & $*$ \\
\hline \hline
\end{tabular}

*Problemas de convergencia no hay resultados. 
I. Interacción en enlaces de Pt.

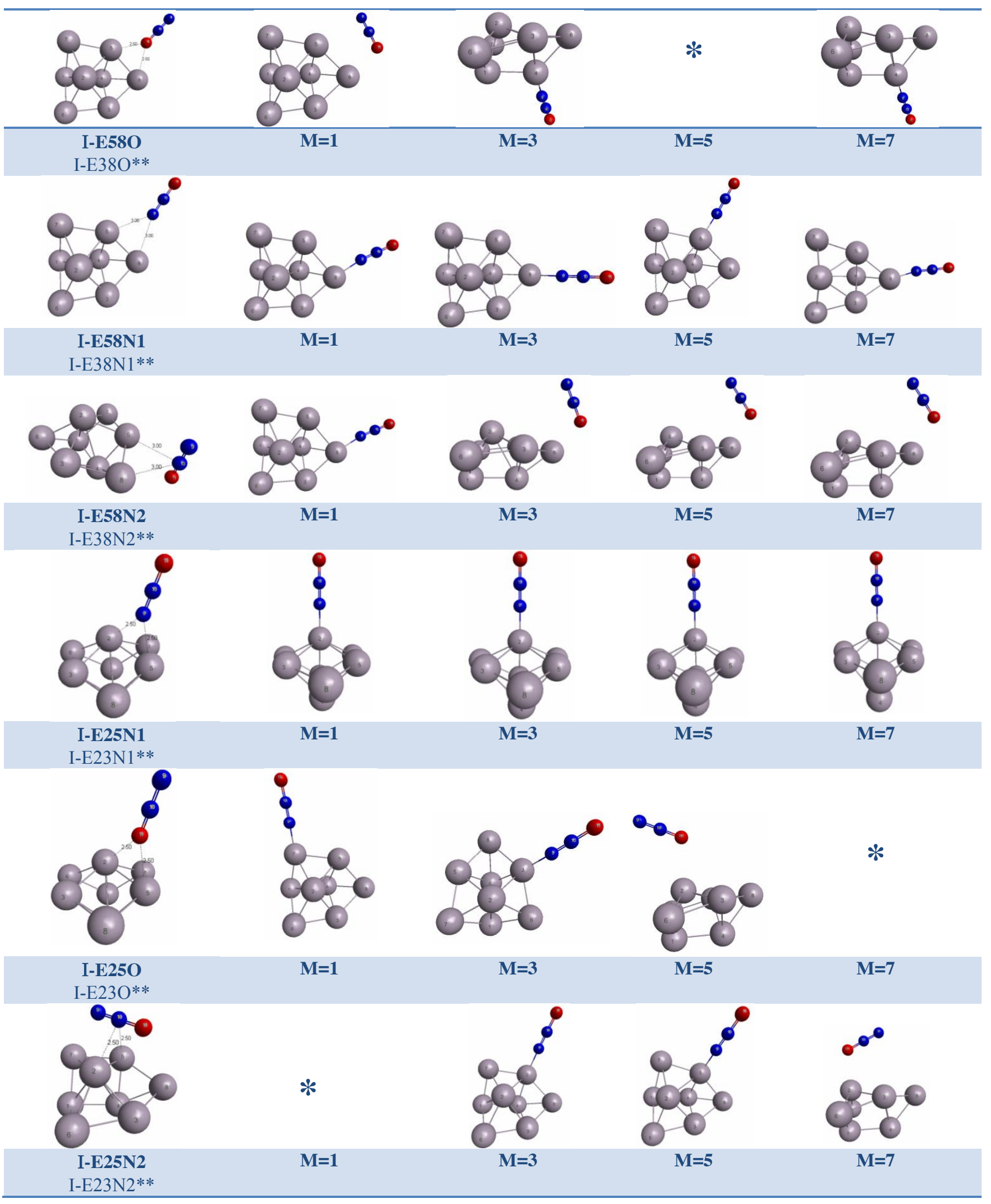




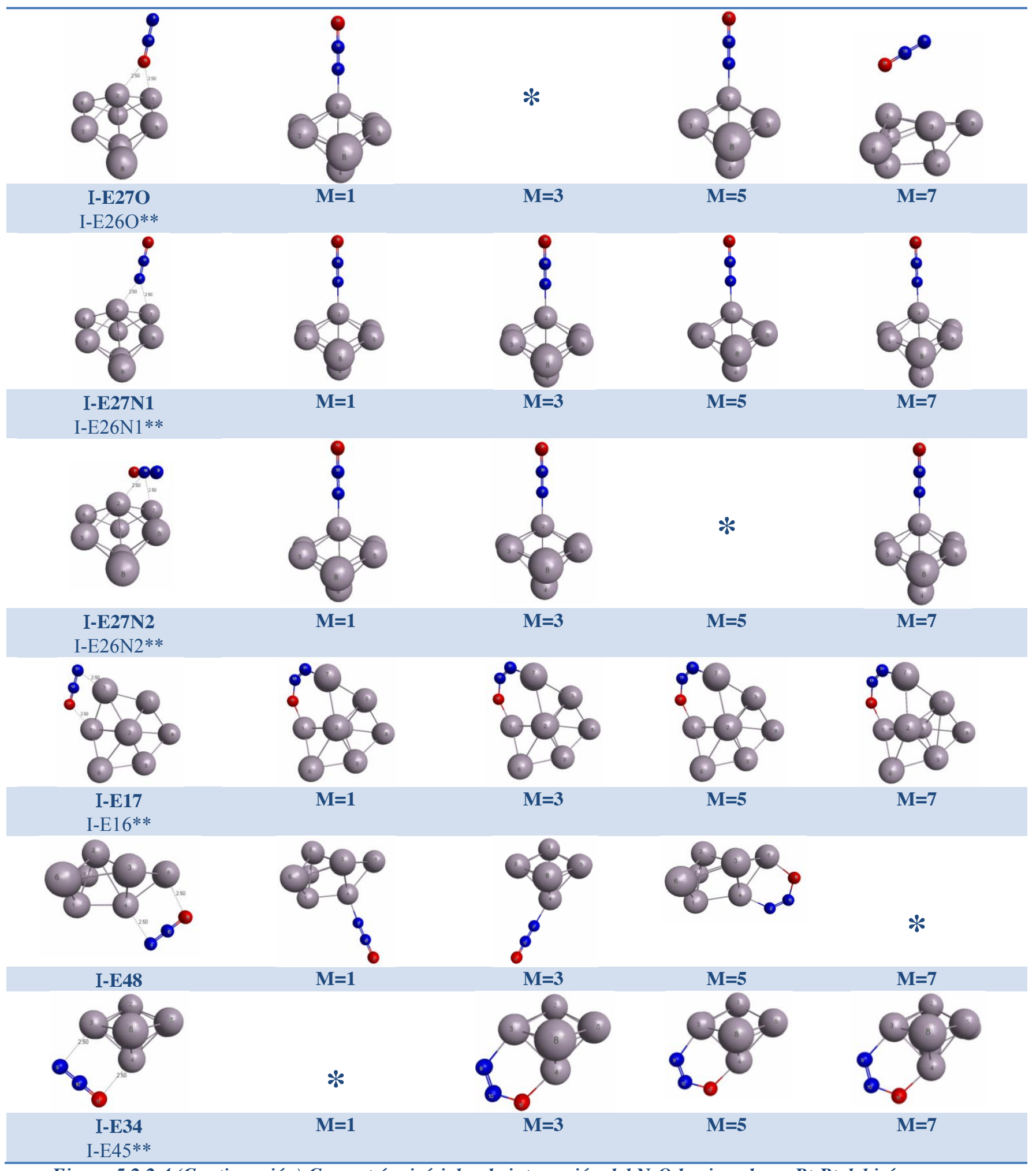

Figura 5.2.2.4 (Continuación) Geometrías iníciales de interacción del $\mathrm{N}_{2} \mathrm{O}$ hacia enlaces Pt-Pt del isómero $I$, con distintas multiplicidades de espín $(M=2 s+1)$. (*) Problemas de convergencia en el cálculo, no se obtienen resultados.(**) interacciones en sitios equivalentes al indicado, por simetría del isómero $I$. 
Tabla 5.2.2.3 (Continuación) Parámetros de energía, interacción $\mathrm{Pt}_{8}+\mathrm{N}_{2} \mathrm{O}$ por enlaces. Energías de disociación $\left(E_{\text {dis }}\right)$, energía relativa $\left(E_{\text {relativa }}\right)$.

\begin{tabular}{|c|c|c|c|c|}
\hline $\begin{array}{c}\text { Tipo de } \\
\text { interacción }\end{array}$ & $\begin{array}{c}M \\
2 s+1\end{array}$ & $\begin{array}{c}\mathbf{E}_{\mathrm{dis}} \\
\mathrm{kcal} / \mathrm{mol}\end{array}$ & $\begin{array}{c}\text { E }_{\text {relativa }} \\
\text { kcal/mol }\end{array}$ & $\begin{array}{c}\text { Enlace / } \\
\text { disociación }\end{array}$ \\
\hline \multirow{4}{*}{ I-E58O } & 1 & 1.61 & 70.27 & $\mathrm{Si} / \mathrm{no}$ \\
\hline & 3 & -19.24 & 49.43 & $\mathrm{Si} /$ no \\
\hline & 5 & --- & --- & $*$ \\
\hline & 7 & -14.95 & 53.72 & $\mathrm{Si} / \mathrm{no}$ \\
\hline \multirow{4}{*}{ I-E58N1 } & 1 & -9.80 & 58.87 & $\mathrm{Si} /$ no \\
\hline & 3 & -14.74 & 53.93 & $\mathrm{Si} /$ no \\
\hline & 7 & -7.40 & 57.70 & $\mathrm{Si} / \mathrm{no}$ \\
\hline & 5 & -10.97 & 61.27 & $\mathrm{Si} /$ no \\
\hline \multirow{4}{*}{ I-E58N2 } & 1 & -9.81 & 58.86 & $\mathrm{Si} / \mathrm{no}$ \\
\hline & 3 & -2.95 & 65.71 & $\mathrm{Si} /$ no \\
\hline & 5 & -1.92 & 66.75 & $\mathrm{Si} / \mathrm{no}$ \\
\hline & 7 & 2.40 & 71.07 & $\mathrm{Si} / \mathrm{no}$ \\
\hline \multirow{4}{*}{$\mathrm{I}-\mathrm{E} 25 \mathrm{~N} 1$} & 1 & -11.50 & 57.17 & $\mathrm{Si} / \mathrm{no}$ \\
\hline & 3 & -17.87 & 50.80 & $\mathrm{Si} / \mathrm{no}$ \\
\hline & 5 & -14.06 & 54.61 & $\mathrm{Si} /$ no \\
\hline & 7 & -11.12 & 57.55 & $\mathrm{Si} /$ no \\
\hline \multirow{4}{*}{ I-E25O } & 1 & -17.12 & 51.55 & $\mathrm{Si} / \mathrm{no}$ \\
\hline & 3 & -19.11 & 49.56 & $\mathrm{Si} / \mathrm{no}$ \\
\hline & 5 & -0.45 & 68.22 & $\mathrm{Si} /$ no \\
\hline & 7 & --- & --- & $*$ \\
\hline \multirow{4}{*}{ I-E25N2 } & 1 & --- & --- & --- \\
\hline & 3 & -19.11 & 49.56 & $\mathrm{Si} / \mathrm{no}$ \\
\hline & 5 & -18.26 & 50.41 & $\mathrm{Si} /$ no \\
\hline & 7 & 3.24 & 71.91 & $\mathrm{Si} / \mathrm{no}$ \\
\hline \multirow{4}{*}{ I-E27O } & 1 & -11.96 & 56.71 & $\mathrm{Si} / \mathrm{no}$ \\
\hline & 3 & --- & --- & $*$ \\
\hline & 5 & -13.95 & 54.72 & $\mathrm{Si} / \mathrm{no}$ \\
\hline & 7 & 3.23 & 71.90 & $\mathrm{Si} / \mathrm{no}$ \\
\hline \multirow{4}{*}{ I-E27N1 } & 1 & -11.96 & 56.71 & $\mathrm{Si} / \mathrm{no}$ \\
\hline & 3 & -17.84 & 50.83 & $\mathrm{Si} / \mathrm{no}$ \\
\hline & 5 & -13.97 & 54.70 & $\mathrm{Si} / \mathrm{no}$ \\
\hline & 7 & -11.12 & 57.55 & $\mathrm{Si} / \mathrm{no}$ \\
\hline \multirow{4}{*}{ I-E27N2 } & 1 & -11.96 & 56.71 & $\mathrm{Si} /$ no \\
\hline & 3 & -17.85 & 50.82 & $\mathrm{Si} /$ no \\
\hline & 5 & --- & --- & $*$ \\
\hline & 7 & -11.12 & 57.55 & $\mathrm{Si} / \mathrm{no}$ \\
\hline
\end{tabular}




\begin{tabular}{ccccc}
\hline \multirow{2}{*}{ I-E17N2 } & 1 & -15.35 & 53.32 & $\mathrm{Si} / \mathrm{no}$ \\
& 3 & -21.95 & 46.72 & $\mathrm{Si} / \mathrm{no}$ \\
& 5 & -19.34 & 49.33 & $\mathrm{Si} / \mathrm{no}$ \\
& 7 & -16.26 & 52.41 & $\mathrm{Si} / \mathrm{no}$ \\
\hline \multirow{3}{*}{$\mathrm{I}-\mathrm{E} 48$} & 1 & -14.48 & -54.19 & $\mathrm{SI} / \mathrm{no}$ \\
& 3 & -19.24 & 49.93 & $\mathrm{Si} / \mathrm{no}$ \\
& 5 & --- & --- & $*$ \\
& 7 & --- & --- & $*$ \\
\hline \multirow{2}{*}{$\mathrm{I}-\mathrm{E} 34$} & 1 & --- & --- & $\mathrm{Si} / \mathrm{no}$ \\
& 3 & -9.07 & 59.59 & $\mathrm{Si} / \mathrm{no}$ \\
& 5 & -9.90 & 58.77 & $\mathrm{Si} / \mathrm{no}$ \\
\hline \hline
\end{tabular}

*Problemas de convergencia no hay resultados. 
Tabla 5.2.2.4 (Continuación) Parámetros geométricos, interacción $\mathrm{Pt}_{8}+\mathrm{N}_{2} \mathrm{O}$ por enlaces. Multiplicidad (M), Frecuencias de vibración (v) y distancias de enlace $(d)$.

\begin{tabular}{|c|c|c|c|c|c|c|}
\hline $\begin{array}{c}\text { Tipo de } \\
\text { interacción }\end{array}$ & $\begin{array}{c}M \\
2 s+1\end{array}$ & $\begin{array}{l}v^{N-N} \\
\mathrm{~cm}^{-1}\end{array}$ & $\begin{array}{c}\mathbf{d}_{\mathbf{P t - O}} \\
\AA\end{array}$ & $\begin{array}{c}\mathbf{d}_{\text {Pt-N }} \\
\stackrel{\AA}{\mathbf{A}}\end{array}$ & $\begin{array}{c}\mathbf{d}_{\mathbf{P t}-\mathbf{P t}} \\
\AA\end{array}$ & 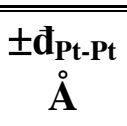 \\
\hline \multirow{4}{*}{ I-E58O } & $\overline{11}$ & 2263 & 2.66 & 4.07 & 2.59 & "0.08 \\
\hline & 3 & 1259 & - & 1.97 & 2.58 & 0.08 \\
\hline & 5 & --- & --- & --- & --- & $*$ \\
\hline & 7 & 1251 & - & 1.96 & 2.62 & 0.10 \\
\hline \multirow{4}{*}{ I-E58N1 } & 1 & 1248 & - & 1.96 & 2.62 & 0.10 \\
\hline & 3 & 1245 & - & 1.98 & 2.62 & 0.10 \\
\hline & 5 & 1250 & - & 2.01 & 2.62 & 0.08 \\
\hline & 7 & 1240 & - & 1.99 & 2.59 & 0.09 \\
\hline \multirow{4}{*}{ I-E58N2 } & 1 & 1248 & - & 1.96 & 2.62 & 0.10 \\
\hline & 3 & 2264 & 2.49 & - & 2.61 & 0.11 \\
\hline & 5 & 2264 & 2.64 & - & 2.58 & 0.08 \\
\hline & 7 & 2264 & 2.52 & - & 2.59 & 0.08 \\
\hline \multirow{4}{*}{ I-E25N1 } & 1 & 1243 & - & 1.97 & 2.59 & 0.08 \\
\hline & 3 & 1258 & - & 1.97 & 2.59 & 0.09 \\
\hline & 5 & 1255 & - & 1.97 & 2.60 & 0.10 \\
\hline & 7 & 1251 & - & 1.97 & 2.60 & 0.10 \\
\hline \multirow{4}{*}{ I-E25O } & 1 & 1251 & - & 1.93 & 2.62 & 0.10 \\
\hline & 3 & 1255 & - & 1.94 & 2.63 & 0.08 \\
\hline & 5 & 2270 & 2.88 & - & 2.59 & 0.08 \\
\hline & 7 & --- & --- & --- & --- & $*$ \\
\hline \multirow{4}{*}{ I-E25N2 } & 1 & --- & --- & --- & --- & $*$ \\
\hline & 3 & 1255 & - & 1.96 & 2.59 & 0.08 \\
\hline & 5 & 1253 & - & 1.95 & 2.60 & 0.10 \\
\hline & 7 & 2267 & 2.63 & - & 2.59 & 0.08 \\
\hline \multirow{4}{*}{ I-E27O } & 1 & 1243 & - & 1.95 & 2.59 & 0.08 \\
\hline & 3 & --- & --- & --- & --- & $*$ \\
\hline & 5 & 1253 & - & 1.94 & 2.60 & 0.10 \\
\hline & 7 & 1269 & 2.66 & - & 2.69 & 0.08 \\
\hline \multirow{4}{*}{ I-E27N1 } & 1 & 1243 & - & 1.97 & 2.59 & 0.08 \\
\hline & 3 & 1256 & - & 1.97 & 2.59 & 0.08 \\
\hline & 5 & 1252 & - & 1.95 & 2.60 & 0.10 \\
\hline & 7 & 1250 & - & 1.94 & 2.60 & 0.09 \\
\hline \multirow{4}{*}{ I-E27N2 } & 1 & 1243 & - & 1.97 & 2.59 & 0.08 \\
\hline & 3 & 1257 & - & 1.97 & 2.59 & 0.08 \\
\hline & 5 & --- & --- & --- & --- & $*$ \\
\hline & 7 & 1250 & - & 1.94 & 2.60 & 0.10 \\
\hline
\end{tabular}




\begin{tabular}{ccccccc}
\hline \multirow{2}{*}{ I-E17N2 } & 1 & 1544 & 2.03 & 1.91 & 2.59 & 0.08 \\
& 3 & 1528 & 2.03 & 1.91 & 2.60 & 0.10 \\
& 5 & 1526 & 2.00 & 1.91 & 2.60 & 0.10 \\
& 7 & 1541 & 2.02 & 1.91 & 2.61 & 0.11 \\
\hline \multirow{2}{*}{ I-E48 } & 1 & 1243 & - & 1.97 & 2.59 & 0.08 \\
& 3 & 1259 & - & 1.97 & 2.58 & 0.05 \\
& 5 & --- & --- & --- & --- & $*$ \\
& 7 & --- & --- & --- & --- & $*$ \\
\hline \multirow{2}{*}{ I-E34 } & 1 & --- & --- & --- & --- & $*$ \\
& 3 & 1573 & 2.08 & 2.02 & 2.59 & 0.05 \\
& 5 & 1578 & 2.08 & 2.01 & 2.59 & 0.05 \\
& 7 & 1489 & 2.03 & 2.00 & 2.60 & 0.06 \\
\hline \hline
\end{tabular}

*Problemas de convergencia no hay resultados. 
II. Interacción en caras de Pt.

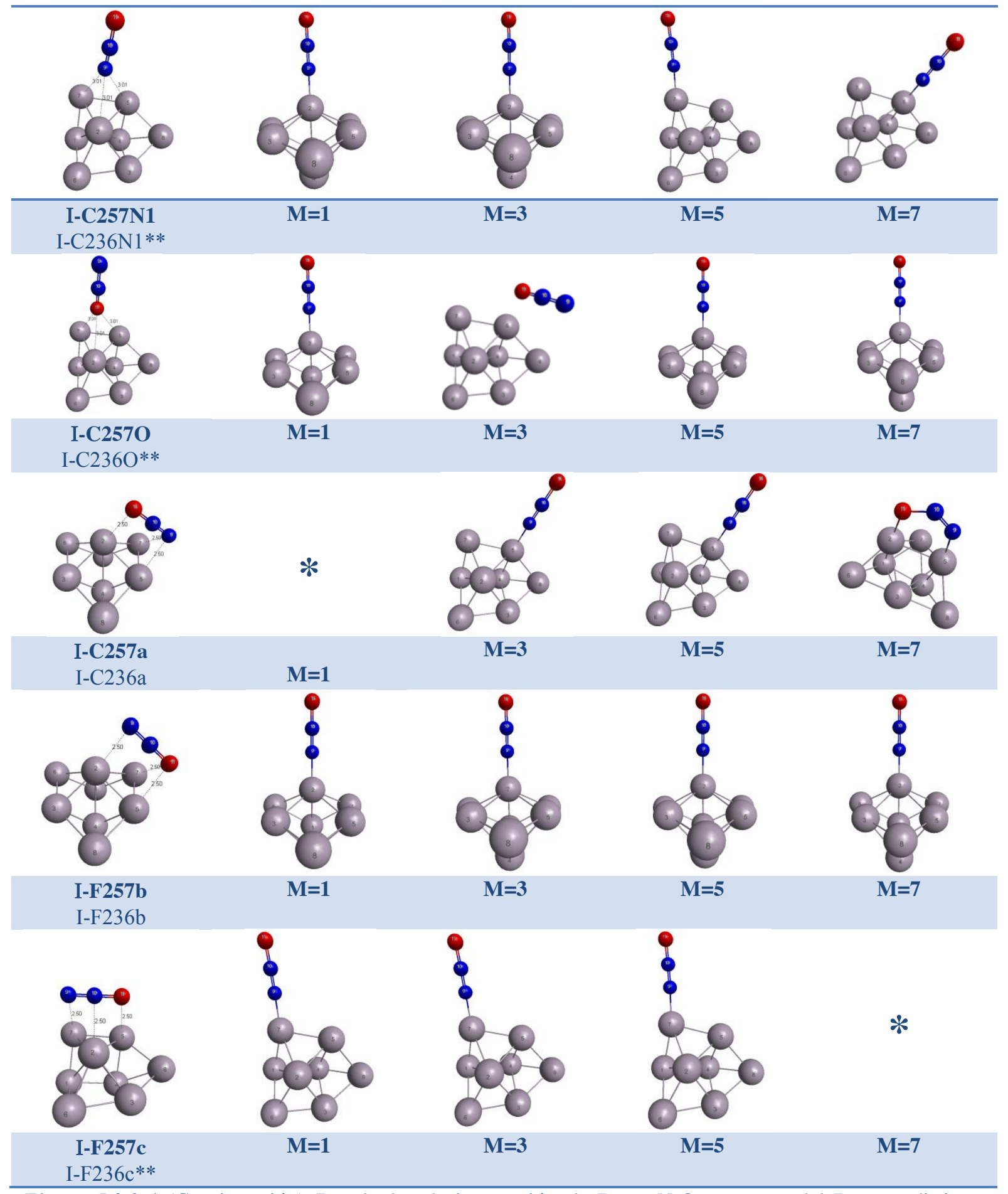

Figura 5.2.2.6 (Continuación) Resultados de interacción de $\mathrm{Pt}_{8}+\mathrm{N}_{2} \mathrm{O}$ en caras del Pt, con distintas multiplicidades de espín $(M=2 s+1)$. (*) Problemas de convergencia en el cálculo, no se obtienen resultados. $\left.{ }^{* *}\right)$ interacciones en sitios equivalentes al indicado, por simetría del isómero I. 
Tabla 5.2.2.5 (Continuación) Parámetros de energía, interacción $\mathrm{Pt}_{8}+\mathrm{N}_{2} \mathrm{O}$ por caras. Energías de disociación $\left(E_{\text {dis }}\right)$ y energía relativa $\left(E_{\text {relativa }}\right)$.

\begin{tabular}{ccccc}
\hline \hline $\begin{array}{c}\text { Tipo de } \\
\text { interacción }\end{array}$ & $\begin{array}{c}\mathbf{M} \\
\mathbf{2 s + 1}\end{array}$ & $\begin{array}{c}\mathbf{E}_{\mathbf{d i s}} \\
\mathbf{k c a l} / \mathbf{m o l}\end{array}$ & $\begin{array}{c}\mathbf{E}_{\text {relativa }} \\
\text { kcal/mol }\end{array}$ & $\begin{array}{c}\text { Enlace } \\
\text { /disociación }\end{array}$ \\
\hline \hline \multirow{2}{*}{ I-C257 } & 1 & -11.96 & 7.15 & $\mathrm{Si} /$ no \\
& 3 & -17.87 & 1.23 & $\mathrm{Si} /$ no \\
& 5 & -19.05 & 0.05 & $\mathrm{Si} /$ no \\
& 7 & -13.35 & 5.76 & $\mathrm{Si} /$ no \\
\hline \multirow{2}{*}{ I-C257O } & 1 & -11.96 & 7.14 & $\mathrm{Si} /$ no \\
& 5 & -13.98 & 15.53 & $\mathrm{Si} /$ no \\
& 7 & -11.13 & 5.12 & $\mathrm{Si} /$ no \\
& 3 & -3.57 & 7.98 & $\mathrm{Si} /$ no \\
\hline I-C257a & $1 *$ & --- & --- & $*$ \\
& 3 & -19.11 & 0.00 & $\mathrm{Si} /$ no \\
& 5 & -18.20 & 0.91 & $\mathrm{Si} /$ no \\
& 7 & -16.96 & 2.14 & $\mathrm{Si} /$ no \\
\hline I-C257b & 1 & -11.96 & 7.15 & $\mathrm{Si} /$ no \\
& 3 & -17.85 & 1.26 & $\mathrm{Si} /$ no \\
& 5 & -13.95 & 5.15 & $\mathrm{Si} /$ no \\
& 7 & -11.13 & 7.98 & $\mathrm{Si} /$ no \\
\hline \hline
\end{tabular}

*Problemas de convergencia no hay resultados. 
Tabla 5.2.2.6 (Continuación) Parámetros geométricos, interacción $\mathrm{Pt}_{8}+\mathrm{N}_{2} \mathrm{O}$ por enlaces. Multiplicidad (M), Frecuencias de vibración (v) y distancias de enlace $(d)$.

\begin{tabular}{|c|c|c|c|c|c|c|}
\hline $\begin{array}{c}\text { Tipo de } \\
\text { interacción }\end{array}$ & $\begin{array}{c}M \\
2 s+1\end{array}$ & $\begin{array}{l}v^{N-N} \\
\mathbf{c m}^{-1}\end{array}$ & $\underset{\AA}{\mathbf{d}_{\text {Pt-O }}}$ & $\underset{\AA}{\stackrel{\mathbf{d}_{\mathrm{Pt}-\mathrm{N}}}{\mathrm{A}}}$ & $\begin{array}{c}\mathbf{d}_{\mathbf{P t}-\mathrm{Pt}} \\
\AA\end{array}$ & $\underset{\AA}{\mathbf{\AA}}$ \\
\hline & $\bar{~} 1$ & 1243 & - & 1.97 & 2.58 & 0.05 \\
\hline \multirow[t]{4}{*}{$\mathrm{I}-\mathrm{C} 257 \mathrm{~N} 1$} & 3 & 1258 & - & 1.97 & 2.59 & 0.08 \\
\hline & 5 & 1254 & - & 1.94 & 2.59 & 0.08 \\
\hline & 7 & 1253 & - & 1.95 & 2.59 & 0.06 \\
\hline & 1 & 1244 & - & 1.97 & 2.58 & 0.05 \\
\hline \multirow[t]{4}{*}{$\mathrm{I}-\mathrm{C} 257 \mathrm{O}$} & 3 & 2259 & 2.38 & - & 2.62 & 0.10 \\
\hline & 5 & 1254 & - & 1.94 & 2.59 & 0.08 \\
\hline & 7 & 1251 & - & 1.94 & 2.6 & 0.10 \\
\hline & 1 & --- & --- & --- & --- & $*$ \\
\hline \multirow[t]{4}{*}{$\mathrm{I}-\mathrm{C} 257 \mathrm{a}$} & 3 & 1255 & - & 1.96 & 2.59 & 0.08 \\
\hline & 5 & 1252 & - & 1.95 & 2.59 & 0.08 \\
\hline & 7 & 1498 & 2.01 & 1.94 & 2.65 & 0.12 \\
\hline & 1 & 1243 & - & 1.97 & 2.58 & 0.05 \\
\hline \multirow[t]{4}{*}{$\mathrm{I}-\mathrm{C} 257 \mathrm{~b}$} & 3 & 1256 & - & 1.97 & 2.59 & 0.08 \\
\hline & 5 & 1253 & - & 1.94 & 2.60 & 0.10 \\
\hline & 7 & 1251 & - & 1.94 & 2.58 & 0.05 \\
\hline & 1 & 1250 & - & 1.93 & 2.62 & 0.10 \\
\hline \multirow[t]{3}{*}{$\mathrm{I}-\mathrm{C} 257 \mathrm{c}$} & 3 & 1254 & - & 1.93 & 2.59 & 0.08 \\
\hline & 5 & 1254 & - & 1.94 & 2.59 & 0.08 \\
\hline & 7 & --- & --- & --- & --- & $*$ \\
\hline
\end{tabular}




\section{1 .6 Continuación de resultados $\mathrm{N}_{2} \mathrm{O}+\mathrm{Pt}_{8} \mathrm{O}$}

I. Interaction en átomos.
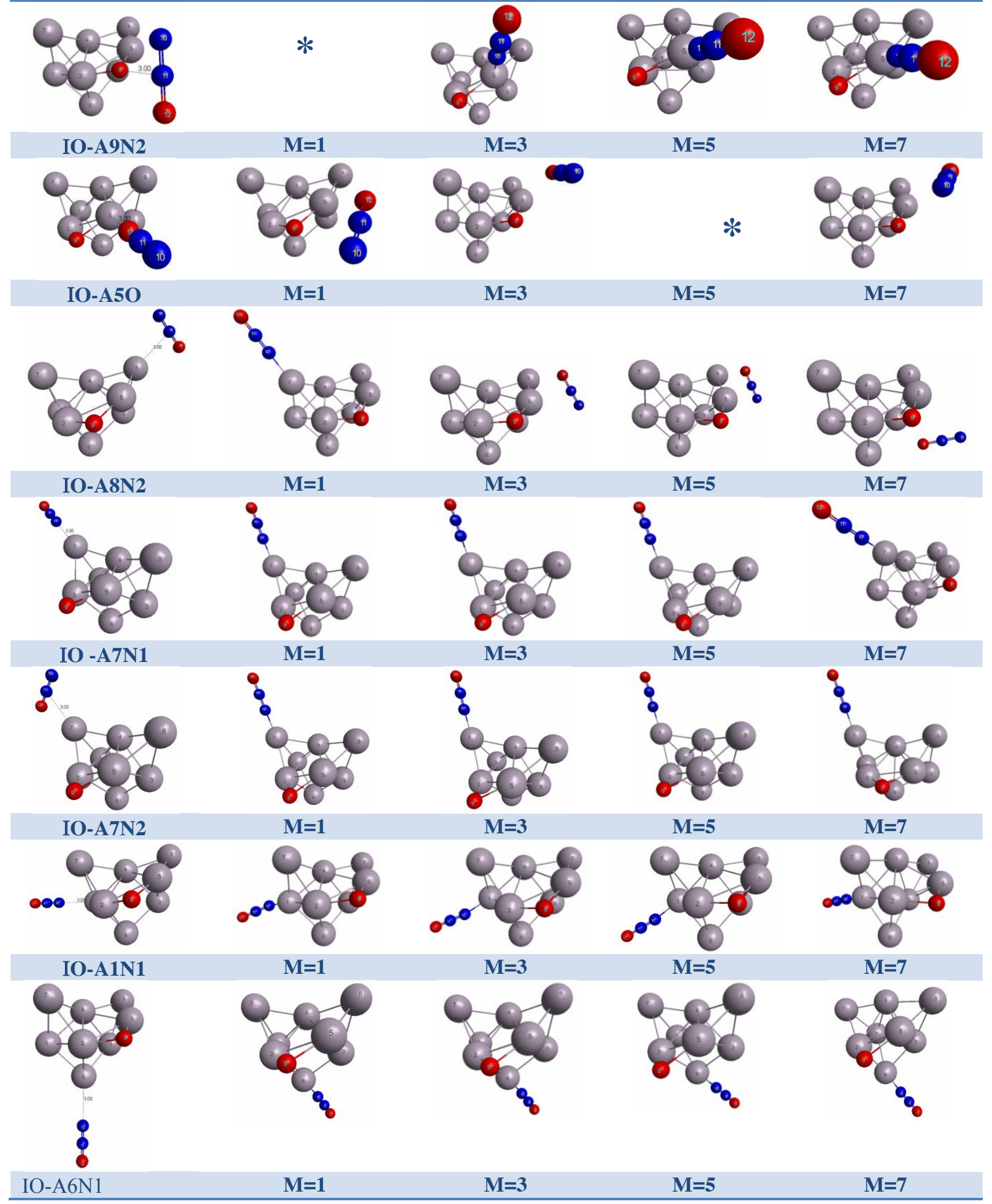


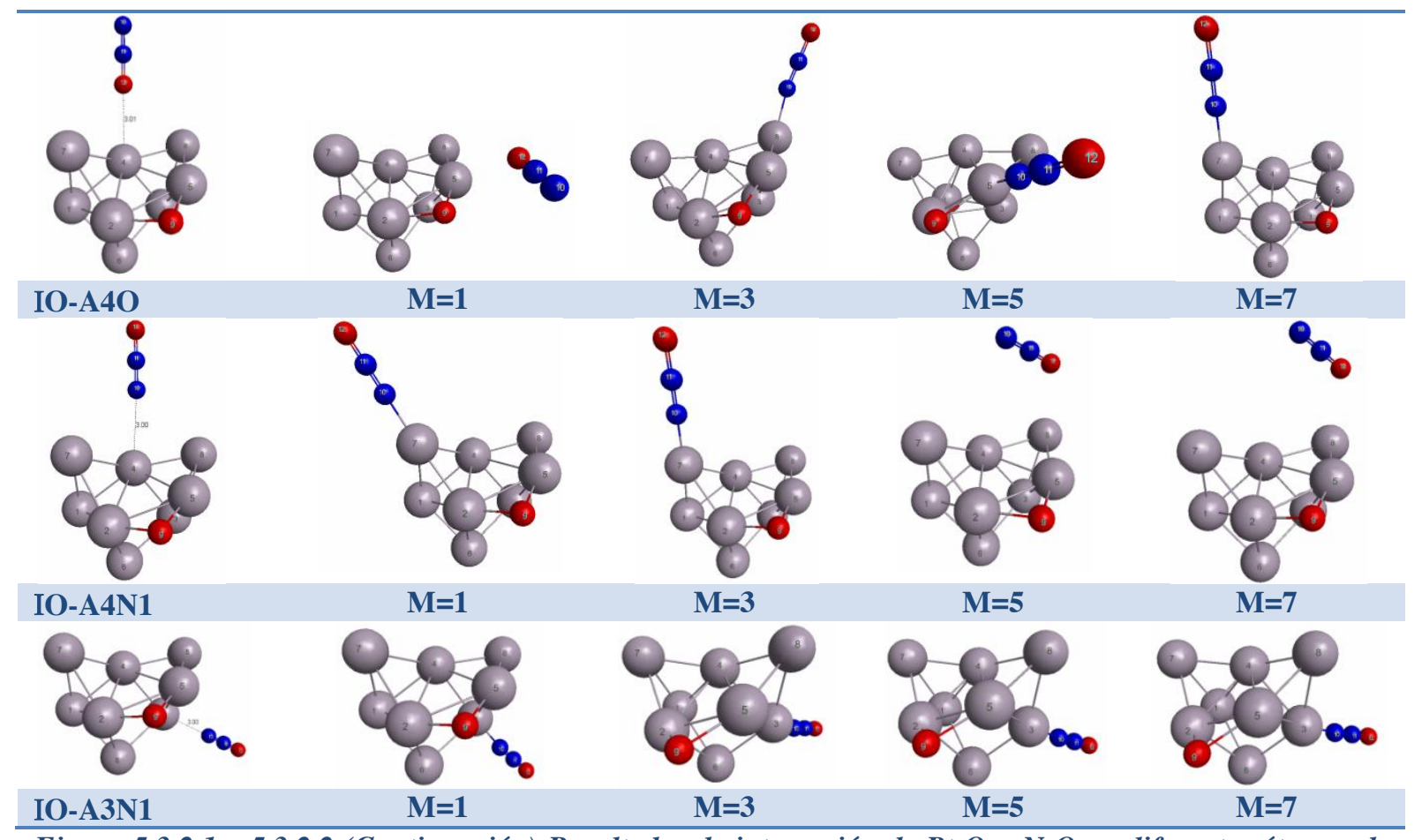

Figura 5.3.2.1 y 5.3.2.2 (Continuación) Resultados de interacción de $\mathrm{Pt}_{8} \mathrm{O}+\mathrm{N}_{2} \mathrm{O}$ en diferentes átomos de $P t$ por el átomo de $\mathrm{O}, \mathrm{N}$ intermedio y $\mathrm{N}$ terminal, con distintas multiplicidades de espín $(M=2 s+1)$. (*) Problemas de convergencia en el cálculo, no se obtienen resultados. 
Tabla 5.3.2.1 (Continuación) Parámetros energéticos de la interacción $\mathrm{Pt}_{8} \mathrm{O}+\mathrm{N}_{2} \mathrm{O}$ sobre átomos $\mathrm{Pt}$ por el átomo de $O$. Energías de disociación $\left(E_{\text {dis }}\right)$ y energía relativa $\left(E_{\text {relativa }}\right)$.

\begin{tabular}{|c|c|c|c|c|}
\hline $\begin{array}{c}\text { Tipo de } \\
\text { interacción }\end{array}$ & $\begin{array}{c}M \\
2 s+1\end{array}$ & $\begin{array}{c}\mathbf{E}_{\text {dis }} \\
\mathrm{kcal} / \mathrm{mol}\end{array}$ & $\begin{array}{c}\text { Erelativa }_{\text {kcal} / \mathbf{m o l}}\end{array}$ & $\begin{array}{c}\text { Enlace } \\
\text { /disociación }\end{array}$ \\
\hline \multirow{4}{*}{ IO-A9N2 } & 1 & --- & 53.12 & $*$ \\
\hline & 3 & -15.46 & 55.68 & $\mathrm{Si} / \mathrm{no}$ \\
\hline & 5 & -12.90 & 56.87 & $\mathrm{Si} / \mathrm{no}$ \\
\hline & 7 & -11.71 & & $\mathrm{Si} / \mathrm{no}$ \\
\hline \multirow{4}{*}{$\mathrm{IO}-\mathrm{A} 5 \mathrm{O}$} & 1 & -3.34 & 65.24 & $\mathrm{Si} / \mathrm{no}$ \\
\hline & 3 & -3.53 & 65.05 & $\mathrm{Si} / \mathrm{no}$ \\
\hline & 5 & --- & 76.28 & $*$ \\
\hline & 7 & 7.70 & & $\mathrm{Si} / \mathrm{no}$ \\
\hline \multirow{4}{*}{$\mathrm{IO}-8 \mathrm{~N} 2$} & 1 & -22.16 & 46.38 & $\mathrm{Si} / \mathrm{no}$ \\
\hline & 3 & -2.35 & 66.19 & $\mathrm{Si} / \mathrm{no}$ \\
\hline & 5 & 1.67 & 70.21 & $\mathrm{Si} / \mathrm{no}$ \\
\hline & 7 & 6.40 & 74.94 & $\mathrm{Si} / \mathrm{no}$ \\
\hline \multirow{4}{*}{ IO-A7N1 } & 1 & -22.16 & 46.38 & $\mathrm{Si} / \mathrm{no}$ \\
\hline & 3 & -20.15 & 46.39 & $\mathrm{Si} / \mathrm{no}$ \\
\hline & 5 & -18.66 & 49.88 & $\mathrm{Si} / \mathrm{no}$ \\
\hline & 7 & -7.31 & 61.23 & $\mathrm{Si} / \mathrm{no}$ \\
\hline \multirow{4}{*}{ IO-A7N2 } & 1 & -22.16 & 46.38 & $\mathrm{Si} / \mathrm{no}$ \\
\hline & 3 & -20.15 & 46.39 & $\mathrm{Si} / \mathrm{no}$ \\
\hline & 5 & -18.63 & 46.91 & $\mathrm{Si} / \mathrm{no}$ \\
\hline & 7 & -13.91 & 54.63 & si/no \\
\hline \multirow{4}{*}{ IO-A1N } & 1 & -11.61 & 56.93 & $\mathrm{Si} / \mathrm{no}$ \\
\hline & 3 & -10.98 & 57.56 & $\mathrm{Si} / \mathrm{no}$ \\
\hline & 5 & -7.34 & 61.20 & $\mathrm{Si} / \mathrm{no}$ \\
\hline & 7 & -7.75 & 60.79 & $\mathrm{Si} / \mathrm{no}$ \\
\hline \multirow{4}{*}{ IO-A6N1 } & 1 & -20.57 & 47.97 & $\mathrm{Si} / \mathrm{no}$ \\
\hline & 3 & -21.04 & 47.50 & $\mathrm{Si} / \mathrm{no}$ \\
\hline & 5 & -16.30 & 52.24 & $\mathrm{Si} / \mathrm{no}$ \\
\hline & 7 & -14.55 & 53.99 & $\mathrm{Si} / \mathrm{no}$ \\
\hline \multirow{4}{*}{$\mathrm{IO}-\mathrm{A} 4 \mathrm{O}$} & 1 & -3.33 & 65.21 & $\mathrm{Si} / \mathrm{no}$ \\
\hline & 3 & -8.75 & 59.79 & $\mathrm{Si} / \mathrm{no}$ \\
\hline & 5 & -12.90 & 55.64 & $\mathrm{Si} / \mathrm{no}$ \\
\hline & 7 & -9.94 & 58.60 & $\mathrm{Si} / \mathrm{no}$ \\
\hline \multirow{4}{*}{ IO-A4N1 } & 1 & -22.16 & 46.38 & $\mathrm{Si} / \mathrm{no}$ \\
\hline & 3 & -18.08 & 50.46 & $\mathrm{Si} / \mathrm{no}$ \\
\hline & 5 & 2.20 & 70.74 & $\mathrm{Si} / \mathrm{no}$ \\
\hline & 7 & 6.95 & 75.49 & $\mathrm{Si} / \mathrm{no}$ \\
\hline
\end{tabular}




\begin{tabular}{lllll}
\hline & 1 & -15.00 & 53.54 & $\mathrm{Si} /$ no \\
IO-A3N1 & 3 & -16.95 & 51.59 & $\mathrm{Si} /$ no \\
& 5 & -13.07 & 55.47 & $\mathrm{Si} /$ no \\
& 7 & -13.07 & 55.47 & $\mathrm{Si} /$ no \\
\hline \hline
\end{tabular}


Tabla 5.3.2.2 (Continuación) Parámetros geométricos de la interacción de $\mathrm{Pt}_{8} \mathrm{O}$ sobre átomos de Pt por el átomo de $\mathrm{N}$ terminal, por el átomo de $\mathrm{N}$ intermedio y por el átomo de $\mathrm{O}$. Multiplicidad (M), Frecuencias de vibración (v), distancias de enlace (d).

\begin{tabular}{|c|c|c|c|c|c|c|}
\hline $\begin{array}{c}\text { Tipo de } \\
\text { interacción }\end{array}$ & $\begin{array}{c}M \\
2 s+1\end{array}$ & $\begin{array}{l}v^{N-N} \\
\mathrm{~cm}^{-1}\end{array}$ & $\begin{array}{c}\mathbf{d P t}_{\text {Pto }} \\
\AA\end{array}$ & $\begin{array}{c}\mathbf{d}_{\mathrm{Pt}-\mathrm{N}} \\
\AA\end{array}$ & $\begin{array}{c}\mathbf{d}_{\mathbf{P t}-\mathrm{Pt}} \\
\AA\end{array}$ & $\begin{array}{c} \pm \mathbf{d}_{\text {Pt-Pt }} \\
\AA\end{array}$ \\
\hline & 1 & 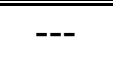 & ב--- & ב--- & ב--- & * \\
\hline \multirow[t]{4}{*}{ IO-A9N2 } & 3 & 1256 & - & 1.97 & 2.65 & 0.12 \\
\hline & 5 & 1258 & - & 1.97 & 2.66 & 011 \\
\hline & 7 & 1259 & - & 1.97 & 2.66 & 0.11 \\
\hline & 1 & 2267 & 2.48 & - & 2.61 & 0.09 \\
\hline \multirow[t]{4}{*}{ IO-A5O } & 3 & 2265 & 2.39 & - & 2.62 & 0.08 \\
\hline & 5 & --- & --- & --- & --- & $*$ \\
\hline & 7 & 2281 & 3.33 & - & 2.62 & 0.06 \\
\hline & 1 & 1249 & - & 1.94 & 2.63 & 0.09 \\
\hline \multirow[t]{4}{*}{$\mathrm{IO}-8 \mathrm{~N} 2$} & 3 & 2268 & 2.55 & - & 2.62 & 0.08 \\
\hline & 5 & 2267 & 2.53 & - & 2.61 & 0.08 \\
\hline & 7 & 2269 & 2.54 & - & 2.62 & 0.10 \\
\hline & 1 & 1249 & - & 1.94 & 2.63 & 0.09 \\
\hline \multirow[t]{4}{*}{ IO-A7N1 } & 3 & 1252 & - & 1.93 & 2.63 & 0.09 \\
\hline & 5 & 1257 & - & 1.93 & 2.62 & 0.08 \\
\hline & 7 & 1251 & - & 1.96 & 2.63 & 0.09 \\
\hline & 1 & 1249 & - & 1.94 & 2.63 & 0.09 \\
\hline \multirow[t]{4}{*}{$\mathrm{IO}-\mathrm{A} 7 \mathrm{~N} 2$} & 3 & 1253 & - & 1.93 & 2.63 & 0.09 \\
\hline & 5 & 1256 & - & 1.93 & 2.63 & 0.10 \\
\hline & 7 & 1248 & - & 1.94 & 2.62 & 0.08 \\
\hline & 1 & 1242 & - & 2.04 & 2.62 & 0.08 \\
\hline \multirow[t]{4}{*}{$\mathrm{IO}-\mathrm{A} 1 \mathrm{~N}$} & 3 & 1251 & - & 2.00 & 2.63 & 0.09 \\
\hline & 5 & 1253 & - & 1.97 & 2.63 & 0.09 \\
\hline & 7 & 1245 & - & 1.98 & 2.65 & 012 \\
\hline & 1 & 1252 & - & 1.94 & 2.63 & 0.09 \\
\hline \multirow[t]{4}{*}{ IO-A6N1 } & 3 & 1254 & - & 1.93 & 2.63 & 0.09 \\
\hline & 5 & 1255 & - & 1.93 & 2.62 & 0.08 \\
\hline & 7 & 1253 & - & 1.93 & 2.65 & 012 \\
\hline & 1 & 2265 & 2.48 & - & 2.62 & 0.08 \\
\hline \multirow[t]{5}{*}{ IO-A4O } & 3 & 1247 & - & 2.01 & 2.65 & 0.12 \\
\hline & 5 & 1258 & - & 1.97 & 2.66 & 0.11 \\
\hline & 7 & 1256 & - & 1.95 & 2.63 & 0.09 \\
\hline & 1 & 1249 & - & 1.94 & 2.63 & 0.09 \\
\hline & 3 & 1253 & - & 1.95 & 2.62 & 0.08 \\
\hline
\end{tabular}




\begin{tabular}{lllcccc} 
IO-A4N1 & 5 & 2272 & 2.78 & - & 2.61 & 0.08 \\
& 7 & 2273 & 2.72 & - & 2.62 & 0.09 \\
\hline \multirow{3}{*}{ IO-A3N1 } & 1 & 1245 & - & 1.97 & 2.63 & 0.09 \\
& 3 & 1255 & - & 1.97 & 2.62 & 0.08 \\
& 5 & 1252 & - & 1.95 & 2.62 & 0.08 \\
& 7 & 1252 & - & 1.95 & 2.62 & 0.12 \\
\hline \hline
\end{tabular}


II. Interacción a enlaces.

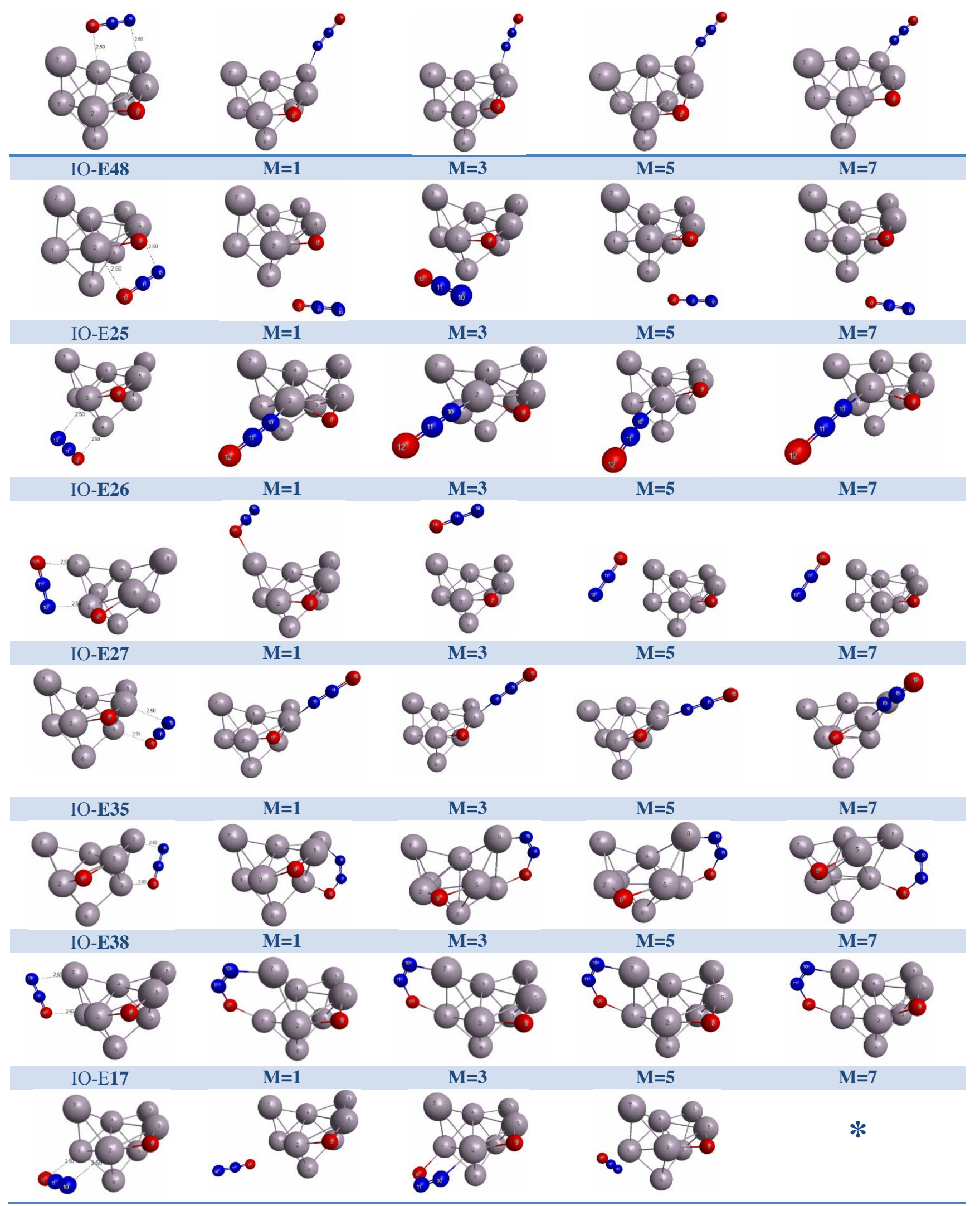




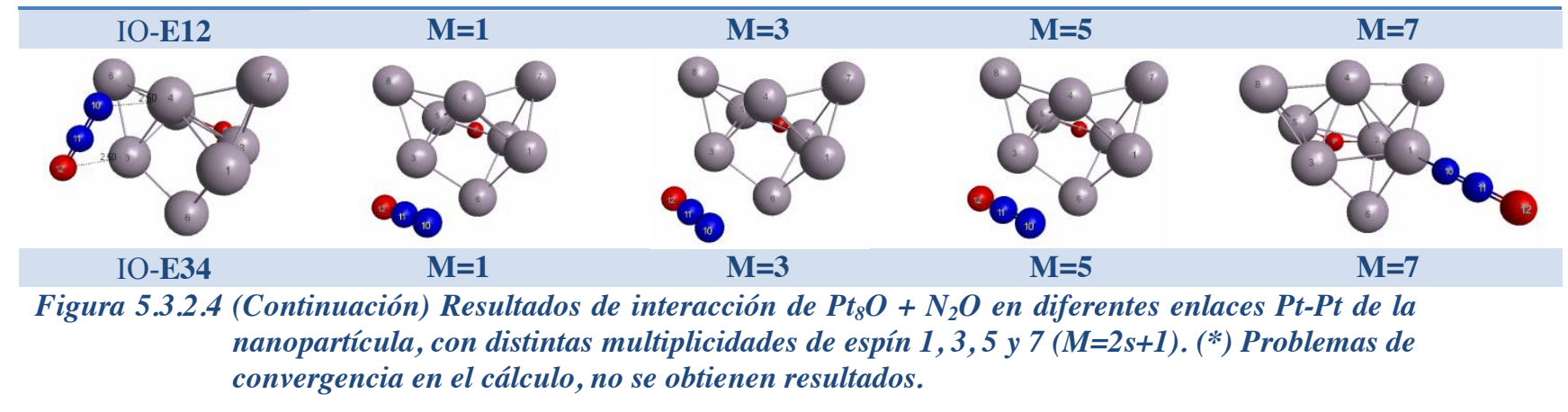


Tabla 5.3.2.5 (Continuación) Parámetros energéticos para la interacción $\mathrm{Pt}_{8} \mathrm{O}+\mathrm{N}_{2} \mathrm{O}$ por enlaces Pt-Pt. Energías de disociación $\left(E_{\text {dis }}\right)$ y cambios de energía relativa $\left(E_{\text {relativa }}\right)$.

\begin{tabular}{|c|c|c|c|c|}
\hline $\begin{array}{c}\text { Tipo de } \\
\text { interacción }\end{array}$ & $\begin{array}{c}M \\
2 s+1\end{array}$ & $\begin{array}{c}\mathbf{E}_{\mathrm{dis}} \\
\mathrm{kcal} / \mathrm{mol}\end{array}$ & $\begin{array}{c}\mathrm{E}_{\text {relativa }} \\
\mathrm{kcal} / \mathrm{mol}\end{array}$ & $\begin{array}{c}\text { Enlace/ } \\
\text { disociación }\end{array}$ \\
\hline \multirow{4}{*}{ IO-E48 } & $\overline{1} 1$ & -8.80 & 259.74 & $\overline{\mathrm{Si}} / \mathrm{no}$ \\
\hline & 3 & -8.75 & 59.79 & $\mathrm{Si} / \mathrm{no}$ \\
\hline & 5 & -8.71 & 59.83 & $\mathrm{Si} / \mathrm{no}$ \\
\hline & 7 & -6.50 & 62.04 & $\mathrm{Si} /$ no \\
\hline \multirow{4}{*}{ IO-E25 } & 1 & -2.01 & 66.53 & $\mathrm{Si} / \mathrm{no}$ \\
\hline & 3 & -1.15 & 67.39 & $\mathrm{Si} / \mathrm{no}$ \\
\hline & 5 & 1.27 & 69.81 & $\mathrm{Si} / \mathrm{no}$ \\
\hline & 7 & 6.05 & 74.59 & $\mathrm{Si} /$ no \\
\hline \multirow{4}{*}{ IO-E26 } & 1 & -14.81 & 53.73 & $\mathrm{Si} / \mathrm{no}$ \\
\hline & 3 & -17.88 & 50.66 & $\mathrm{Si} / \mathrm{no}$ \\
\hline & 5 & -2.48 & 66.06 & $\mathrm{Si} /$ no \\
\hline & 7 & -15.26 & 53.28 & $\mathrm{Si} / \mathrm{no}$ \\
\hline \multirow{4}{*}{ IO-E27 } & 1 & -22.15 & 46.39 & $\mathrm{Si} / \mathrm{no}$ \\
\hline & 3 & -17.29 & 51.25 & $\mathrm{Si} /$ no \\
\hline & 5 & -13.82 & 54.72 & $\mathrm{Si} / \mathrm{no}$ \\
\hline & 7 & -7.31 & 61.23 & $\mathrm{Si} / \mathrm{no}$ \\
\hline \multirow{4}{*}{ IO-B35 } & 1 & -14.15 & 54.39 & $\mathrm{Si} / \mathrm{no}$ \\
\hline & 3 & -15.47 & 53.07 & $\mathrm{Si} / \mathrm{no}$ \\
\hline & 5 & -12.90 & 55.64 & $\mathrm{Si} / \mathrm{no}$ \\
\hline & 7 & -11.71 & 56.83 & $\mathrm{Si} / \mathrm{no}$ \\
\hline \multirow{4}{*}{ IO-E38 } & 1 & -9.85 & 58.69 & $\mathrm{Si} / \mathrm{no}$ \\
\hline & 3 & -17.70 & 50.84 & $\mathrm{Si} / \mathrm{no}$ \\
\hline & 5 & -13.53 & 55.01 & $\mathrm{Si} / \mathrm{no}$ \\
\hline & 7 & -2.56 & 65.98 & $\mathrm{Si} / \mathrm{no}$ \\
\hline \multirow{4}{*}{ IO-E17 } & 1 & -18.17 & 50.37 & $\mathrm{Si} / \mathrm{no}$ \\
\hline & 3 & -19.02 & 49.52 & $\mathrm{Si} / \mathrm{no}$ \\
\hline & 5 & -10.64 & 57.90 & $\mathrm{Si} / \mathrm{no}$ \\
\hline & 7 & -6.71 & 61.83 & $\mathrm{Si} / \mathrm{no}$ \\
\hline \multirow{4}{*}{ IO-E12 } & 1 & -1.96 & 66.58 & $\mathrm{Si} / \mathrm{no}$ \\
\hline & 3 & -4.27 & 64.27 & $\mathrm{Si} / \mathrm{no}$ \\
\hline & 5 & --- & --- & $*$ \\
\hline & 7 & --- & --- & $*$ \\
\hline
\end{tabular}


Tabla 5.3.2.6 (Continuación) Parámetros geométricos de la interacción $\mathrm{Pt}_{8} \mathrm{O}+\mathrm{N}_{2} \mathrm{O}$ por enlaces Pt-Pt.Multiplicidad (M), Frecuencia de vibración (v), distancias de enlace (d).

\begin{tabular}{|c|c|c|c|c|c|c|}
\hline $\begin{array}{c}\text { Tipo de } \\
\text { interacción }\end{array}$ & $\begin{array}{c}M \\
2 s+1\end{array}$ & $\begin{array}{l}v^{N-N} \\
\mathbf{c m}^{-1}\end{array}$ & $\underset{\AA}{\stackrel{\mathbf{d}_{\text {Pt-O }}}{\AA}}$ & $\underset{\AA}{\mathbf{d}_{\mathrm{Pt}-\mathrm{N}}}$ & $\begin{array}{c}\mathbf{d}_{\mathbf{P t}-\mathbf{P t}} \\
\AA\end{array}$ & $\underset{\AA}{ \pm \underset{\mathbf{A} t-P t}{ }}$ \\
\hline \multirow{4}{*}{ IO-E48 } & 1 & 11240 & - & 2.04 & 2.61 & 0.08 \\
\hline & 3 & 1247 & - & 2.01 & 2.65 & 0.12 \\
\hline & 5 & 1249 & - & 2.01 & 2.67 & 0.14 \\
\hline & 7 & 1249 & - & 2.01 & 2.67 & 0.14 \\
\hline \multirow{4}{*}{ IO-E25 } & 1 & 2262 & 2.54 & - & 2.62 & 0.08 \\
\hline & 3 & 2280 & 3.14 & - & 2.61 & 0.08 \\
\hline & 5 & 2259 & 2.39 & - & 2.61 & 0.09 \\
\hline & 7 & 2262 & 2.41 & - & 2.62 & 0.08 \\
\hline \multirow{4}{*}{ IO-E26 } & 1 & 1268 & - & 2.00 & 2.60 & 0.09 \\
\hline & 3 & 1268 & - & 1.97 & 2.60 & 0.09 \\
\hline & 5 & 1268 & - & 2.00 & 2.62 & 0.08 \\
\hline & 7 & 1270 & - & 1.97 & 2.63 & 0.09 \\
\hline \multirow{4}{*}{ IO-E27 } & 1 & 1248 & - & 1.94 & 2.63 & 0.08 \\
\hline & 3 & 1245 & - & 1.95 & 2.66 & 0.08 \\
\hline & 5 & 1251 & - & 1.95 & 2.63 & 0.10 \\
\hline & 7 & 1250 & - & 1.96 & 2.63 & 0.08 \\
\hline \multirow{4}{*}{ IO-B35 } & 1 & 1255 & - & 1.98 & 2.63 & 0.09 \\
\hline & 3 & 1260 & - & 1.97 & 2.65 & 011 \\
\hline & 5 & 1258 & - & 1.97 & 2.66 & 0.09 \\
\hline & 7 & 1259 & - & 1.97 & 2.66 & 0.09 \\
\hline \multirow{4}{*}{ IO-E38 } & 1 & 1616 & 2.13 & 2.00 & 2.62 & 0.08 \\
\hline & 3 & 1472 & 2.00 & 1.90 & 2.53 & 0.14 \\
\hline & 5 & 1474 & 2.01 & 1.90 & 2.67 & 0.14 \\
\hline & 7 & 1572 & 2.10 & 1.98 & 2.53 & 0.12 \\
\hline \multirow{4}{*}{ IO-E17 } & 1 & 1465 & 2.04 & 1.91 & 2.61 & 0.08 \\
\hline & 3 & 1470 & 2.02 & 1.91 & 2.61 & 0.08 \\
\hline & 5 & 1499 & 2.06 & 1.94 & 2.63 & 0.09 \\
\hline & 7 & 1456 & 2.04 & 1.93 & 2.64 & 012 \\
\hline \multirow{4}{*}{ IO-E12 } & 1 & 2269 & 2.69 & - & 2.62 & 0.08 \\
\hline & 3 & 1601 & 2.10 & 2.03 & 2.63 & 0.09 \\
\hline & 5 & 2271 & 2.64 & - & 2.62 & 0.08 \\
\hline & 7 & --- & --- & --- & --- & $*$ \\
\hline
\end{tabular}




\title{
CONSTANCIA DE PRESENTACION DE EXAMEN DE GRADO
}

La Universidad Autónoma Metropolitana extiende la presente CONSTANCIA DE PRESENTACION DE EXAMEN DE GRADO de MAESTRA EN CIENCIAS (QUIMICA) de la alumna ERENDIDA HERNANDEZ VERA, matrícula 2123803290, quien cumplió con los 148 créditos correspondientes a las unidades de enseñanza aprendizaje del plan de estudio. Con fecha veintiuno de noviembre del 2014 presentó la DEFENSA de su EXAMEN DE GRADO cuya denominación es:

\author{
ESTUDIO TEORICO DE LA \\ REDUCCCION DE N $\mathrm{N}_{2} \mathrm{O}$ SOBRE \\ NANOPARTICULAS DE $\mathrm{Pt}_{8}$ Y \\ $\mathrm{Pt}_{8} \mathrm{O}$, EMPLEANDO TEORIA DE \\ FUNCIONALES DE LA \\ DENSIDAD (DFT).
} Cabe mencionar que la aprobación del Examen de Grado tiene un valor de 60
créditos y el programa consta de 183 créditos.

El jurado del examen ha tenido a bien otorgarle la calificación de:

$$
\text { APROBAR }
$$

\section{Presidente}

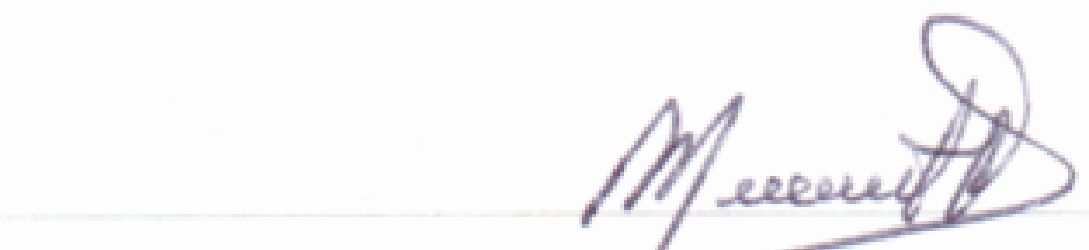

DR. FRANCISCO MIGUEL CASTRO MARTINEZ
JURADO

Secretario

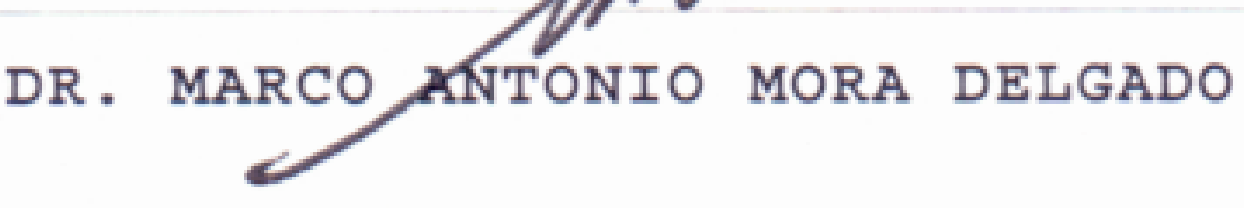

Vocal

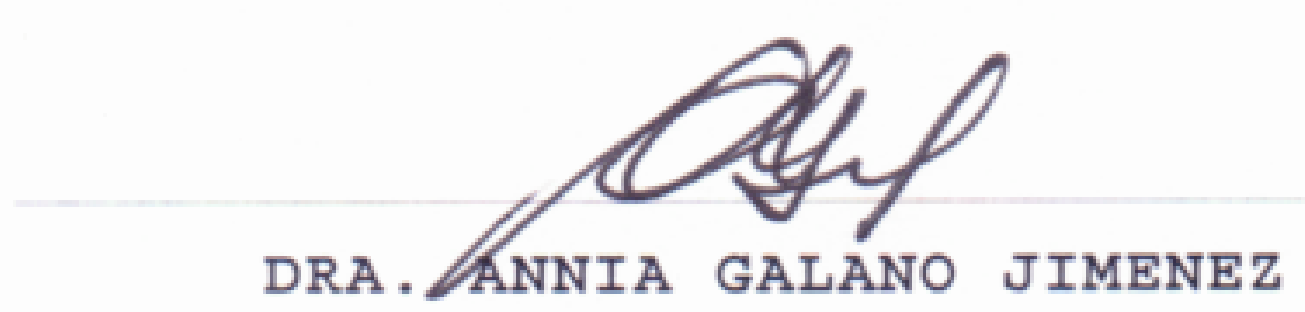

Coordinación de Sistemas Escolares

Av. San Rafael Atlixco 186. Col. Vicentina, México, D.F. C.P. 09340 Tels. 5804-4880 y 5804-4883 csera@xanum.uam.mx www.izt.uam.mx 